\title{
Unsymmetrical Iron P-NH-P' Catalysts for the Asymmetric Pressure Hydrogenation of Aryl Ketones
}

Samantha A. M. Smith Paraskevi O. Lagaditis, Anne Lüpke, Alan J, Lough, Robert H. Morris

Version Post-print/accepted manuscript

Citation S. A. M. Smith, P. O. Lagaditis, A. Lupke, A. J. Lough and R. H.

(published version) Morris, Chem. - Eur. J., 2017, 23, 7212-7216.

http://pubs.acs.org/doi/10.1002/chem.201701254

\section{Copyright / License}

\author{
Publisher's Statement This document is the Accepted Manuscript version of a Published Work \\ that appeared in final form in Chem. Eur. J., copyright @ W Wiley-VCH \\ after peer review and technical editing by the publisher. To access the \\ final edited and published work see \\ http://pubs.acs.org/doi/10.1002/chem.201701254
}

How to cite TSpace items

Always cite the published version, so the author(s) will receive recognition through services that track citation counts, e.g. Scopus. If you need to cite the page number of the author manuscript from TSpace because you cannot access the published version, then cite the TSpace version in addition to the published version using the permanent URI (handle) found on the record page.

This article was made openly accessible by $U$ of $T$ Faculty. Please tell us how this access benefits you. Your story matters. 


\title{
Unsymmetrical Iron P-NH-P' Catalysts for the Asymmetric Pressure Hydrogenation of Aryl Ketones
}

\author{
Samantha A. M. Smith ${ }^{[+][a]}$ Paraskevi O. Lagaditis ${ }^{[+][a]}$, Anne Lüpke ${ }^{[b]}$, Alan J, Lough ${ }^{[a]}$, Robert H. \\ Morris*[a] \\ Celebrating 100 years of CSC conferences in Canada and 150 years of the GDCh in Germany.
}

\begin{abstract}
The reductive amination of $\alpha$-dialkylphosphine acetaldehydes with enantiopure $\beta$-aminophosphines is a new, versatile route to unsymmetrical tridentate (pincer) ligands $\mathrm{P}-\mathrm{NH}-\mathrm{P}$ '. Four new ligands $\mathrm{PR}_{2} \mathrm{CH}_{2} \mathrm{CH}_{2} \mathrm{NHCHR}^{\prime} \mathrm{CHR} \mathrm{PPh}_{2}\left(\mathrm{R}=\mathrm{iPr}, \mathrm{Cy}, \mathrm{R}^{\prime}=\right.$ $\left.\mathrm{Ph}, \mathrm{CH}\left(\mathrm{CH}_{3}\right)_{2}, \mathrm{R}^{\prime \prime}=\mathrm{Ph}, \mathrm{H}\right)$ prepared in this way are used to make the iron(II) complexes mer- $\mathrm{FeCl}_{2}(\mathrm{CO})\left(\mathrm{P}-\mathrm{NH}-\mathrm{P}^{\prime}\right)$ and $m e r-\mathrm{FeCl}(\mathrm{H})(\mathrm{CO})(\mathrm{P}$ $\left.\mathrm{NH}-\mathrm{P}^{\prime}\right)$. The hydride complex with the rigid ligand with $\mathrm{R}^{\prime}=\mathrm{R}^{\prime \prime}=\mathrm{Ph}$ is an efficient and highly enantioselective homogeneous asymmetric pressure hydrogenation (APH) catalyst. Prochiral aryl ketones are reduced under mild conditions (THF, $0.1 \mathrm{~mol} \%$ catalyst, $1 \mathrm{~mol} \%$ KOtBu, $5-10$ bar, $50^{\circ} \mathrm{C}$ ) to the (S)-alcohols, usually in enantiomeric excess (e.e.) greater than $90 \%$. DFT calculations provide transition state structures for the enantiodetermining hydride transfer step.
\end{abstract}

The homogeneous asymmetric pressure hydrogenation of prochiral ketones provides an efficient route to valuable non-racemic alcohols. ${ }^{[1]}$ While the best catalysts are based on ruthenium, osmium and iridium, ${ }^{[2]}$ more sustainable iron-based systems show much promise. ${ }^{[3]}$ The most enantioselective reductions to date (over $95 \%$ e.e. in the alcohol product) were reported by the Gao group who used a mixture of $\mathrm{Fe}_{3}(\mathrm{CO})_{12}$ and an enantiopure 22-membered -PNNPNNmacrocycle to catalyze the complete reduction of several aryl ketones. ${ }^{[4]}$ Our group reported the first well defined $\mathrm{Fe}$ (II) precatalysts for the APH of ketones, trans-[Fe(L)(CO)(PNNP) $]^{\text {nt }}$ containing enantiopure PNNP ligands but the e.e. and activity were low to moderate; ${ }^{[5]}$ such complexes are excellent for the asymmetric transfer hydrogenation (ATH) of ketones ${ }^{[6]}$ but cause a reduction in e.e. of the product alcohol at reaching the conversion determined by the equilibrium. An advantage of the irreversibility of the APH process is that complete conversion can be obtained with no degradation in the e.e. of the alcohol. Iron complexes with functionalized cyclopentadienyl and chiral ligands have so far displayed low activity

[a] S. A. M. Smith ${ }^{[+]}$, Dr. P. O. Lagaditis ${ }^{[+]}$, Dr. A. J. Lough, Prof. R. H. Morris

Department of Chemistry

University of Toronto

80 Saint George St., Toronto, Ontario, Canada M5S3H6

E-mail: rmorris@chem.utoronto.ca

[b] A. Lüpke

Department of Chemistry

Johannes Gutenberg University, Mainz

Saarstraße 21, 55122, Mainz, Germany

[+] These authors contributed equally.

Supporting information for this article is given via a link at the end of the document. CCDC 1525651 (S-7), 1538240 (S-8) and 1525652

(S-10) contain the supplementary crystallographic data for this

paper. These data can be obtained free of charge from The

Cambridge Crystallographic Data Centre via

www.ccdc.cam.ac.uk/data request/cif. and selectivity for the $\mathrm{APH}$ of ketones ${ }^{[7]}$ but more promising results for the $\mathrm{APH}$ of imines. ${ }^{\left[{ }^{[8]}\right.}$

Pincer iron complexes $\mathrm{Fe}(\mathrm{CO}) \mathrm{X}(\mathrm{Y})(\mathrm{PNP})$ (A, Figure 1), $\mathrm{X}, \mathrm{Y}$ $=$ halide, hydride, borohydride, PNP achiral ligand with trialkylphosphine and pyridyl donors, have excellent activity for the pressure hydrogenation of ketones as discovered by Milstein and coworkers. ${ }^{[9]}$ Similar complexes with achiral PNP ligands with trialkylphosphine and secondary amine donors (B) were shown by several groups to efficiently catalyze other hydrogenation and dehydrogenation applications. ${ }^{[10]}$

Our group reported the first such pincer complex (C) with an enantiopure ligand as a precursor for the APH of ketones to alcohols with e.e. up to $89 \%$ and an imine to amine in $90 \%$ e.e. ${ }^{[11]}$ The mixed phosphine imine-type PNP' ligand of $\mathbf{C}$, which forms in an iron-templated reaction, requires $\mathrm{LiAlH}_{4}$ reduction while on the metal to produce the $\mathrm{N}-\mathrm{H}$ functionality required for ketone activation and enantioselectivity. ${ }^{[12]}$ Some decomposition products were identified in this reaction including undesired $\mathrm{Fe}(\mathrm{CO})_{2}(\mathrm{PNP})$ complexes as a consequence of the dicarbonyl precursor structure. $[11,13]$ Thus a monocarbonyl precursor complex was targeted. We also found that ligands forming five membered rings -Fe-NH-C-C-PR $\mathrm{R}_{2}$ form more active complexes than those that form 6-membered rings and that $R=$ alkyl may provide more active catalysts than $\mathrm{R}=$ aryl. ${ }^{[13]}$ Zirakzadeh et al. have recently described the synthesis of similar complexes with enantiopure ferrocenylphosphine ligands; e.e. up to $81 \%$ were observed for the APH of aryl ketones. ${ }^{[14]}$ Kirchner's group has also prepared chiral iron complexes with unsymmetrical PNP' ligands for the hydrogenation of ketones but no e.e. was reported. ${ }^{[15]}$ Based on these developments, we targeted the synthesis of a new family of chiral pincer ligands and their iron APH catalysts as described here.

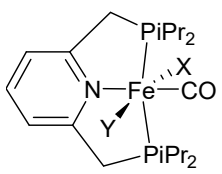

A

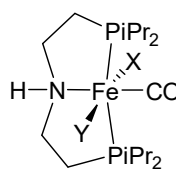

B

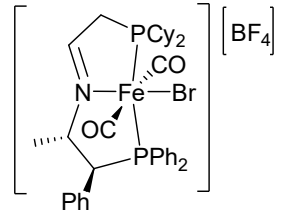

C
$\mathrm{X}, \mathrm{Y}=\mathrm{H}, \mathrm{BH}_{4}$, halide

Figure 1. Iron hydrogenation and dehydrogenation catalysts with pincer ligands.

Reductive amination has proven to be a successful method for the synthesis of the new ligands 1 to 4 (Scheme 1). 


$$
\begin{aligned}
& \text { 等 } \\
& \text { 1: R=iPr, R', R"=Ph } \\
& \text { 4: } \mathrm{R}=\mathrm{Cy}, \mathrm{R}^{\prime}, \mathrm{R}^{\prime}=\mathrm{Ph}
\end{aligned}
$$

Scheme 1. Synthesis of new chiral P-NH-P' ligands.

Two equivalents of the hydride reagent are added, the first to release the $\alpha$-diisopropylphosphine acetaldehyde by deprotonation of the phosphonium dimer, ${ }^{[16]}$ and the second to reduce the imine formed by the condensation of this aldehyde with the primary amino group of the $\beta$-aminophosphine. Normally this method would yield tertiary amine products ${ }^{[17]}$ however we find that the substituent $\alpha$ to the amine of the starting material lends enough steric bulk to protect the secondary amine from further reaction. Due to the availability of a range of enantiopure $\beta$-aminophosphines ${ }^{[18]}$ and our air- and moisture-stable phosphonium dimers, ${ }^{[19]}$ a large variety of these ligands can be synthesized in this manner. The characterization of ligands 1-4 is discussed in the Supporting Information.

Ligands 1-4 were stirred with anhydrous iron dichloride in tetrahydrofuran (THF) under $\mathrm{CO}$ gas to yield the complexes $(\mathbf{S}, \mathbf{S})-\mathbf{5}$, $(S, S)-6,(S)-7$, and $(S)-8$ in high yields as purple solids (Scheme 2). They are synthesized as a single trans-dichloro isomer as signalled by a characteristic set of doublets with a large ${ }^{2} J_{\mathrm{PP}}$ coupling constant $(177 \mathrm{~Hz})$ in the ${ }^{31} \mathrm{P}\left\{{ }^{1} \mathrm{H}\right\}$ NMR spectra. The crystal structures of $(\mathbf{S})-7$ (Figure 2) and (S)-8 (Supporting Information) shows that they have the expected mer configuration with the $\mathrm{C}-\mathrm{H}$ on the chiral carbon anti with respect to the $\mathrm{N}-\mathrm{H}$ group. A very similar structure with the symmetrical $\left(\mathrm{PiPr}_{2} \mathrm{CH}_{2} \mathrm{CH}_{2}\right)_{2} \mathrm{NH}$ ligand has been reported. ${ }^{[20]}$

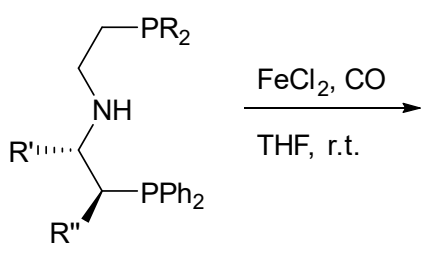

$(S, S)-5: R=i P r, R^{\prime}, R^{\prime \prime}=P h$ (S, S)-6: R=Cy, R', R"=Ph (S)-7: R=iPr, R'=Ph R"=H (S)-8: R=iPr, R'=iPr R"=H

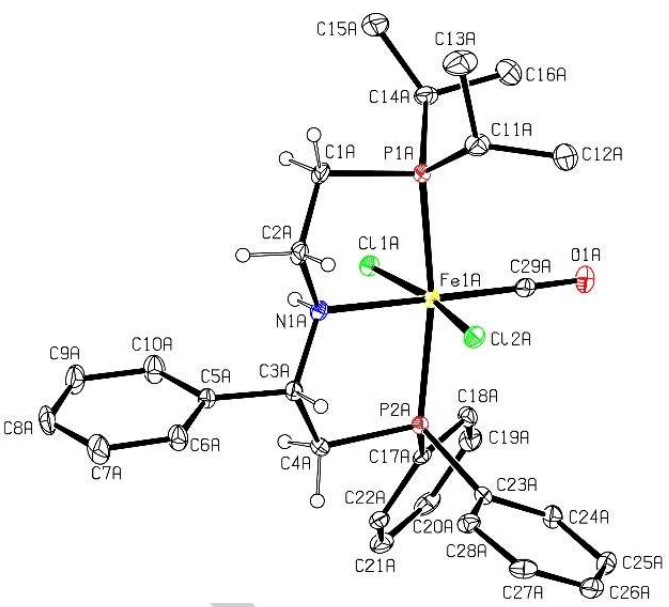

Figure 2. Structure of $(S)-7$ (molecule A) with thermal ellipsoids set at $30 \%$ probability. Selected bond lengths $[\AA]]$ and angles $\left[{ }^{\circ}\right]$ : Fe1-P1 2.279(1), Fe1-P2 2.254(1), Fe1-Cl1 2.329(1), Fe1-Cl2 2.316(1), Fe1-C29 1.757(5), Fe1-N1 2.081(4), N1-H 0.86(4), C29-O1 1.129(5); N1-Fe1-P1 85.2(1), N1-Fe1-P2 84.7(1), P2-Fe1-P1 169.99(5), Cl2-Fe1-Cl1 171.02(5), C29-Fe1-N1 177.8(2).

The monohydride complexes are prepared by treating the dichloro complexes with Superhydride $\left(\mathrm{LiHBEt}_{3}\right)$ at $-30^{\circ}$ (Scheme 3). (S,S)-9 exists as predominantly $(87 \%)$ one isomer in solution, with characteristic ${ }^{31} \mathrm{P}\left\{{ }^{1} \mathrm{H}\right\}$ spectra displaying two doublets with a large coupling constant ${ }^{2} J_{\mathrm{PP}}(138 \mathrm{~Hz})$, again suggesting a mer configuration of the complex. The major isomer has an associated doublet of doublet pattern in the hydride region of the ${ }^{1} \mathrm{H}$ NMR spectrum with a chemical shift of $-21.5 \mathrm{ppm}$. Minor amounts of two other isomers are present (see SI). Complex (S,S)-6 was reacted with Superhydride, however the reaction produced a mixture of free ligand and several unidentified phosphorus-containing species. The spectra of (S)-10 are complex because of the presence of four isomers with doublet of doublet or triplet hydride resonances at $-20.47 \mathrm{ppm}(5 \%),-20.96$ (22\%), $-21.40(31 \%)$ and $-22.05 \mathrm{ppm}(42 \%)$. Clearly the extra phenyl group in (S,S)-9 is needed to restrict the number of diastereomers. One of the isomers of $(S)-10$ has been crystallographically characterized as shown in Figure 3. The $\mathrm{N}-\mathrm{H}$ and $\mathrm{Fe}-\mathrm{H}$ bonds are almost parallel with a dihedral angle $\mathrm{H}-\mathrm{N} 1-\mathrm{Fe} 1-\mathrm{H}$ of $7.0^{\circ}$. In the crystal structure with the symmetrical $\left(\mathrm{PiPr}_{2} \mathrm{CH}_{2} \mathrm{CH}_{2}\right)_{2} \mathrm{NH}$ ligand, the $\mathrm{N}-\mathrm{H}$ is found instead parallel to the $\mathrm{Fe}-\mathrm{Cl}$ unit. ${ }^{[20]}$ The synthesis of (S)-11 was not selective with a mixture of up to six hydride complexes shown in the ${ }^{1} \mathrm{H}$ NMR spectrum. This complex was not tested due to the complicated mixture of isomers.

Scheme 2. Synthesis of $\mathrm{Fe}(\mathrm{Cl})_{2}(\mathrm{CO})\left(\mathrm{P}-\mathrm{NH}-\mathrm{P}^{\prime}\right)$ complexes. 


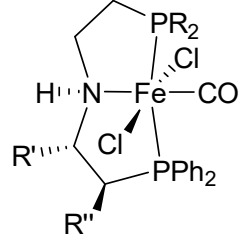

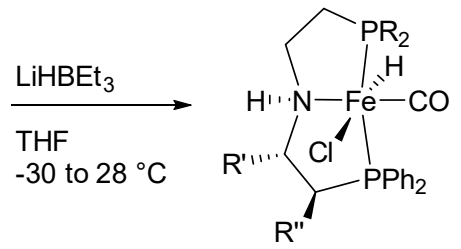

(S,S)-9: $R=i P r, R^{\prime}=R^{\prime \prime}=P h$

(S)-10: $\mathrm{R}=\mathrm{iPr}, \mathrm{R}^{\prime}=\mathrm{Ph}, \mathrm{R}^{\prime \prime}=\mathrm{H}$

(S)-11: $R=\mathrm{R}^{\prime}=\mathrm{iPr}, \mathrm{R}^{\prime \prime}=\mathrm{H}$

Scheme 3. Synthesis of iron chloro(hydrido) complexes.

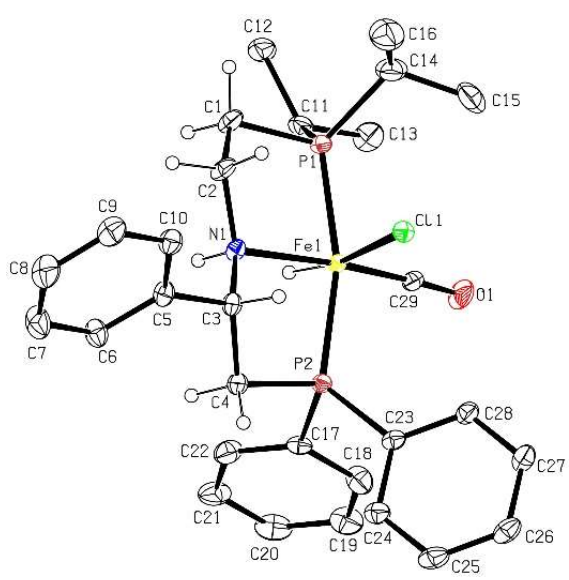

Figure 3 Crystal structure of $(S)-10$. Selected bond lengths $[\AA]$ and angles $\left[^{\circ}\right]$ : Fe1-H 1.40(4), Fe1-C29 1.717(4), Fe1-N1 2.093(3), Fe1-P2 2.189(1), Fe1-P1 2.218(1), Fe1-Cl1 2.4116(1), O1-C29 1.164(5), N1-H 0.90(6); N1-Fe1-P1 85.8(1), N1-Fe1-P2 83.7(1), H-Fe1-N1 84.1(16), C29-Fe1-N1 171.89(2), P2Fe1-P1 164.79(5), H-Fe1-Cl1 170(1), C29-Fe1-Cl1 100.1(2), N1-Fe1-Cl1 87.7(1).

The chloro(hydrido) complexes (S,S)-9 and (S)-10 were tested as catalysts for the APH of selected ketones in THF in the presence of base (Scheme 4). These systems were found to be very active under the conditions of 10 bar of $\mathrm{H}_{2}, 50^{\circ} \mathrm{C}$, of ketone: catalyst: $\mathrm{KOtBu}=$ 1000:1:10; oxygen must be rigorously excluded in the preparation of the catalyst solution and its injection into the purged pressure reactor. The complex (S)-10 containing one substituent on the backbone of the ligand when tested for the APH of acetophenone gave low to moderate enantioselectivity depending on the reaction conditions (50$80 \%$ ). Fortunately $(S, S)-9$ with two phenyl groups on the backbone yields excellent results, quantitatively reducing a variety of ketones to the (S)-alcohols in high e.e. (Table 1, Figure 4). The second phenyl substituent on the ligand backbone of this complex is clearly important to lock the $\mathrm{PPh}_{2}$ group into a rigid, asymmetric structure; the complex C (Figure 1) also has this feature, and both (S,S)-9 and $\mathbf{C}$ have similar activity and are much more enantioselective than $(S)-10$ and other complexes that just have one substituent next to the $\mathrm{NH} .{ }^{[13]}$<smiles>[R]C(=O)c1ccc([R])cc1</smiles>

Scheme 4. The APH of ketones

Table 1. Results of ketone APH as in Scheme 4 with catalyst $(S, S)-9 .{ }^{[a]}$ 
<smiles>CC(=O)c1ccc2ccccc2c1</smiles>

12 $99 \%$ $90 \mathrm{~min}$ $90 \%(S)$<smiles>CC(=O)c1cccc2ccccc12</smiles><smiles>CC(=O)c1ccco1</smiles>
13 $61 \%$ 90 min $90 \%(S)$ $99 \%$ $60 \mathrm{~min}$<smiles>CC(=O)C(C)C</smiles><smiles>CC(=O)C(C)(C)C</smiles>
15 $99 \%$ 60 min $45 \%(R)$

Figure 4. Other ketones reduced by the (S,S)-9 system (except 16).

The difference in position of the acetyl functionality in acetylnaphthone substrates 12 and 13 affected the number of turnovers in 90 min., 1000 TON for 12 vs 610 for 13, while both were reduced in high e.e. $(R)-1-(1$-naphthyl)ethanol has been used to desymmetrize prochiral 3-substituted glutaric anhydrides, giving the chiral building blocks for (+)-compactin, an HMG-CoA-reductase inhibitor. ${ }^{[23]}$ 2-Acetylfuran (14) was reduced quantitatively to the alcohol with $95 \%$ e.e. $(S)$. This chiral product can be used for the synthesis of macrosphelide $B$, shown to be a strong inhibitor for the adhesion of human leukemia HL-60 cells to human umbilical-vein cells. ${ }^{[24]} 3$-Methylbutan-2-one (15), a challenging aliphatic ketone, was successfully reduced quantitatively but with low enantioselectivity, and surprisingly, with the $(R)$-alcohol in excess. Oddly the very similar system with catalyst C provided the (S)-alcohol in $46 \%$ e.e. ${ }^{[11]}$ The even more challenging 3,3-dimethylbutan-2-one (16) proved to be too sterically crowded for $(S, S)-9$ to reduce. Further exploration of the activity and enantioselectivity of $(\boldsymbol{S}, \boldsymbol{S})-\mathbf{9}$ is currently in progress.

Previous work has provided strong evidence for a mer $\mathrm{FeH}_{2}(\mathrm{CO}) \mathrm{L}$ structure with trans dihydrides, where $\mathrm{L}$ is a P-NH-P ligand, as the active catalyst that transfers a hydride to the ketone. ${ }^{[10 \mathrm{~b}, 10 \mathrm{e}, 10 \mathrm{~g} \text {, }}$ ${ }^{12 c]}$ The transition state structure producing (S)-1-phenylethanol by attack of a hydride in trans-FeH $\mathrm{H}_{2}(\mathrm{CO})((\boldsymbol{S}, \boldsymbol{S})-\mathbf{1})$ on acetophenone was calculated using DFT (Figure 5) and found to be $1.6 \mathrm{kcal} / \mathrm{mol}$ lower in energy than that producing the $(R)$ alcohol (Supporting information). In this structure the phenyl group of the ketone lies over the isopropyl groups while the smaller methyl group is against a phenyl of the $\mathrm{PPh}_{2}$, that is locked into position by the adjacent asymmetric array of phenyl groups which also lock the five-membered -PCHPhCHPhNHFe- ring by taking the favourable equatorial positions.

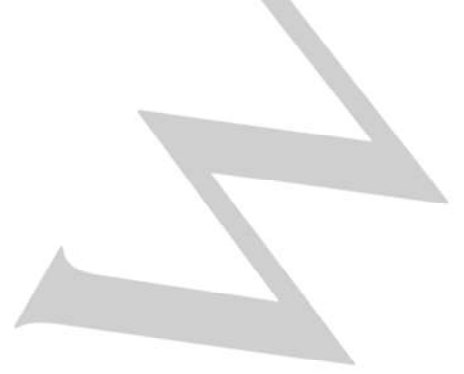

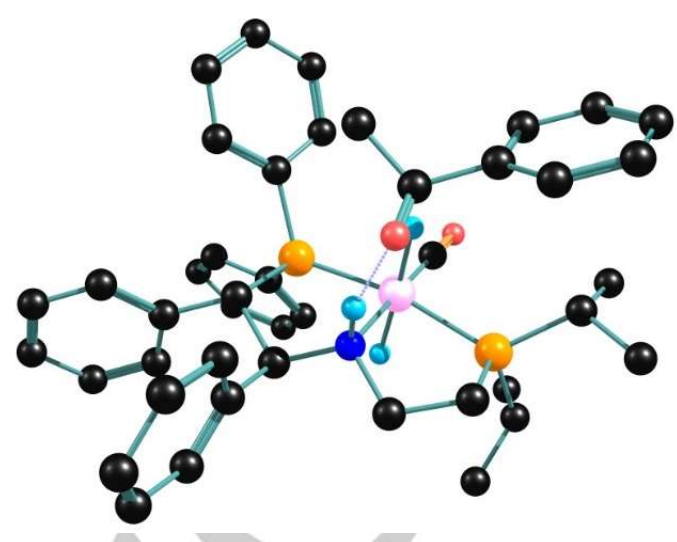

Figure 5. The transition state structure of the transfer of a hydride from the dihydride of the $(S, S)-9$ system to acetophenone to give the $(S)$ alcohol. Hydrogens on the ketone and on the ligand other than the $\mathrm{NH}$ have been deleted for clarity.

In conclusion, a new method of ligand synthesis has been successfully utilized to produce four new iron dichloride complexes and four new iron hydride complexes, one of which shows great promise for the enantioselective reduction of ketones. The use of the well defined precatalyst $(\mathbf{S}, \mathbf{S})-9$ allows the quantitative APH of a variety of ketones to enantiomerically enriched alcohols in up to $96 \%$ e.e. Ortho-chloroacetophenone, 1-acetylnaphthone, and 2acetylfuran have been reduced quantitatively with high enantioselectivities to alcohols of interest to the pharmaceutical industry. We have disclosed a versatile method that allows the synthesis of a wide range of catalyst structures, a flexibility often needed when matching the catalyst with the substrate to attain high levels of enantioselection.

\section{Experimental Section}

See the Supporting Information.

\section{Acknowledgements}

R.H.M. thanks NSERC Canada for a Discovery grant and the Canada Council for the Arts for a Killam Fellowship. This work was also made possible by the SCICOMP NMR facilities provided by the Canada Foundation for Innovation, project number 19119, and the Ontario Ministry of Research, Innovation and Science. The Deutscher Akademischer Austauschdienst (DAAD) is thanked for support for A.P. Calculations were performed using the facilities of SHARCNET and Scinet of Compute/Calcul Canada.

Keywords: Homogeneous hydrogenation • asymmetric synthesis $\bullet$ iron catalysts $\bullet$ ketone reduction $\cdot$ pincer ligand synthesis 
[1] R. Noyori, T. Ohkuma, Angew. Chem. Int. Ed. 2001, 40, 40-73.

[2] J.-H. Xie, D.-H. Bao, Q.-L. Zhou, Synthesis 2015, 47, 460-471.

[3] a) T. Ollevier, H. Keipour, in Iron Catalysis, Vol. 50 (Ed.: E. Bauer), Springer-Verlag Berlin, Berlin, 2015, pp. 259-309; b) Y.-Y. Li, S.-L. Yu, W.-Y. Shen, J.-X. Gao, Acc. Chem. Res. 2015, 48, 25872598; c) R. H. Morris, Acc. Chem. Res. 2015, 48, 1494-1502. [4] Y.-Y. Li, S.-L. Yu, X.-F. Wu, J.-L. Xiao, W.-Y. Shen, Z.-R. Dong, J.-X. Gao, J. Am. Chem. Soc. 2014, 136, 4031-4039.

[5] a) C. Sui-Seng, F. Freutel, A. J. Lough, R. H. Morris, Angew. Chem. Int. Ed. 2008, 47, 940-943; b) C. Sui-Seng, F. N. Haque, A. Hadzovic, A.-M. Pütz, V. Reuss, N. Meyer, A. J. Lough, M. Z.-D. Iuliis, R. H. Morris, Inorg. Chem. 2009, 48, 735-743; c) W. Zuo, S. Tauer, D. E. Prokopchuk, R. H. Morris, Organometallics 2014, 33, $5791-5801$

[6] W. Zuo, A. J. Lough, Y. Li, R. H. Morris, Science 2013, 342, 1080-1083.

[7] a) A. Berkessel, S. Reichau, A. von der Hoh, N. Leconte, J. M. Neudorfl, Organometallics 2011, 30, 3880-3887; b) D. S. Merel, S. Gaillard, T. R. Ward, J. L. Renaud, Catal. Lett. 2016, 146, 564-569; c) P. Gajewski, M. Renom-Carrasco, S. V. Facchini, L. Pignataro, L. Lefort, J. G. de Vries, R. Ferraccioli, A. Forni, U. Piarulli, C. Gennari, Eur. J. Org. Chem. 2015, 1887-1893; d) P. Gajewski, M. RenomCarrasco, S. V. Facchini, L. Pignataro, L. Lefort, J. G. de Vries, R. Ferraccioli, U. Piarulli, C. Gennari, Eur. J. Org. Chem. 2015, 55265536.

[8] S. Fleischer, S. L. Zhou, S. Werkmeister, K. Junge, M. Beller, Chem. - Eur. J. 2013, 19, 4997-5003.

[9] R. Langer, G. Leitus, Y. Ben-David, D. Milstein, Angew. Chem. Int. Ed. 2011, 50, 2120-2124.

[10] a) E. Alberico, P. Sponholz, C. Cordes, M. Nielsen, H. J. Drexler, W. Baumann, H. Junge, M. Beller, Angew. Chem. Int. Ed. 2013, 52, 14162-14166; b) S. Chakraborty, H. Dai, P. Bhattacharya, N. T. Fairweather, M. S. Gibson, J. A. Krause, H. Guan, J. Am. Chem. Soc. 2014, 136, 7869-7872; c) S. Chakraborty, W. W. Brennessel, W. D. Jones, J. Am. Chem. Soc. 2014, 136, 8564-8567; d) S. Werkmeister, K. Junge, B. Wendt, E. Alberico, H. Jiao, W. Baumann, H. Junge, F. Gallou, M. Beller, Angew. Chem. Int. Ed. 2014, 53, 8722-8726; e) E. A. Bielinski, P. O. Lagaditis, Y. Y. Zhang, B. Q. Mercado, C. Wurtele, W. H. Bernskoetter, N. Hazari, S. Schneider, J. Am. Chem. Soc. 2014, 136, 10234-10237; f) S. Chakraborty, P. O. Lagaditis, M. Forster, E. A. Bielinski, N. Hazari, M. C. Holthausen, W. D. Jones, S. Schneider, ACS Catal. 2014, 4, 3994-4003; g) C. Bornschein, S. Werkmeister, B. Wendt, H. Jiao, E. Alberico, W. Baumann, H. Junge, K. Junge, M. Beller, Nat.
Commun. 2014, 5, Article No. 4111; h) M. Pena-Lopez, H. Neumann, M. Beller, ChemCatChem 2015, 7, 865-871; i) F. Schneck, M. Assmann, M. Balmer, K. Harms, R. Langer, Organometallics 2016, 35, 1931-1943; j) R. Xu, S. Chakraborty, S. M. Bellows, H. Yuan, T. R. Cundari, W. D. Jones, ACS Catal. 2016, 6, 2127-2135; k) U. Jayarathne, Y. Zhang, N. Hazari, W. H. Bernskoetter, Organometallics 2017, 36, 409-416.

[11] P. O. Lagaditis, P. E. Sues, J. F. Sonnenberg, K. Y. Wan, A. J. Lough, R. H. Morris, J. Am. Chem. Soc. 2014, 136, 1367-1380. [12] a) R. Noyori, M. Yamakawa, S. Hashiguchi, J. Org. Chem. 2001, 66, 7931-7944; b) B. Zhao, Z. Han, K. Ding, Angew. Chem. Int. Ed. 2013, 52, 4744-4788; c) J. F. Sonnenberg, P. E. Sues, K. Y. Wan, R. H. Morris, ACS Catal. 2017, 7, 316-326.

[13] J. F. Sonnenberg, P. O. Lagaditis, A. J. Lough, R. H. Morris, Organometallics 2014, 33, 6452-6465; correction: Organometallics 2016, 6435, 2772-2772.

[14] A. Zirakzadeh, K. Kirchner, A. Roller, B. Stöger, M. Widhalm, R. H. Morris, Organometallics 2016, 35, 3781-3787.

[15] C. Schroder-Holzhacker, N. Gorgas, B. Stoger, K. Kirchner, Monat. Fur Chem. 2016, 147, 1023-1030.

[16] P. O. Lagaditis, A. A. Mikhailine, A. J. Lough, R. H. Morris, Inorg. Chem. 2010, 49, 1094-1102.

[17] A. F. Abdel-Magid, K. G. Carson, B. D. Harris, C. A. Maryanoff, R. D. Shah, J. Org. Chem. 1996, 61, 3849-3862.

[18] a) H. Y. Su, Y. Song, M. S. Taylor, Org. Biomolec. Chem. 2016, 14, 5665-5672; b) P. Chen, X. Su, W. Zhou, Y. J. Xiao, J. L. Zhang, Tetrahedron 2016, 72, 2700-2706; c) R. W. Guo, S. M. Lu, X. H.

Chen, C. W. Tsang, W. L. Jia, C. Sui-Seng, D. Amoroso, K. AbdurRashid, J. Org. Chem. 2010, 75, 937-940.

[19] P. E. Sues, A. J. Lough, R. H. Morris, Organometallics 2011, 30, 4418-4431.

[20] I. Koehne, T. J. Schmeier, E. A. Bielinski, C. J. Pan, P. O. Lagaditis, W. H. Bernskoetter, M. K. Takase, C. Würtele, N. Hazari, S. Schneider, Inorg. Chem. 2014, 53, 2133-2143.

[21] V. Parekh, J. A. Ramsden, M. Wills, Catal. Sci. Technol. 2012, $2,406-414$.

[22] T. Eixelsberger, J. M. Woodley, B. Nidetzky, R. Kratzer, Biotechnol. Bioeng. 2013, 100, 2311-2231.

[23] a) P. D. Theisen, C. H. Heathcock, J. Org. Chem. 1988, 53, 2374-2378; b) O. Dirat, C. Kouklovsky, Y. Langlois, J. Org. Chem. 1998, 63, 6634-6642.

[24] Y. Kobayashi, B. G. Kumar, T. Kurachi, Tetrahedron Lett. 2000, $41,1559-1563$. 
Entry for the Table of Contents (Please choose one layout)

\section{COMMUNICATION}

Highly enantioselective and active iron catalyst. The reductive amination of $\alpha$-dialkylphosphine acetaldehydes with enantiopure $\beta$ aminophosphines is a new, versatile route to unsymmetrical tridentate (pincer) ligands P-NH-P'. Several prochiral aryl ketones are reduced to the (S)-alcohols, usually in e.e. greater than $90 \%$, using $0.1 \mathrm{~mol} \%$ of an iron complex mer- $\mathrm{FeCl}(\mathrm{H})(\mathrm{CO})(\mathrm{P}$ $\mathrm{NH}-\mathrm{P}^{\prime}$ ) at 5 to 10 bar $\mathrm{H}_{2}$ and $50^{\circ} \mathrm{C}$.
Samantha A. M. Smith, Paraskevi O. Lagaditis, Anne Lüpke, Alan J, Lough, Robert H. Morris*

Page No. - Page No.

Unsymmetrical Iron P-NH-P' Catalysts for the Asymmetric Pressure Hydrogenation of Aryl Ketones 


\section{Contents}

General procedures.

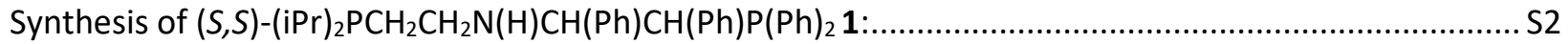

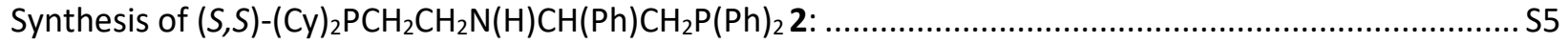

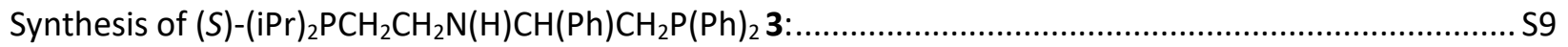

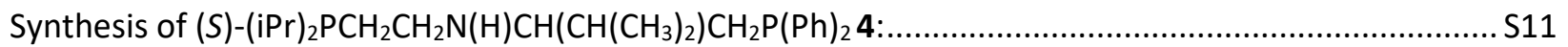

Synthesis of trans- $\mathrm{Fe}(\mathrm{Cl})_{2}(\mathrm{CO})\left[(\mathrm{S}, \mathrm{S})-(\mathrm{iPr})_{2} \mathrm{PCH}_{2} \mathrm{CH}_{2} \mathrm{~N}(\mathrm{H}) \mathrm{CH}(\mathrm{Ph}) \mathrm{CH}(\mathrm{Ph}) \mathrm{P}(\mathrm{Ph})_{2}\right](\mathrm{S}, \mathrm{S})-5: \ldots \ldots \ldots \ldots \ldots \ldots \ldots \ldots . . . . . . . . . . . . . .515$

Synthesis of trans-Fe(Cl) $)_{2}(\mathrm{CO})\left[(\mathrm{S}, \mathrm{S})-(\mathrm{Cy})_{2} \mathrm{PCH}_{2} \mathrm{CH}_{2} \mathrm{~N}(\mathrm{H}) \mathrm{CH}(\mathrm{Ph}) \mathrm{CH}(\mathrm{Ph}) \mathrm{P}(\mathrm{Ph})_{2}\right](\mathrm{S}, \mathrm{S})-6$............................... 119

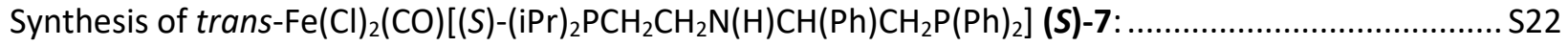

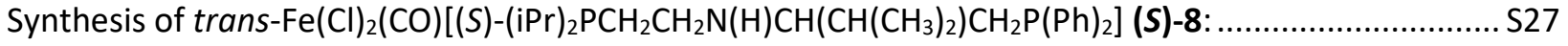

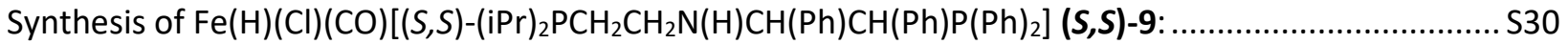

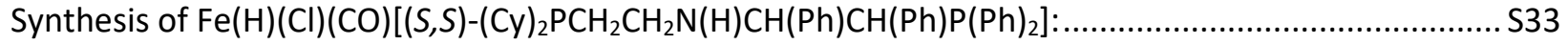

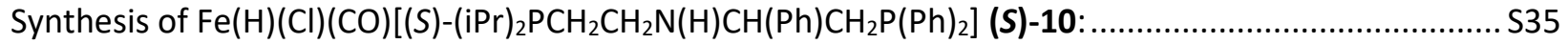

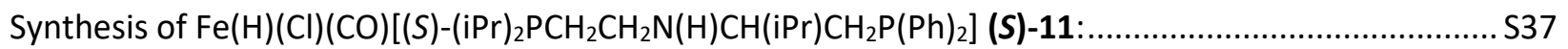

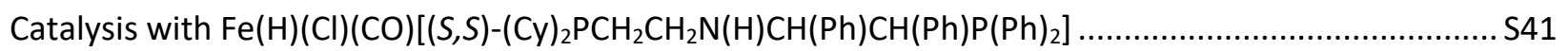

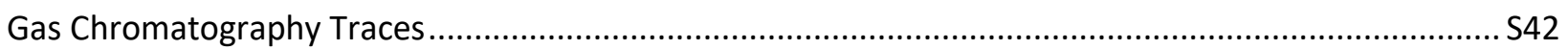

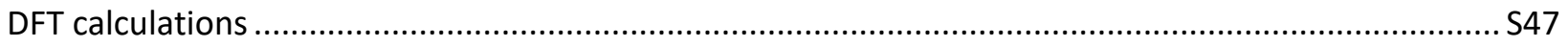

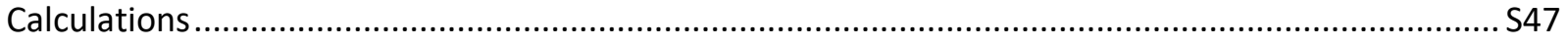

Reaction coordinate diagram for hydride transfer to the ketone................................................... S48

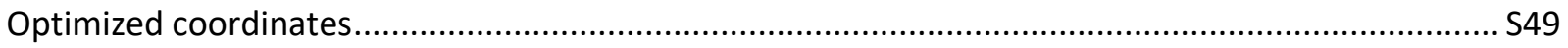

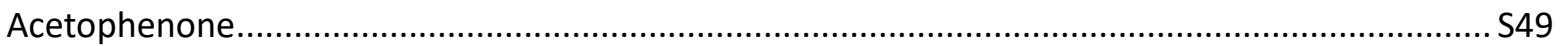

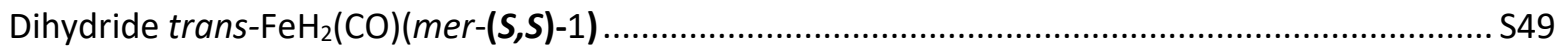

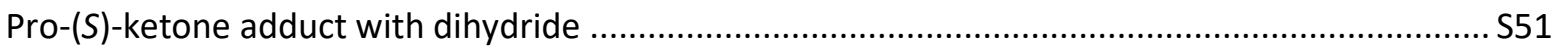

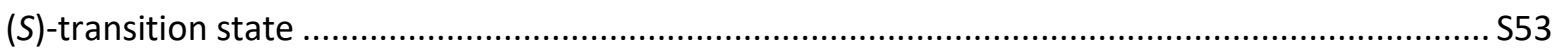

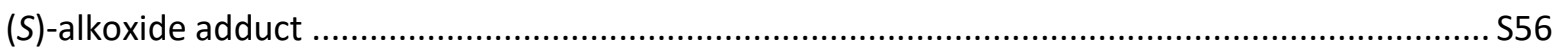

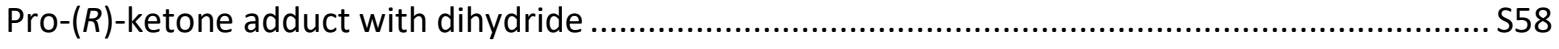

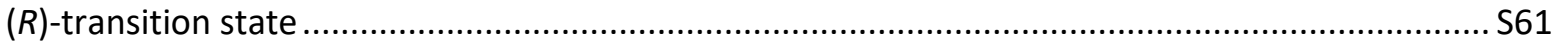

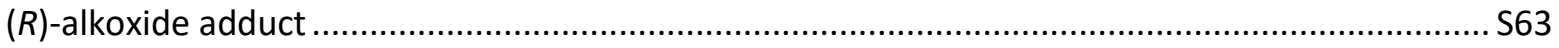




\section{General procedures.}

All procedures and manipulations were performed under an argon or nitrogen atmosphere using standard Schlenk-line and glove box techniques unless stated otherwise. All solvents were degassed and dried using standard procedures prior to all manipulations and reactions unless stated otherwise. Deuterated solvents were purchased from Cambridge Isotope Laboratories or Sigma Aldrich, degassed, and dried over activated molecular sieves prior to use. (1S,2S)-2-(diphenylphosphino)-1,2-diphenylethylamine was purchased from ACROS Organics, (S)-1-(diphenylphosphino)-2-phenylethylamine, and (S)-1(diphenylphosphino)-3-methyl-2-butylamine was purchased from Alfa Aesar. All other reagents were purchased from commercial sources and utilized without further purification. NMR spectra were recorded at ambient temperature and pressure using a Agilent $500 \mathrm{MHz}$ spectrometer with a OneNMR $\mathrm{H} / \mathrm{F}\{\mathrm{X}\}$ Probe (500 MHz for ${ }^{1} \mathrm{H}, 126 \mathrm{MHz}$ for ${ }^{13} \mathrm{C}$ and $202 \mathrm{MHz}$ for ${ }^{31} \mathrm{P}$ ), or an Agilent DD2-600 MHz spectrometer (600 $\mathrm{MHz}$ for ${ }^{1} \mathrm{H}, 151 \mathrm{MHz}$ for ${ }^{13} \mathrm{C}$ and $243 \mathrm{MHz}$ for ${ }^{31} \mathrm{P}$ ) unless stated otherwise. The ${ }^{1} \mathrm{H}$ and ${ }^{13} \mathrm{C}$ NMR were measured relative to partially deuterated solvent peaks but are reported relative to tetramethylsilane (TMS). All ${ }^{31} \mathrm{P}$ chemical shifts were measured relative to $85 \%$ phosphoric acid as an external reference. Gas Chromatography was done on a Shimadzu GC-2014 Gas Chromatograph equipped with a chiral column (CP chirasil-Dex CB $25 \mathrm{~m} \times 2.5 \mathrm{~mm} \times 2.5 \mu \mathrm{m}$ ) to determine substrate conversion and enantiopurity. Hydrogen and nitrogen gases were used as the mobile phase. All of the hydrogenation reactions were performed in a $50 \mathrm{~mL}$ stainless steel Parr Hydrogenation reactor at constant temperatures and pressures. The temperature was maintained at $50{ }^{\circ} \mathrm{C}$ using a constant temperature water bath and was purged of oxygen by flushing the reactor several times with $10 \mathrm{~atm}$ of $\mathrm{H}_{2(\mathrm{~g})}$. The elemental analyses were performed on a Perkin-Elmer $2400 \mathrm{CHN}$ elemental analyzer.

\section{Synthesis of $(\mathrm{S}, \mathrm{S})-(\mathrm{iPr})_{2} \mathrm{PCH}_{2} \mathrm{CH}_{2} \mathrm{~N}(\mathrm{H}) \mathrm{CH}(\mathrm{Ph}) \mathrm{CH}(\mathrm{Ph}) \mathrm{P}(\mathrm{Ph})_{2} 1$ :}

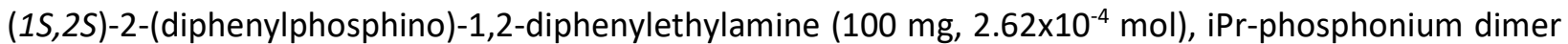

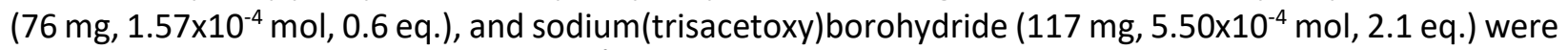
mixed in THF (10 mL) and stirred over $3 \AA \mathrm{MS}$ for 16 hours. Solvent was removed in vacuo and the residue was redissolved in dichloromethane. The solution was transferred into a Schlenk flask and placed on a Schlenk line under argon. A solution of saturated $\mathrm{NH}_{4} \mathrm{Cl}_{(\text {aq) }}$ and a second solution of distilled water were degassed and placed under argon. An air-free wash with the $\mathrm{NH}_{4} \mathrm{Cl}$ solution $(2 \times 10 \mathrm{~mL})$ then with water $(2 \times 10 \mathrm{~mL})$. The solution was dried over $\mathrm{MgSO}_{4}$, then filtered. The solvent was removed to give a colourless solid. Yield $80 \mathrm{mg}(58 \%) .{ }^{1} \mathrm{H}$ NMR $\left(600 \mathrm{MHz}, \mathrm{CD}_{2} \mathrm{Cl}_{2}\right) \delta$ : 0.87-0.96 $\left(\mathrm{m}, 12 \mathrm{H}, \mathrm{C}\left(\mathrm{CH}_{3}\right)_{2}\right), 1.57 \mathrm{ppm}(\mathrm{m}, 2 \mathrm{H}$, $\left.\mathrm{CH}\left(\mathrm{CH}_{3}\right)_{2}\right), 2.34 \mathrm{ppm}(\mathrm{d}, 1 \mathrm{H}, \mathrm{CH}), 2.46 \mathrm{ppm}(\mathrm{s}, 1 \mathrm{H}, \mathrm{CH}), 4.05 \mathrm{ppm}\left(\mathrm{m}, 2 \mathrm{H}, \mathrm{CH}_{2}\right), 6.87-7.80(\mathrm{~m}, 20 \mathrm{H}) .{ }^{31} \mathrm{P}$ $\left\{{ }^{1} \mathrm{H}\right\} \operatorname{NMR}\left(242 \mathrm{MHz}, \mathrm{CD}_{2} \mathrm{Cl}_{2}\right) \delta$ : $-2.04 \mathrm{ppm}\left(\mathrm{s}, \mathrm{iPr}_{2} \mathrm{P}\right),-8.50\left(\mathrm{~s}, \mathrm{Ph}_{2} \mathrm{P}\right.$ ). HRMS (ESI-TOF, $\mathrm{CH}_{2} \mathrm{Cl}_{2}$ ) m/z calculated for $\left[\mathrm{C}_{34} \mathrm{H}_{42} \mathrm{NP}_{2}\right]^{+}: 526.2787$, found: 526.2788 . 


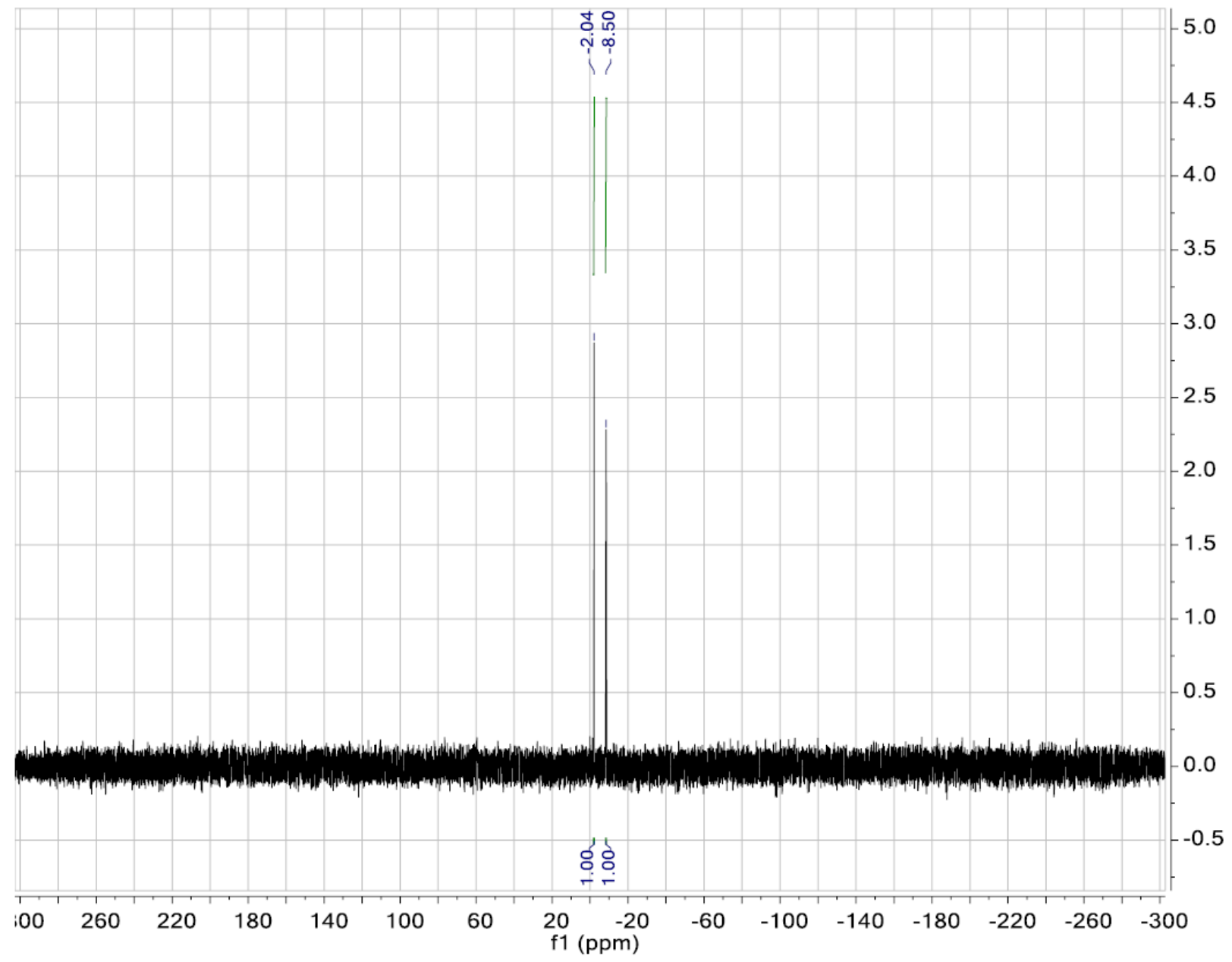

Figure S1: ${ }^{31} \mathrm{P}\left\{{ }^{1} \mathrm{H}\right\}$ NMR spectrum $\left(242 \mathrm{MHz}, \mathrm{CD}_{2} \mathrm{Cl}_{2}\right)$ of $(\mathrm{S}, \mathrm{S})-(\mathrm{PPr})_{2} \mathrm{PCH}_{2} \mathrm{CH}_{2} \mathrm{~N}(\mathrm{H}) \mathrm{CH}(\mathrm{Ph}) \mathrm{CH}(\mathrm{Ph}) \mathrm{P}(\mathrm{Ph})_{2} 1$ 


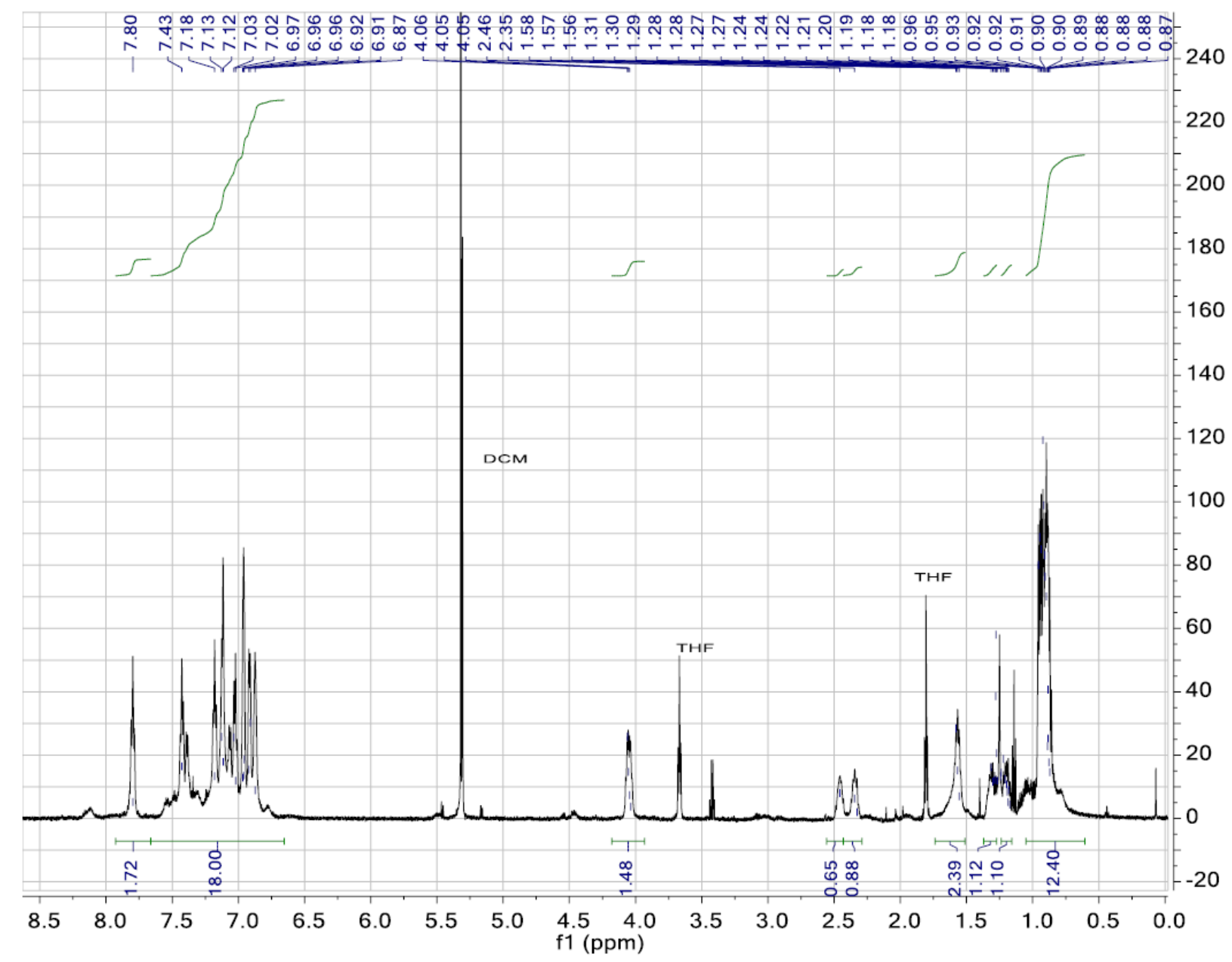

Figure S2: ${ }^{1} \mathrm{H}$ NMR spectrum $\left(600 \mathrm{MHz}, \mathrm{CD}_{2} \mathrm{Cl}_{2}\right)$ of $(\mathrm{S}, \mathrm{S})-(\mathrm{PPr})_{2} \mathrm{PCH}_{2} \mathrm{CH}_{2} \mathrm{~N}(\mathrm{H}) \mathrm{CH}(\mathrm{Ph}) \mathrm{CH}(\mathrm{Ph}) \mathrm{P}(\mathrm{Ph})_{2} 1$ 


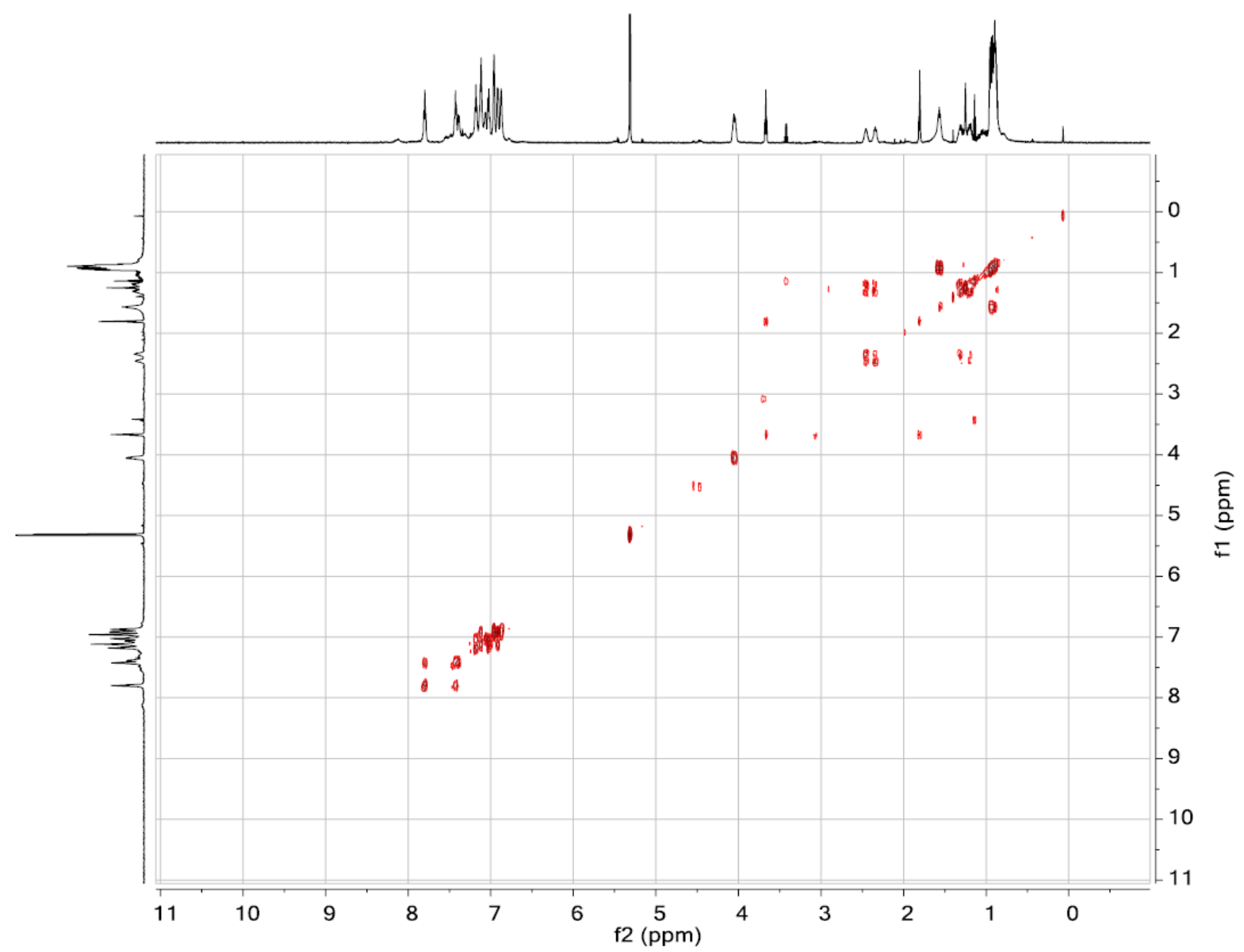

Figure S3: gCOSY $\left(600 \mathrm{MHz}, \mathrm{CD}_{2} \mathrm{Cl}_{2}\right)$ of $(\mathrm{S}, \mathrm{S})-(\mathrm{PPr})_{2} \mathrm{PCH}_{2} \mathrm{CH}_{2} \mathrm{~N}(\mathrm{H}) \mathrm{CH}(\mathrm{Ph}) \mathrm{CH}(\mathrm{Ph}) \mathrm{P}(\mathrm{Ph})_{2} 1$

\section{Synthesis of $(\mathrm{S}, \mathrm{S})-(\mathrm{Cy})_{2} \mathrm{PCH}_{2} \mathrm{CH}_{2} \mathrm{~N}(\mathrm{H}) \mathrm{CH}(\mathrm{Ph}) \mathrm{CH}_{2} \mathrm{P}(\mathrm{Ph})_{2} 2$ :}

Follows the procedure above with (1S,2S)-2-(diphenylphosphino)-1,2-diphenylethylamine (250 $\mathrm{mg}$, $\left.6.55 \times 10^{-4} \mathrm{~mol}\right)$, and $\mathrm{Cy}$-phosphonium dimer $\left(211 \mathrm{mg}, 3.28 \times 10^{-4} \mathrm{~mol}\right)$. This reaction yielded a colourless powder. Yield $108 \mathrm{mg}, 54 \% .{ }^{1} \mathrm{H}$ NMR $\left(500 \mathrm{MHz}, \mathrm{CD}_{2} \mathrm{Cl}_{2}\right)$ 8: 1.01-1.73 (m, $\left.24 \mathrm{H}, \mathrm{C}_{6} \mathrm{H}_{11}+\mathrm{CH}_{2} \mathrm{PCy}\right)$ ), $2.36(\mathrm{bm}$, $1 \mathrm{H}, \mathrm{CHNH}), 2.45(\mathrm{~m}, 1 \mathrm{H}, \mathrm{CHNH}), 4.08(\mathrm{bm}, 2 \mathrm{H}$, overlapping $\mathrm{CH}(\mathrm{Ph}) \times 2), 6.90-7.21(\mathrm{~m}, 14 \mathrm{H}, \mathrm{Ar}-\mathrm{H}), 7.46(\mathrm{~m}$, $3 \mathrm{H}, \mathrm{Ar}-H), 7.83(\mathrm{~m}, 3 \mathrm{H}, \mathrm{Ar}-\mathrm{H}) .{ }^{13} \mathrm{C}\left\{{ }^{1} \mathrm{H}\right\} \mathrm{NMR}\left(\mathrm{CD}_{2} \mathrm{Cl}_{2}, 124 \mathrm{MHz}\right): 133.93\left(\mathrm{~d},{ }^{2} J_{\mathrm{CP}}=20 \mathrm{~Hz}, \mathrm{C}_{\mathrm{Ar}}\right), 133,76\left(\mathrm{~d}, J_{\mathrm{CP}}=\right.$ $\left.20 \mathrm{~Hz}, C_{\mathrm{Ar}}\right), 130.5\left(C_{\mathrm{Ar}}\right), 130.4\left(C_{\mathrm{Ar}}\right), 128.9\left(C_{\mathrm{Ar}}\right), 128.6\left(C_{\mathrm{Ar}}\right), 128.5\left(C_{\mathrm{Ar}}\right), 128.3\left(C_{\mathrm{Ar}}\right), 128.0\left(C_{\mathrm{Ar}}\right), 127.7\left(C_{\mathrm{Ar}}\right)$, 127.4 $\left(C_{\mathrm{Ar}}\right), 127.3\left(\mathrm{PC} C_{\mathrm{Ar}}\right), 126.7\left(C_{\mathrm{Ar}}\right), 125.9\left(C_{\mathrm{Ar}}\right), 65.6\left(\mathrm{~d}, J_{\mathrm{CP}}=20 \mathrm{~Hz}, \mathrm{CHPh}\right), 51.9\left(\mathrm{~d}, J_{\mathrm{CP}}=16 \mathrm{~Hz}, \mathrm{CHPh}\right), 46.3$ (d overlapped, $\mathrm{CH}_{2} \mathrm{CH}_{2} \mathrm{PiPr}_{2}$ ), 46.3 (d overlapped, $\mathrm{CH}_{2} \mathrm{CH}_{2} \mathrm{PiPr}_{2}$ ), $33.2\left(\mathrm{~m}, \mathrm{C}_{\mathrm{Cy}}\right), 30.2\left(\mathrm{~m}, \mathrm{C}_{\mathrm{Cy}}\right), 28.9\left(\mathrm{C}_{\mathrm{Cy}}\right), 27.3$ $\left(\mathrm{d}, J_{\mathrm{CP}}=12 \mathrm{~Hz}, C_{\mathrm{Cy}}\right), 27.2\left(\mathrm{CH}_{2} \mathrm{PC} \mathrm{y}_{2}\right), 26.5\left(C_{\mathrm{cy}}\right) .{ }^{31} \mathrm{P}\left\{{ }^{1} \mathrm{H}\right\} \mathrm{NMR}\left(202 \mathrm{MHz}, \mathrm{CD}_{2} \mathrm{Cl}_{2}\right)$ 8: -8.55 ppm $\left(\mathrm{bs}, \mathrm{Cy}_{2} P\right)$, $10.73\left(\mathrm{~s}, \mathrm{Ph}_{2} P\right.$ ). HRMS (ESI-TOF, $\mathrm{CH}_{2} \mathrm{Cl}_{2}$ ) m/z calculated for $\left[\mathrm{C}_{40} \mathrm{H}_{50} \mathrm{NP}_{2}\right]^{+}: 607.3447$, found: 607.3430 . 


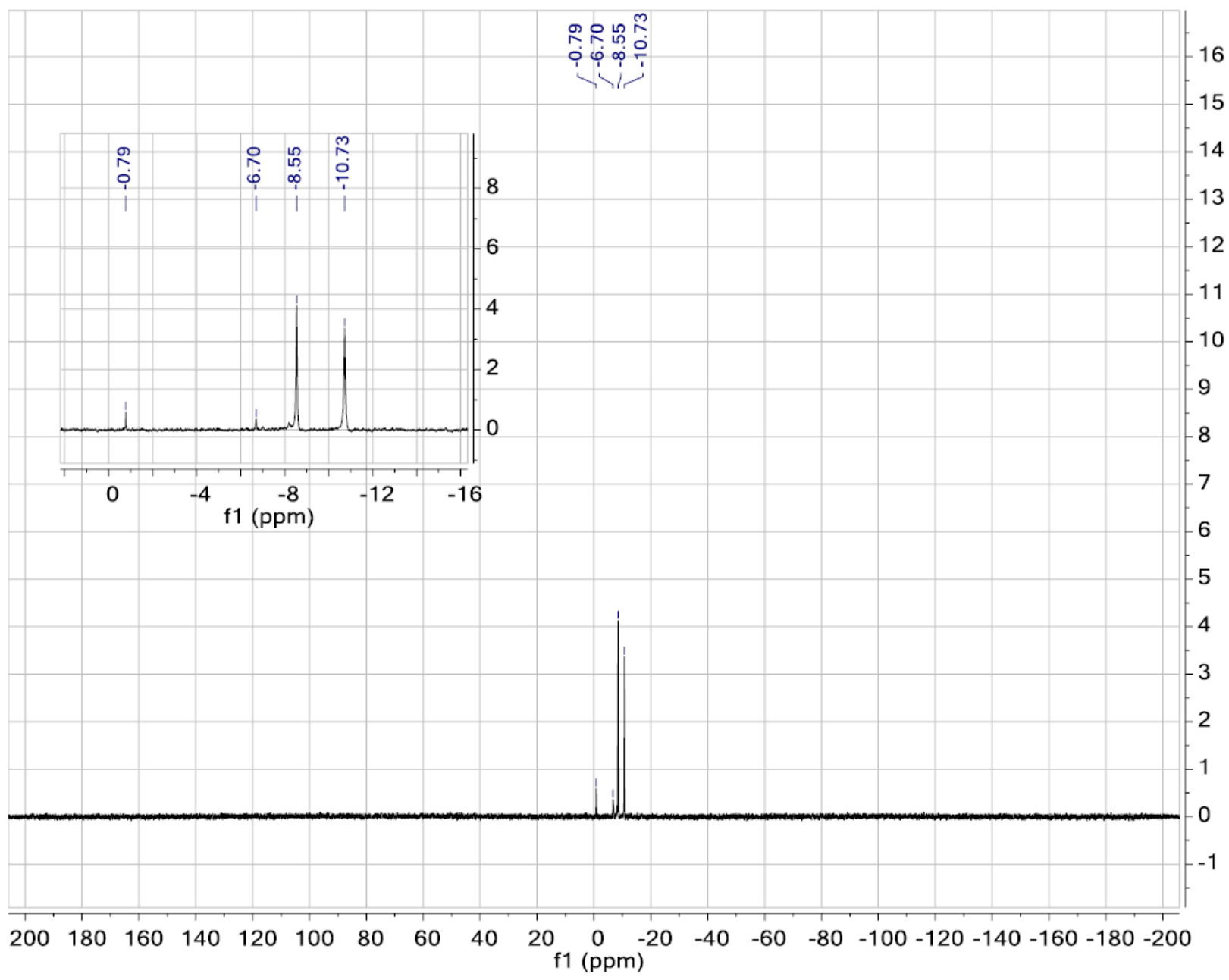

Figure S4: ${ }^{31} \mathrm{P}\left\{{ }^{1} \mathrm{H}\right\}$ NMR spectrum $\left(202 \mathrm{MHz}, \mathrm{CD}_{2} \mathrm{Cl}_{2}\right)$ of $(\mathrm{S}, \mathrm{S})-(\mathrm{Cy}){ }_{2} \mathrm{PCH}_{2} \mathrm{CH}_{2} \mathrm{~N}(\mathrm{H}) \mathrm{CH}(\mathrm{Ph}) \mathrm{CH}(\mathrm{Ph}) \mathrm{P}(\mathrm{Ph})_{2} 2$ 


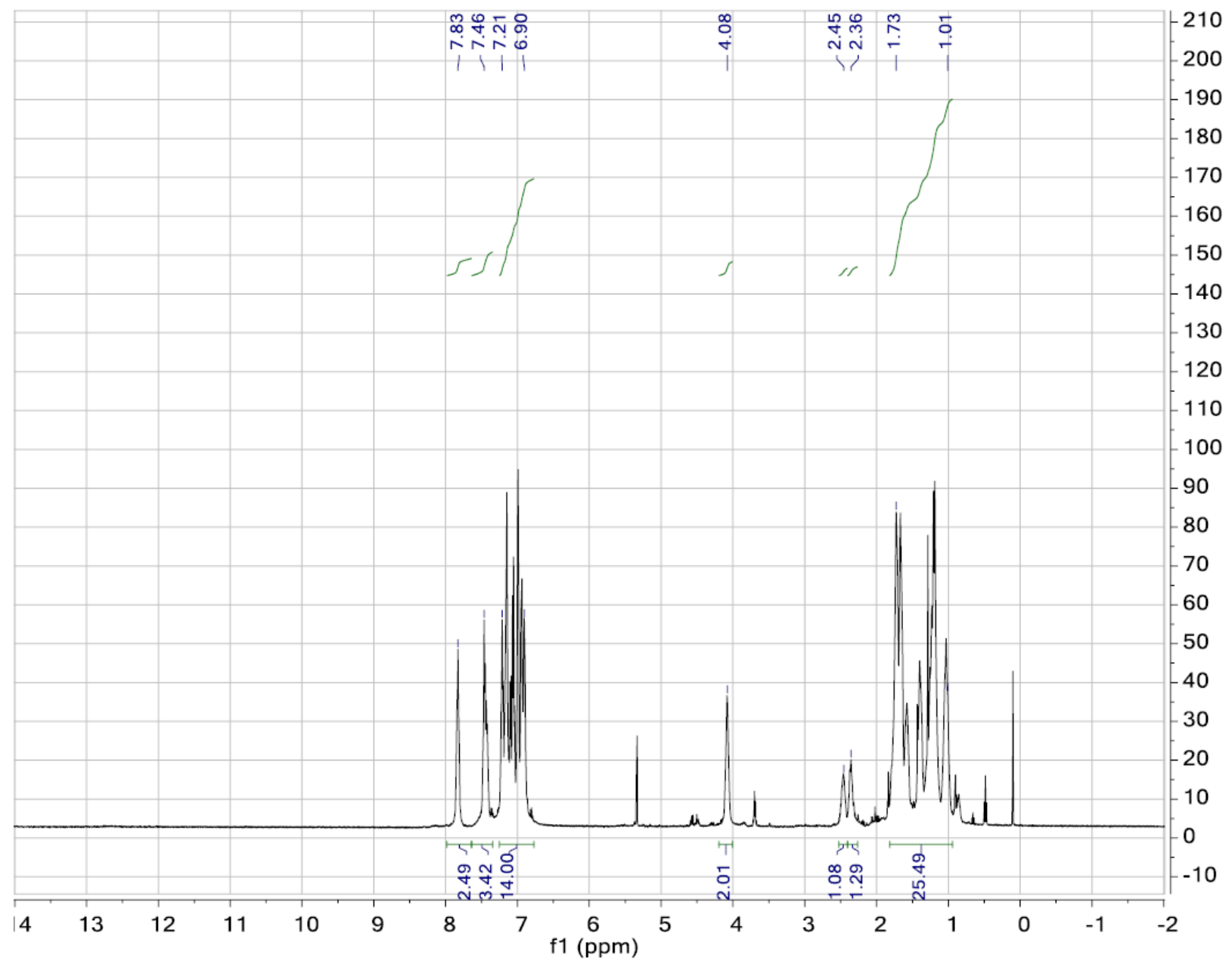

Figure S5: ${ }^{1} \mathrm{H}$ NMR spectrum $\left(500 \mathrm{MHz}, \mathrm{CD}_{2} \mathrm{Cl}_{2}\right)$ of $(\mathrm{S}, \mathrm{S})-(\mathrm{Cy})_{2} \mathrm{PCH}_{2} \mathrm{CH}_{2} \mathrm{~N}(\mathrm{H}) \mathrm{CH}(\mathrm{Ph}) \mathrm{CH}(\mathrm{Ph}) \mathrm{P}(\mathrm{Ph})_{2} 2$ 


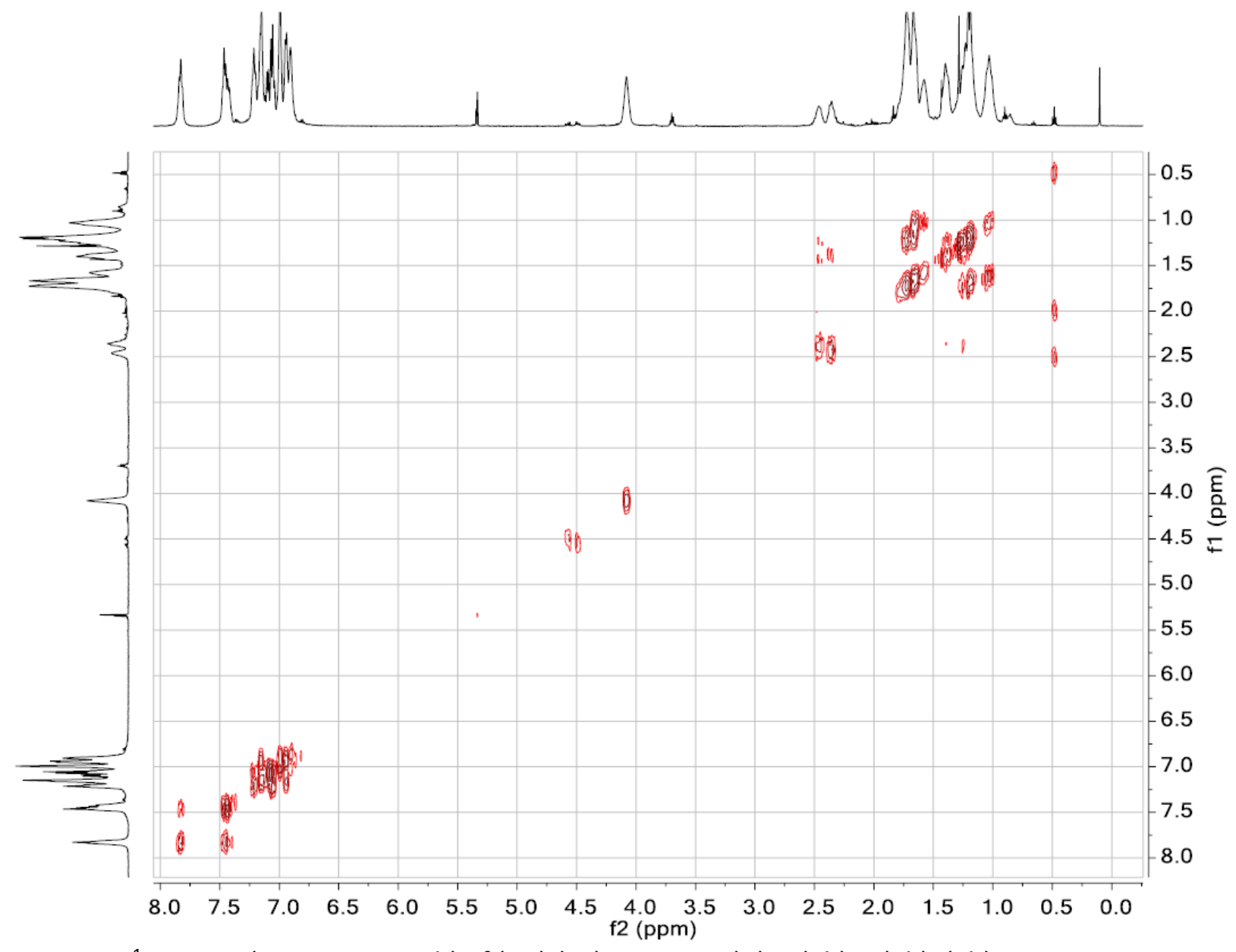

Figure S6: ${ }^{1} \mathrm{H}$ gCOSY $\left(500 \mathrm{MHz}, \mathrm{CD}_{2} \mathrm{Cl}_{2}\right)$ of $(\mathrm{S}, \mathrm{S})-(\mathrm{Cy})_{2} \mathrm{PCH}_{2} \mathrm{CH} \mathrm{H}_{2} \mathrm{~N}(\mathrm{H}) \mathrm{CH}(\mathrm{Ph}) \mathrm{CH}(\mathrm{Ph}) \mathrm{P}(\mathrm{Ph})_{2} 2$ 


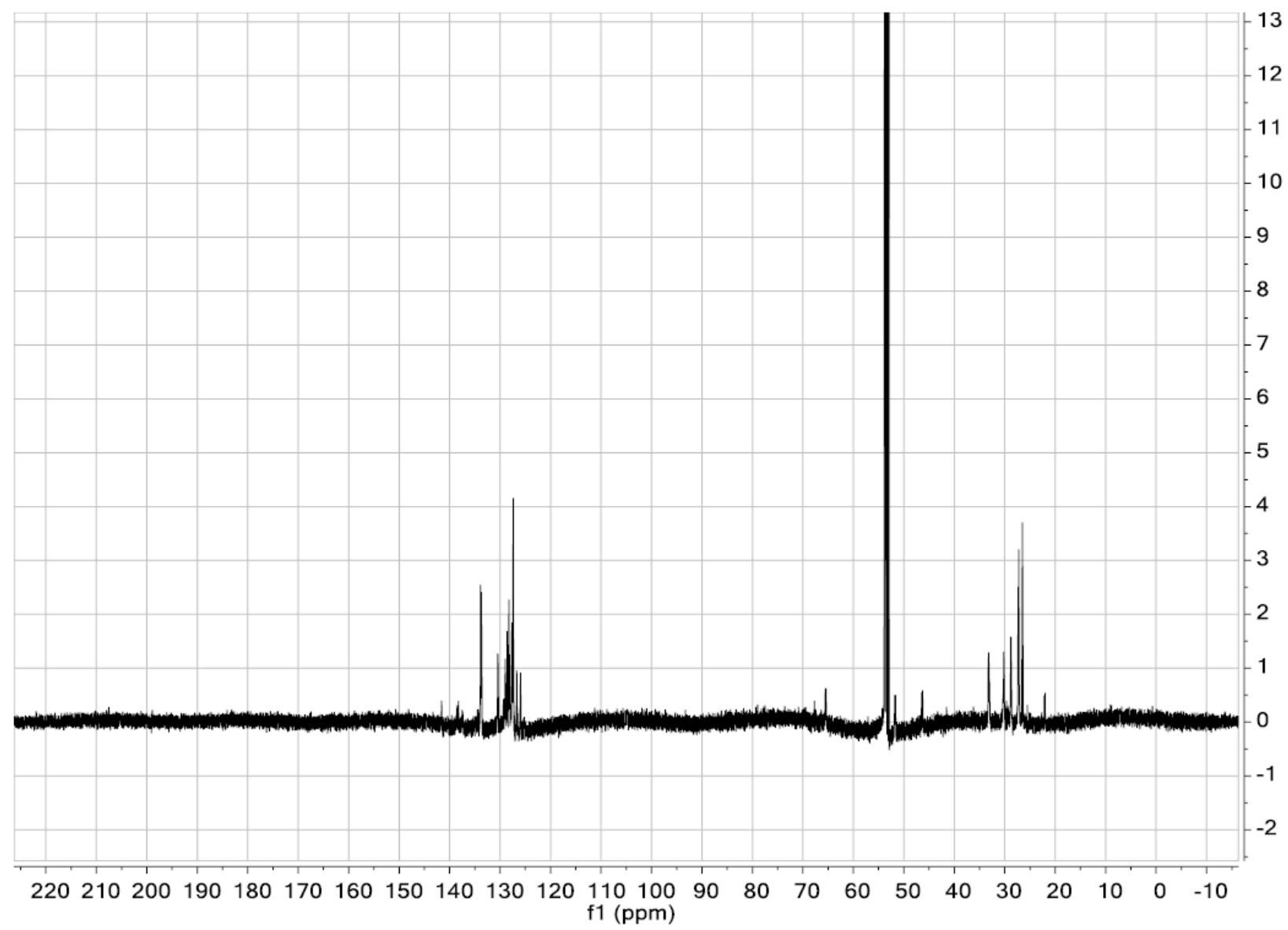

Figure S7: ${ }^{13} \mathrm{C}\left\{{ }^{1} \mathrm{H}\right\}\left(125 \mathrm{MHz}, \mathrm{CD}_{2} \mathrm{Cl}_{2}\right)$ of $(\mathrm{S}, \mathrm{S})-(\mathrm{Cy})_{2} \mathrm{PCH}_{2} \mathrm{CH}{ }_{2} \mathrm{~N}(\mathrm{H}) \mathrm{CH}(\mathrm{Ph}) \mathrm{CH}(\mathrm{Ph}) \mathrm{P}(\mathrm{Ph})_{2} 2$

\section{Synthesis of (S)-(iPr) $)_{2} \mathrm{PCH}_{2} \mathrm{CH}_{2} \mathrm{~N}(\mathrm{H}) \mathrm{CH}(\mathrm{Ph}) \mathrm{CH}_{2} \mathrm{P}(\mathrm{Ph})_{2} 3$ :}

Follows the procedure above with (S)-1-(diphenylphosphino)-2-phenylethylamine (100 mg, 3.27x10-4 mol), and iPr-phosphonium dimer (95 mg, $1.96 \times 10^{-4} \mathrm{~mol}, 0.6$ eq.). This reaction yielded a colourless, viscous oil. Yield $110 \mathrm{mg}(75 \%) .{ }^{1} \mathrm{H} \mathrm{NMR}\left(600 \mathrm{MHz}, \mathrm{CD}_{2} \mathrm{Cl}_{2}\right) \delta: 0.87-0.95\left(\mathrm{~m}, 12 \mathrm{H}, \mathrm{C}\left(\mathrm{CH}_{3}\right)_{2}\right), 1.3(\mathrm{~m}, 1 \mathrm{H}$, $\left.\mathrm{CH}\left(\mathrm{CH}_{3}\right)_{2}\right), 1.56\left(\mathrm{~m}, 3 \mathrm{H}, 2 \mathrm{CH}\right.$ plus $\left.\mathrm{CH}\left(\mathrm{CH}_{3}\right)_{2}\right), 2.66(\mathrm{bm}, 1 \mathrm{H}, \mathrm{CH}), 2.79(\mathrm{bm}, 2 \mathrm{H}, \mathrm{CH}), 3.4(\mathrm{bm}, 1 \mathrm{H}, \mathrm{CH}), 4.03$ (bm, $\left.1 \mathrm{H}, \mathrm{CH}_{2}\right), 7.21-7.51(\mathrm{~m}, 15 \mathrm{H}) .{ }^{31} \mathrm{P}\left\{{ }^{1} \mathrm{H}\right\} \mathrm{NMR}\left(242 \mathrm{MHz}, \mathrm{CD}_{2} \mathrm{Cl}_{2}\right)$ 8: -0.71 ppm (bs, $\left.\operatorname{Pr}_{2} \mathrm{P}\right),-22.76$ (s, $\mathrm{Ph}_{2} P$ ). HRMS (ESI-TOF, $\mathrm{CH}_{2} \mathrm{Cl}_{2}$ ) m/z calculated for $\left[\mathrm{C}_{28} \mathrm{H}_{38} \mathrm{NP}_{2}\right]^{+}: 450.2474$, found: 450.2472 . 


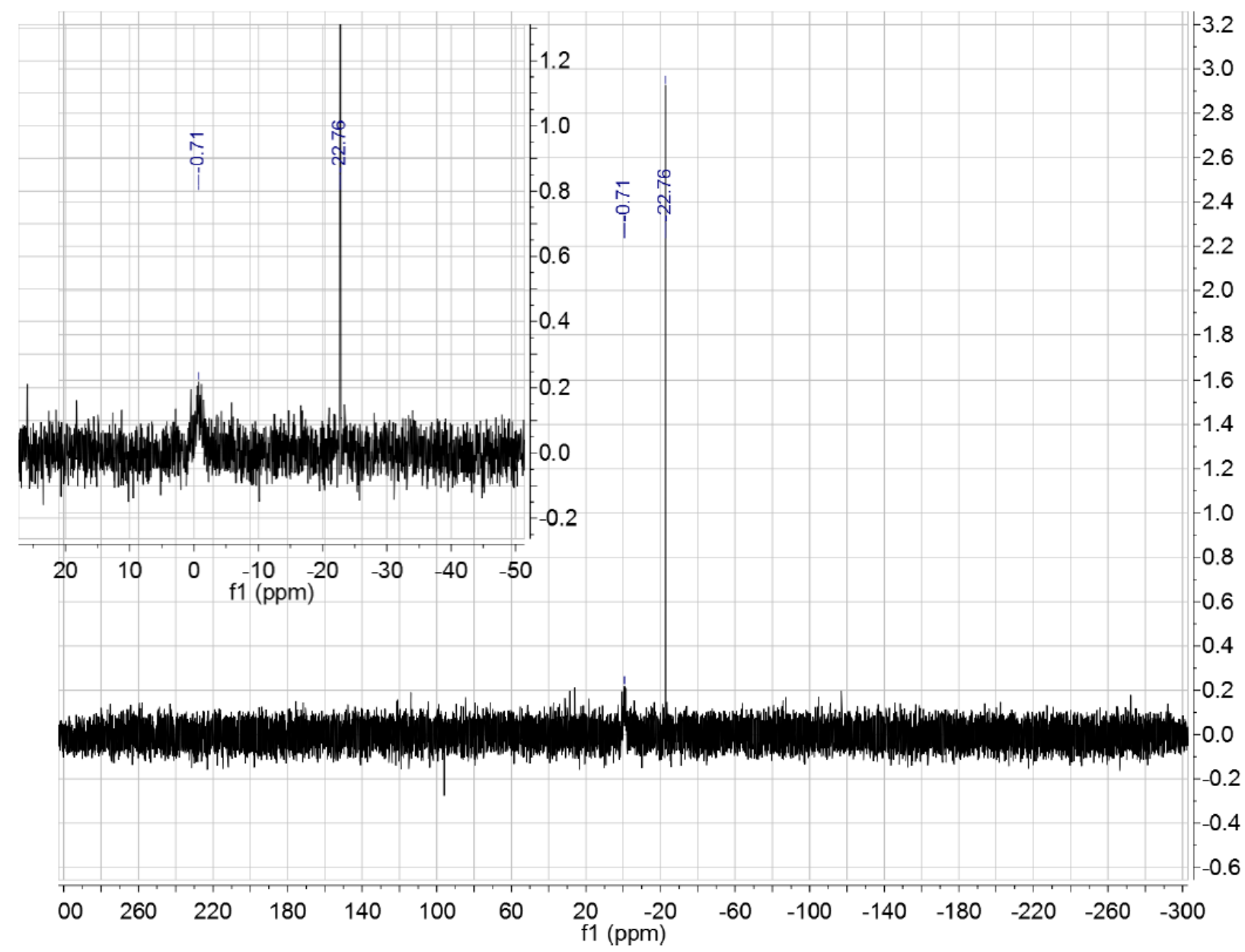

Figure S8: ${ }^{31} \mathrm{P}\left\{{ }^{1} \mathrm{H}\right\} \mathrm{NMR}$ spectrum $\left(242 \mathrm{MHz}, \mathrm{CD}_{2} \mathrm{Cl} 2\right)$ of $(\mathrm{S})-(\mathrm{iPr})_{2} \mathrm{PCH}_{2} \mathrm{CH}_{2} \mathrm{~N}(\mathrm{H}) \mathrm{CH}(\mathrm{Ph}) \mathrm{CH}_{2} \mathrm{P}(\mathrm{Ph})_{2} 3$ 


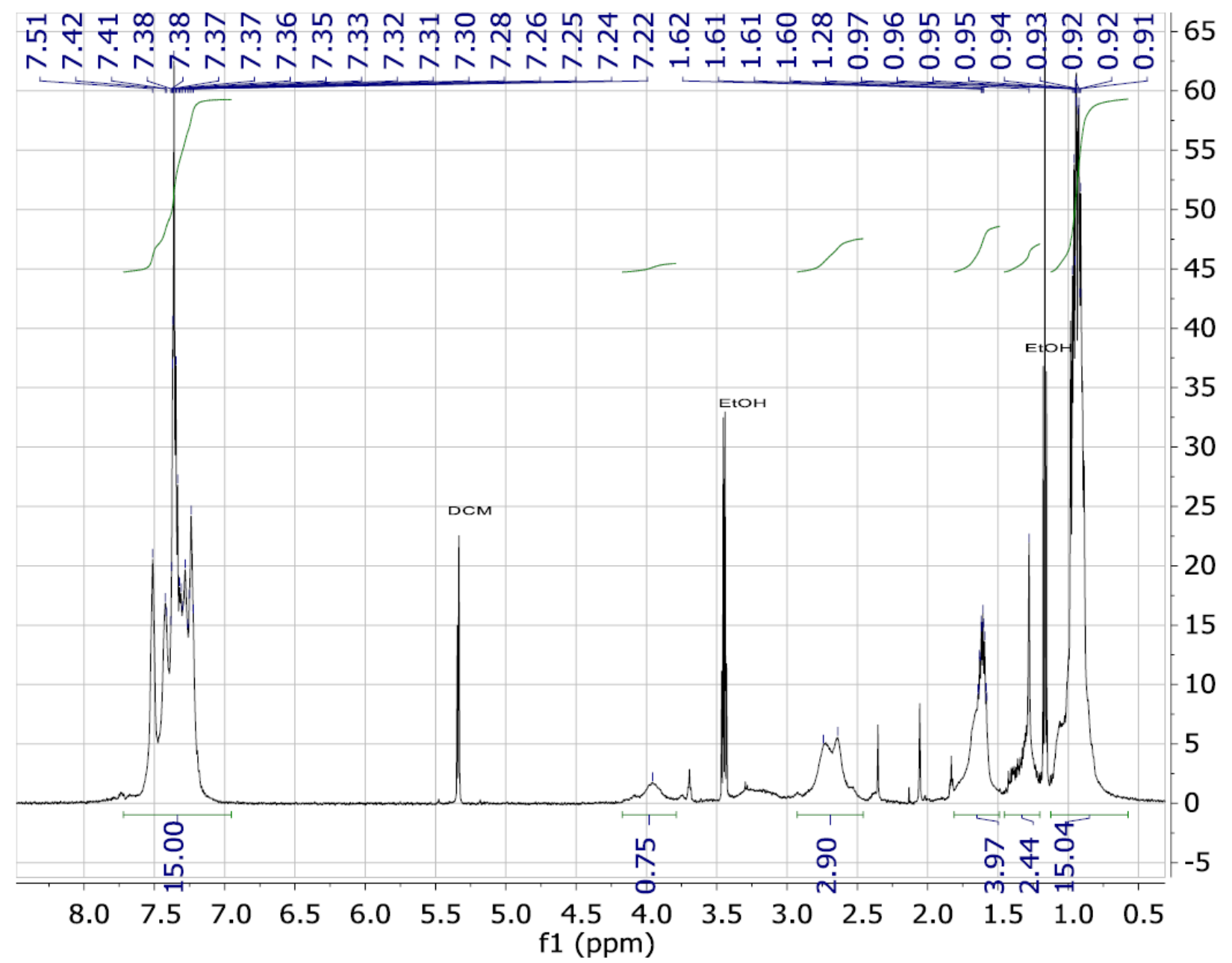

Figure S9: ${ }^{1} \mathrm{H}$ NMR spectrum $\left(600 \mathrm{MHz}, \mathrm{CD}_{2} \mathrm{Cl}_{2}\right)$ of $(\mathrm{S})-(\mathrm{iPr})_{2} \mathrm{PCH}_{2} \mathrm{CH}_{2} \mathrm{~N}(\mathrm{H}) \mathrm{CH}(\mathrm{Ph}) \mathrm{CH}_{2} \mathrm{P}(\mathrm{Ph})_{2} 3$.

\section{Synthesis of (S)-(iPr $)_{2} \mathrm{PCH}_{2} \mathrm{CH}_{2} \mathrm{~N}(\mathrm{H}) \mathrm{CH}\left(\mathrm{CH}\left(\mathrm{CH}_{3}\right)_{2}\right) \mathrm{CH}_{2} \mathrm{P}(\mathrm{Ph})_{2} 4$ :}

Follows the procedure above with (S)-1-(diphenylphosphino)-3-methyl-2-butylamine (300 mg, 1.11 mmol), iPr-phosphonium dimer (267 mg, $0.553 \mathrm{mmol})$, and trisacetoxy borohydride (469 mg, $2.21 \mathrm{mmol})$. This reaction yielded a colourless, viscous oil. Yield $320 \mathrm{mg}(70 \%) .{ }^{1} \mathrm{H}$ NMR $\left(500 \mathrm{MHz}, \mathrm{CD}_{2} \mathrm{Cl}_{2}\right)$ ס: 0.97-1.13 (m, $\left.18 \mathrm{H}, \mathrm{C}\left(\mathrm{CH}_{3}\right)_{2} \times 3\right), 1.73$ (sept, $\left.3 \mathrm{H}, \mathrm{CH}\left(\mathrm{CH}_{3}\right)_{2} \times 3\right), 1.86$ (bm, $\left.1 \mathrm{H}, \mathrm{CHPiPr} 2\right), 2.31$ (bm, 1H, CHPiPr $)_{2}, 2.47$ (dd, $1 \mathrm{H}, \mathrm{CH}(\mathrm{iPr})), 2.53\left(\mathrm{bm}, 1 \mathrm{H}, \mathrm{CHPPh}_{2}\right), 2.75$ (bm, $\left.1 \mathrm{H}, \mathrm{CHPPh}_{2}\right), 3.01$ (bm, $\left.1 \mathrm{H}, \mathrm{CHNH}\right), 3.15$ (bm, CHNH), 7.35-7.49 (m, $10 \mathrm{H}, \mathrm{Ar}-\mathrm{H}) .{ }^{13} \mathrm{C}\left\{{ }^{1} \mathrm{H}\right\} \mathrm{NMR}\left(\mathrm{CD}_{2} \mathrm{Cl}_{2}, 124 \mathrm{MHz}\right): 134.9\left(\mathrm{~d}, \mathrm{~J}_{\mathrm{CP}}=20 \mathrm{~Hz}, \mathrm{C}_{\mathrm{Ar}}\right), 134.6\left(\mathrm{~d},{ }^{2} \mathrm{~J}_{\mathrm{CP}}=19 \mathrm{~Hz}\right.$, $\left.C_{\mathrm{Ar}}\right), 132.9\left(\mathrm{D},{ }^{2} J_{\mathrm{CP}}=9 \mathrm{~Hz}, C_{\mathrm{Ar}}\right), 132.6\left(\mathrm{~d},{ }^{2} J_{\mathrm{CP}}=10 \mathrm{~Hz}, C_{\mathrm{Ar}}\right), 131.0\left(C_{\mathrm{Ar}}\right), 130.9\left(C_{\mathrm{Ar}}\right), 130.8\left(C_{\mathrm{Ar}}\right), 62.7\left(\mathrm{~d},{ }^{2} J_{\mathrm{CP}}=\right.$ $16 \mathrm{~Hz}, \mathrm{CHPPh}$ ) $, 48.6\left(\mathrm{~d},{ }^{2} \mathrm{~J}_{\mathrm{CP}}=35 \mathrm{~Hz}, \mathrm{CHNH}\right), 32.4\left(\mathrm{~d},{ }^{2} \mathrm{~J}_{\mathrm{CP}}=7 \mathrm{~Hz}, \mathrm{CH}(\mathrm{iPr})\right), 30.3(\mathrm{~m}, \mathrm{CHPiPr} 2), 25.1\left(\mathrm{dd},{ }^{2} \mathrm{JPP}_{\mathrm{CP}}=\right.$ $\left.12,8 \mathrm{~Hz}, C_{\mathrm{iPr}}\right), 21.7\left(\mathrm{dd},{ }^{2} J_{\mathrm{CP}}=16,2 \mathrm{~Hz}, C_{\mathrm{iPr}}\right), 20.6\left(\mathrm{~m}, C_{\mathrm{iPr}}\right), 20.4\left(\mathrm{~d}, J_{\mathrm{CP}}=9 \mathrm{~Hz}, C_{\mathrm{iPr}}\right), 19.4\left(\mathrm{~d}, J_{\mathrm{CP}}=54 \mathrm{~Hz}, C_{\mathrm{iPr}}\right)$. ${ }^{31} \mathrm{P}\left\{{ }^{1} \mathrm{H}\right\}$ NMR $\left(202 \mathrm{MHz}, \mathrm{CD}_{2} \mathrm{Cl}_{2}\right) \delta: 0.21\left(\mathrm{~s}, \mathrm{iPr}_{2} P\right),-21.98 \mathrm{ppm}\left(\mathrm{s}, \mathrm{Ph}_{2} P\right.$ ). HRMS (ESI-TOF, $\left.\mathrm{CH}_{2} \mathrm{Cl}_{2}\right) \mathrm{m} / \mathrm{z}$ calculated for $\left[\mathrm{C}_{25} \mathrm{H}_{40} \mathrm{NP}_{2}\right]^{+}: 416.2630$, found: 416.2628 . 


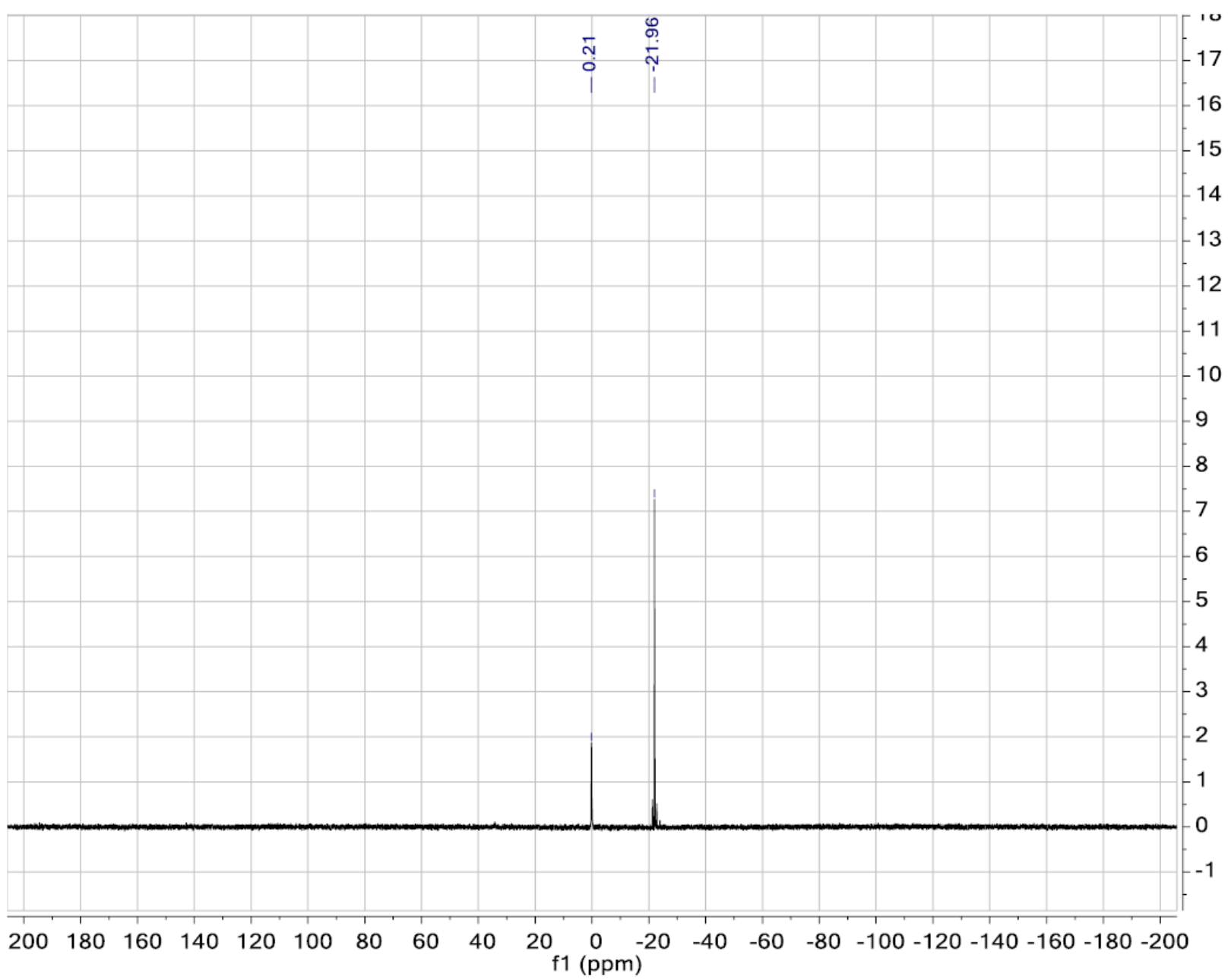

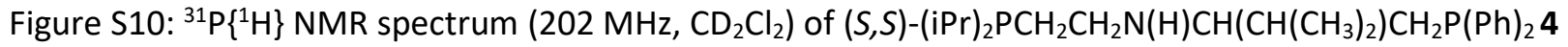




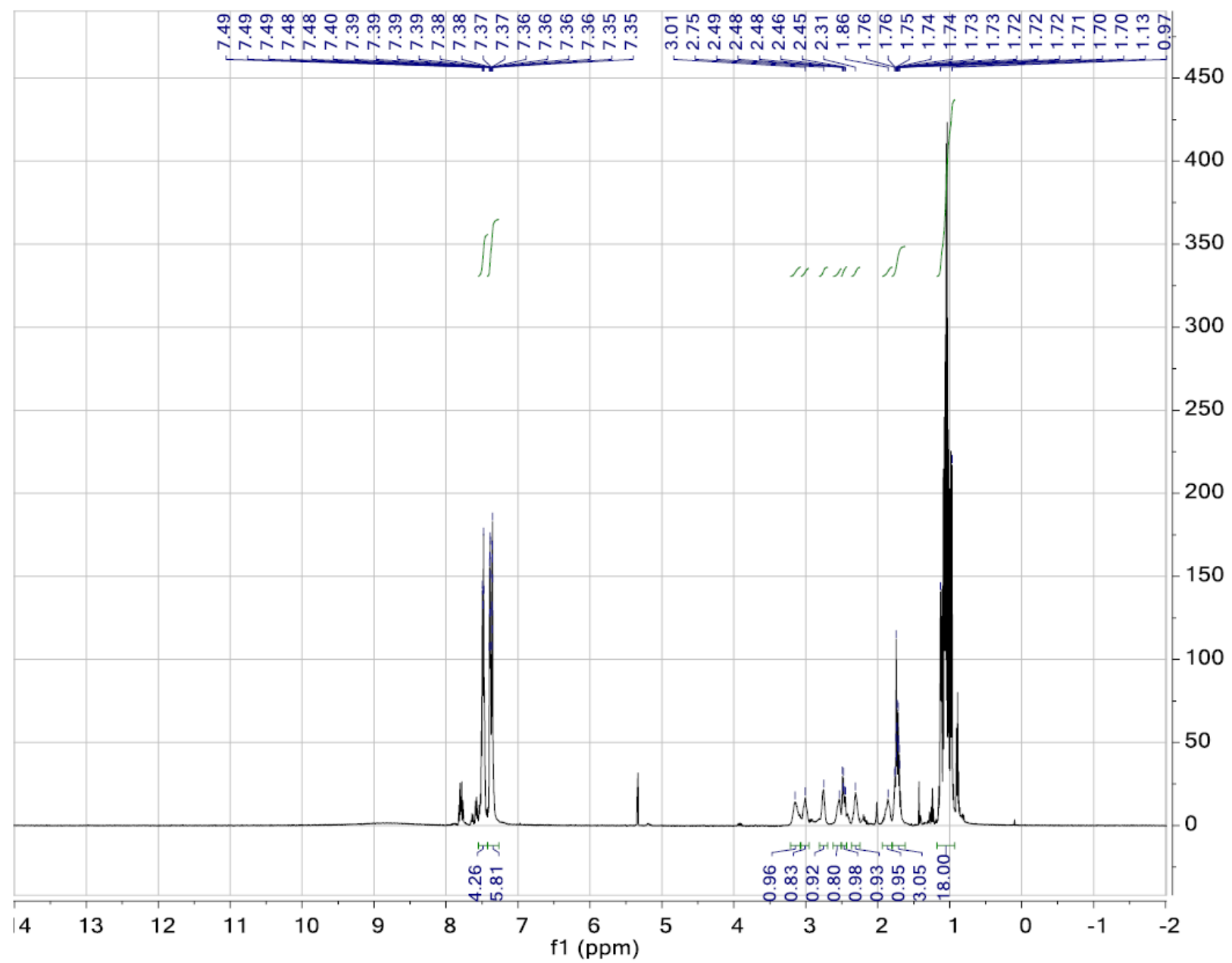

Figure S11: ${ }^{1} \mathrm{H}$ NMR spectrum $\left(500 \mathrm{MHz}, \mathrm{CD}_{2} \mathrm{Cl}\right.$ ) of $(\mathrm{S}, \mathrm{S})-(\mathrm{iPr})_{2} \mathrm{PCH}_{2} \mathrm{CH}_{2} \mathrm{~N}(\mathrm{H}) \mathrm{CH}\left(\mathrm{CH}\left(\mathrm{CH}_{3}\right)_{2}\right) \mathrm{CH}_{2} \mathrm{P}(\mathrm{Ph})_{2} 4$ 


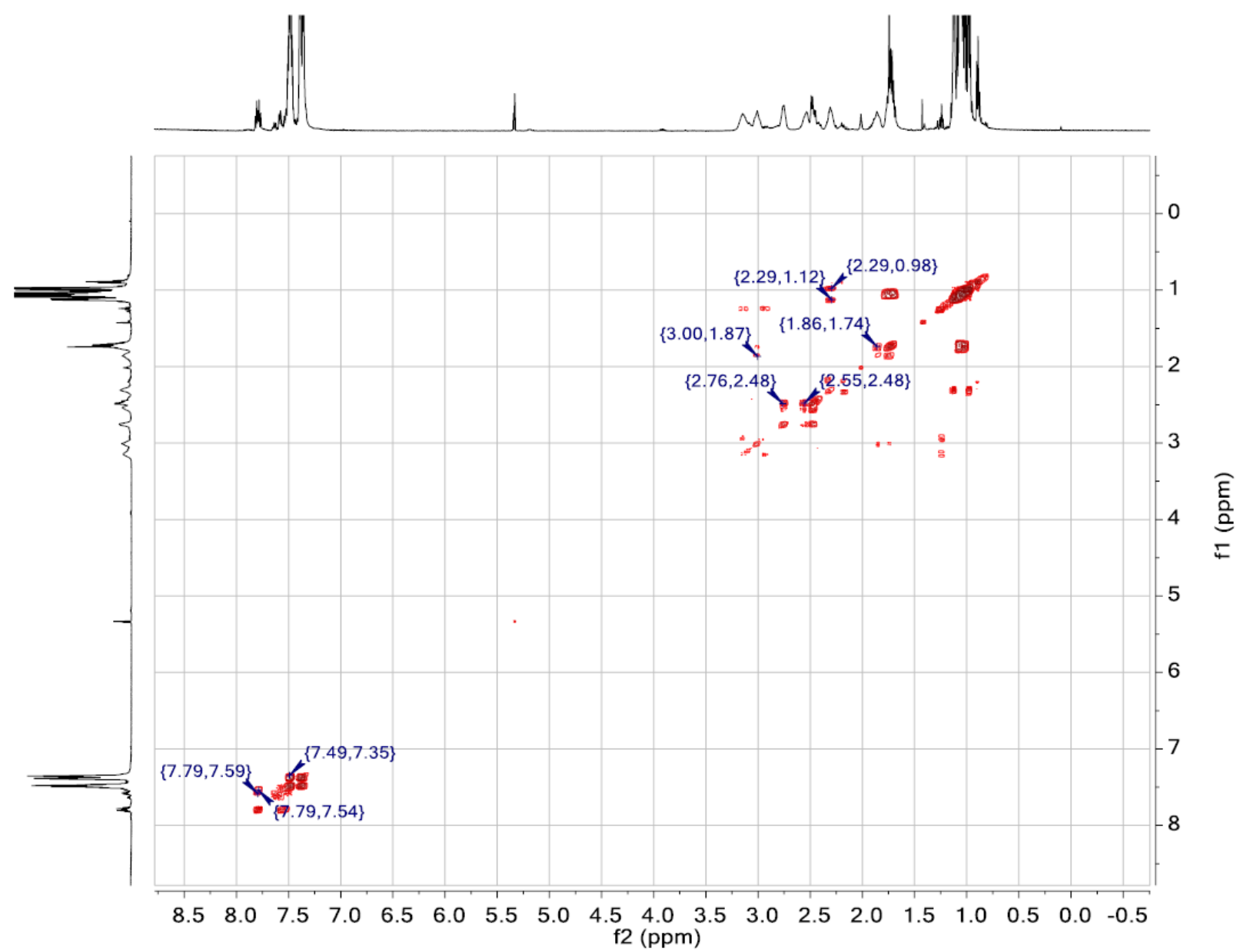

Figure S12: ${ }^{1} \mathrm{H}$-gCOSY $\left(500 \mathrm{MHz}, \mathrm{CD}_{2} \mathrm{Cl}_{2}\right)$ of $(\mathrm{S}, \mathrm{S})-(\mathrm{iPr})_{2} \mathrm{PCH}_{2} \mathrm{CH}_{2} \mathrm{~N}(\mathrm{H}) \mathrm{CH}\left(\mathrm{CH}\left(\mathrm{CH}_{3}\right)_{2}\right) \mathrm{CH}_{2} \mathrm{P}(\mathrm{Ph})_{2} 4$ 


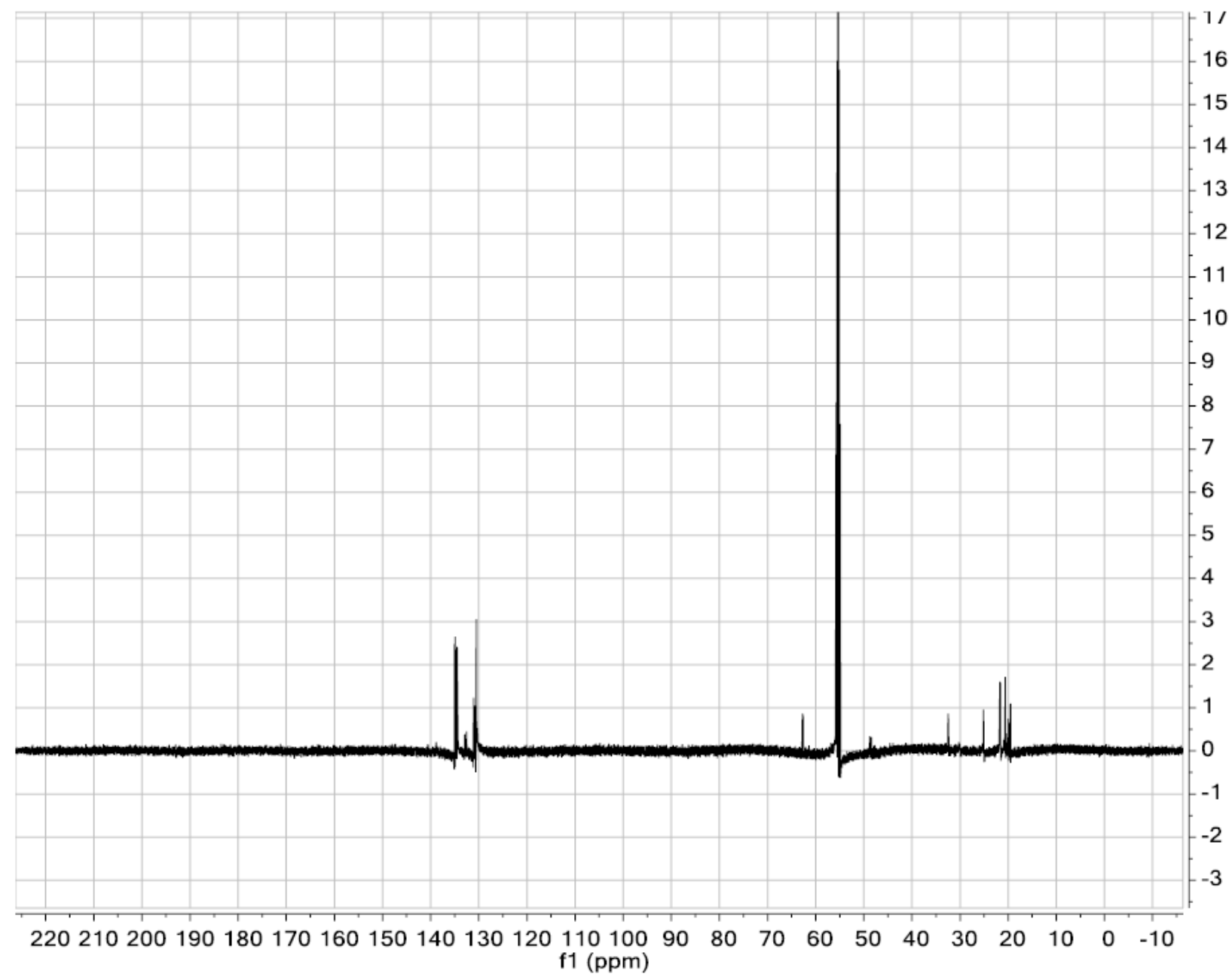

Figure S13: ${ }^{13} \mathrm{C}\left\{{ }^{1} \mathrm{H}\right\}\left(126 \mathrm{MHz}, \mathrm{CD}_{2} \mathrm{Cl}_{2}\right)$ of $(\mathrm{S}, \mathrm{S})-(\mathrm{PPr})_{2} \mathrm{PCH}_{2} \mathrm{CH}_{2} \mathrm{~N}(\mathrm{H}) \mathrm{CH}\left(\mathrm{CH}\left(\mathrm{CH}_{3}\right)_{2}\right) \mathrm{CH}_{2} \mathrm{P}(\mathrm{Ph})_{2} 4$

Synthesis of trans- $\mathrm{Fe}(\mathrm{Cl})_{2}(\mathrm{CO})[(\mathrm{S}, \mathrm{S})$ -

(iPr) $\left.{ }_{2} \mathrm{PCH}_{2} \mathrm{CH}_{2} \mathrm{~N}(\mathrm{H}) \mathrm{CH}(\mathrm{Ph}) \mathrm{CH}(\mathrm{Ph}) \mathrm{P}(\mathrm{Ph})_{2}\right](\mathrm{S}, \mathrm{S})-5$ :

A Schlenk flask was charged with $(\mathrm{S}, \mathrm{S})-(\mathrm{PPr})_{2} \mathrm{PCH}_{2} \mathrm{CH}{ }_{2} \mathrm{~N}(\mathrm{H}) \mathrm{CH}(\mathrm{Ph}) \mathrm{CH}(\mathrm{Ph}) \mathrm{P}(\mathrm{Ph})_{2}(80 \mathrm{mg}, 0.153 \mathrm{mmol}), \mathrm{FeCl}_{2}$ $(19 \mathrm{mg}, 0.153 \mathrm{mmol})$ and THF $(15 \mathrm{~mL})$. The flask was degassed and exposed to $\mathrm{CO}_{(\mathrm{g})}(1 \mathrm{~atm})$. The colourless slurry immediately turned dark purple. After $1 \mathrm{~h}$, the solvent was removed and the purple residue was dissolved with benzene, filtered and dried. (66 mg, $64 \%)$. ${ }^{1} \mathrm{H}$ NMR $\left(\mathrm{C}_{6} \mathrm{D}_{6}, 600 \mathrm{MHz}\right): 7.91(\mathrm{~m}, 2 \mathrm{H}, \mathrm{PArH})$, $7.80(\mathrm{~m}, 2 \mathrm{H}, \mathrm{PArH}), 7.48(\mathrm{~m}, 1 \mathrm{H}, \operatorname{ArH}), 7.15(\mathrm{~m}, 1 \mathrm{H}, \operatorname{ArH}), 7.02(\mathrm{~m}, 2 \mathrm{H}, \mathrm{PArH}), 6.89(\mathrm{~m}, 2 \mathrm{H}, \operatorname{ArH}), 6.81(\mathrm{~m}$, $3 \mathrm{H}, \operatorname{ArH}), 6.74(\mathrm{~m}, 2 \mathrm{H}, \mathrm{PArH}), 6.70(\mathrm{~m}, 1 \mathrm{H}, \operatorname{ArH}), 6.62(\mathrm{~m}, 2 \mathrm{H}, \operatorname{ArH}), 6.55(\mathrm{~m}, 1 \mathrm{H}, \operatorname{ArH}), 5.75(\mathrm{~m}, 1 \mathrm{H}, \mathrm{NH})$, $5.28\left(\mathrm{t}, J_{\mathrm{HH}}=12 \mathrm{~Hz}, 1 \mathrm{H}, \mathrm{NHCHPh}\right), 4.91\left(\mathrm{dd}, J_{\mathrm{HH}}=12 \mathrm{~Hz}, J_{\mathrm{HP}}=7 \mathrm{~Hz}, 1 \mathrm{H}, \mathrm{CH}(\mathrm{Ph}) \mathrm{PPh}_{2}\right), 3.13(\mathrm{~m}, 1 \mathrm{H}$, $\left.\mathrm{CH}_{2} \mathrm{CH}_{2} \mathrm{PiPr}_{2}\right), 2.87\left(\mathrm{dd}, J_{\mathrm{HH}}=12 \mathrm{~Hz}, J_{\mathrm{HP}}=35 \mathrm{~Hz}, 1 \mathrm{H}, \mathrm{CH}_{2} \mathrm{CH}_{2} \mathrm{PiPr}_{2}\right), 2.66\left(\mathrm{~m}, 2 \mathrm{H}, \mathrm{PCH}\left(\mathrm{CH}_{3}\right)_{2}\right), 1.77\left(\mathrm{t}, J_{\mathrm{HH}}=14\right.$ $\left.\mathrm{Hz}, 1 \mathrm{H}, \mathrm{CH}_{2} \mathrm{PiPr}_{2}\right), 1.67\left(\mathrm{~m}, J_{\mathrm{HH}}=14 \mathrm{~Hz}, 1 \mathrm{H}, \mathrm{CH}_{2} \mathrm{PiPr}_{2}\right), 1.49$ (dd, $\left.J_{\mathrm{HH}}=7 \mathrm{~Hz}, J_{\mathrm{HP}}=14 \mathrm{~Hz}, 3 \mathrm{H}, \mathrm{PCH}\left(\mathrm{CH}_{3}\right)_{2}\right), 1.38$ $\left(\mathrm{dd}, J_{\mathrm{HH}}=7 \mathrm{~Hz}, J_{\mathrm{HP}}=14 \mathrm{~Hz}, 3 \mathrm{H}, \mathrm{PCH}\left(\mathrm{CH}_{3}\right)_{2}\right), 1.28\left(\mathrm{dd}, J_{\mathrm{HH}}=7 \mathrm{~Hz}, J_{\mathrm{HP}}=12 \mathrm{~Hz}, 3 \mathrm{H}, \mathrm{PCH}\left(\mathrm{CH}_{3}\right)_{2}\right), 1.14\left(\mathrm{dd}, J_{\mathrm{HH}}=\right.$ $\left.7 \mathrm{~Hz}, J_{\mathrm{HP}}=12 \mathrm{~Hz}, 3 \mathrm{H}, \mathrm{PCH}\left(\mathrm{CH}_{3}\right)_{2}\right) .{ }^{13} \mathrm{C}\left\{{ }^{1} \mathrm{H}\right\} \operatorname{NMR}\left(\mathrm{C}_{6} \mathrm{D}_{6}, 124 \mathrm{MHz}\right): 223.9\left(\mathrm{t},{ }^{2} \mathrm{~J}_{\mathrm{CP}}=22 \mathrm{~Hz}, \mathrm{CO}\right), 138.1\left(\mathrm{~d}, J_{\mathrm{CP}}=\right.$ $\left.9 \mathrm{~Hz}, \mathrm{P} C_{\mathrm{Ar}}\right), 132.5\left(\mathrm{~d}, J_{\mathrm{CP}}=8 \mathrm{~Hz}, \mathrm{P} C_{\mathrm{Ar}}\right), 131.2\left(C_{\mathrm{Ar}}\right), 130.5\left(\mathrm{P} C_{\mathrm{Ar}}\right), 129.6\left(C_{\mathrm{Ar}}\right), 128.5\left(C_{\mathrm{Ar}}\right), 128.4\left(C_{\mathrm{Ar}}\right), 128.4$ $\left(C_{\mathrm{Ar}}\right), 127.9\left(C_{\mathrm{Ar}}\right), 127.6\left(\mathrm{P} C_{\mathrm{Ar}}\right), 126.8\left(\mathrm{~d}, J_{\mathrm{CP}}=9 \mathrm{~Hz}, \mathrm{P} C_{\mathrm{Ar}}\right), 126.4\left(C_{\mathrm{Ar}}\right), 123.4\left(C_{\mathrm{Ar}}\right), 71.3\left(\mathrm{dd}, J_{\mathrm{CP}}=3,13 \mathrm{~Hz}\right.$, 
CHPh), 55.7 (d, $\left.J_{\mathrm{CP}}=5 \mathrm{~Hz}, \mathrm{CH}(\mathrm{Ph}) \mathrm{PPh}_{2}\right), 47.6\left(\mathrm{~m}, \mathrm{CH}_{2} \mathrm{CH}_{2} \mathrm{PiPr}_{2}\right), 26.2\left(\mathrm{~d}, J_{\mathrm{CP}}=12 \mathrm{~Hz}, \mathrm{CH}_{2} \mathrm{PiPr}_{2}\right), 23.3\left(\mathrm{~d}, J_{\mathrm{CP}}=\right.$ $\left.21 \mathrm{~Hz}, \mathrm{CH}\left(\mathrm{CH}_{3}\right)_{2}\right), 21.2\left(\mathrm{~d}, J_{\mathrm{CP}}=17 \mathrm{~Hz}, \mathrm{CH}\left(\mathrm{CH}_{3}\right)_{2}\right), 20.2\left(\mathrm{CH}_{3}\right), 19.5\left(\mathrm{CH}_{3}\right), 18.9\left(\mathrm{CH}_{3}\right), 18.3\left(\mathrm{CH}_{3}\right) .{ }^{31} \mathrm{P}\left\{{ }^{1} \mathrm{H}\right\} \mathrm{NMR}$ $\left(\mathrm{C}_{6} \mathrm{D}_{6}, 242 \mathrm{MHz}\right): 71.1$ (d, $\left.{ }^{2} J_{\mathrm{PP}}=177 \mathrm{~Hz}, \mathrm{PiPr}_{2}\right), 69.5$ (d, $\left.{ }^{2} \mathrm{JPP}_{\mathrm{PP}}=177 \mathrm{~Hz}, P \mathrm{Ph}_{2}\right)$ ppm. EA: Calc. for $\mathrm{C}_{35} \mathrm{H}_{41} \mathrm{Cl}_{2} \mathrm{FeNOP}_{2} .0 .5 \mathrm{C}_{6} \mathrm{H}_{6}$ : Expected C, 63.4; $\mathrm{H}, 6.2 ; \mathrm{N}, 1.9$. Found: $\mathrm{C}, 63.6, \mathrm{H}, 5.6, \mathrm{~N}, 1.8$. AT-IR $1940 \mathrm{~cm}^{-1}$ $v(\mathrm{CO})$.

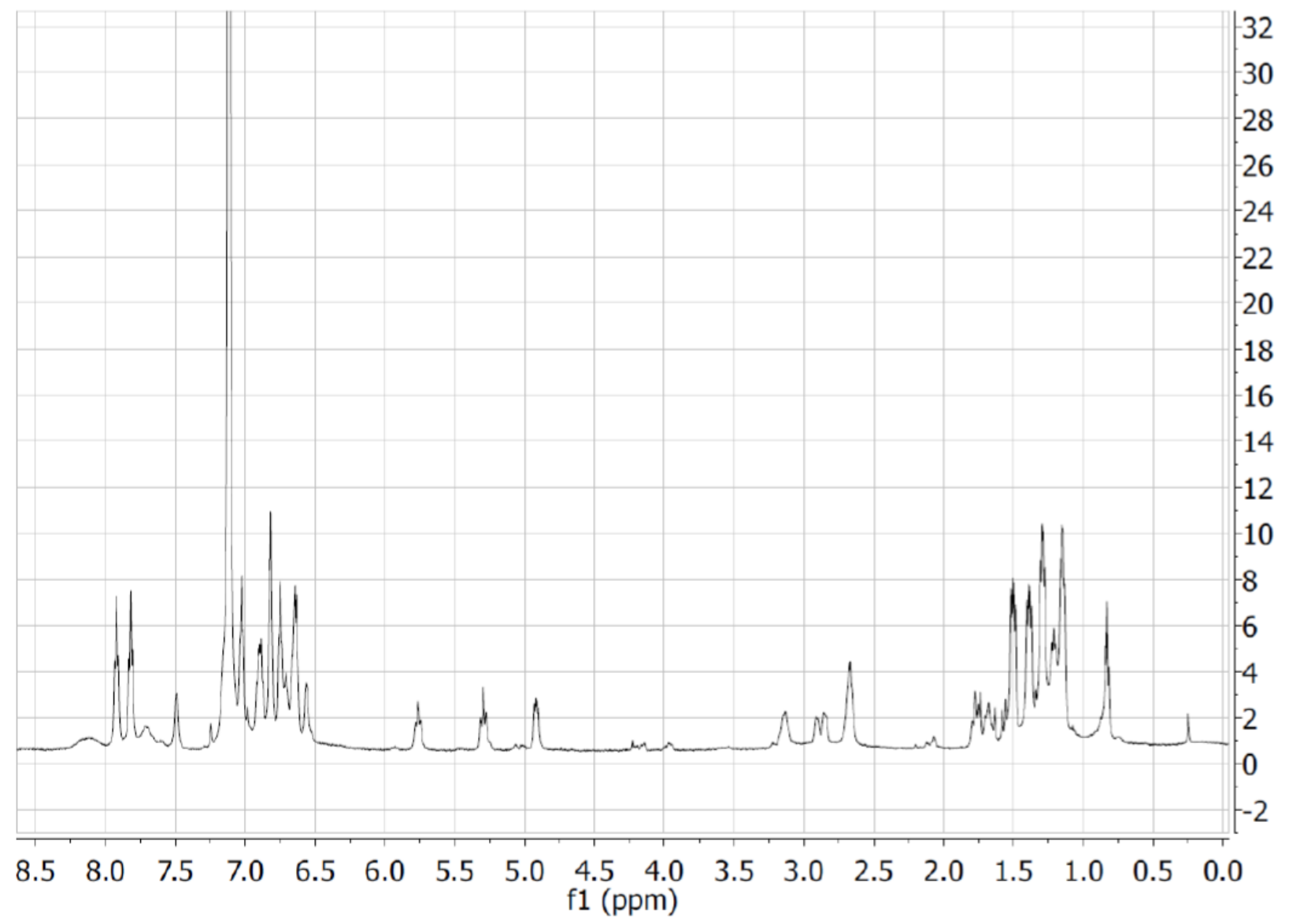

Figure $\quad \mathrm{S} 14: \quad{ }^{1} \mathrm{H} \quad \mathrm{NMR} \quad$ spectrum $\quad\left(600 \quad \mathrm{MHz}, \quad \mathrm{C}_{6} \mathrm{D}_{6}\right)$ of trans- $\mathrm{Fe}(\mathrm{Cl})_{2}(\mathrm{CO})[(\mathrm{S}, \mathrm{S})$ (iPr) $\left.{ }_{2} \mathrm{PCH}_{2} \mathrm{CH}_{2} \mathrm{~N}(\mathrm{H}) \mathrm{CH}(\mathrm{Ph}) \mathrm{CH}(\mathrm{Ph}) \mathrm{P}(\mathrm{Ph})_{2}\right](\mathrm{S}, \mathrm{S})-5$. 


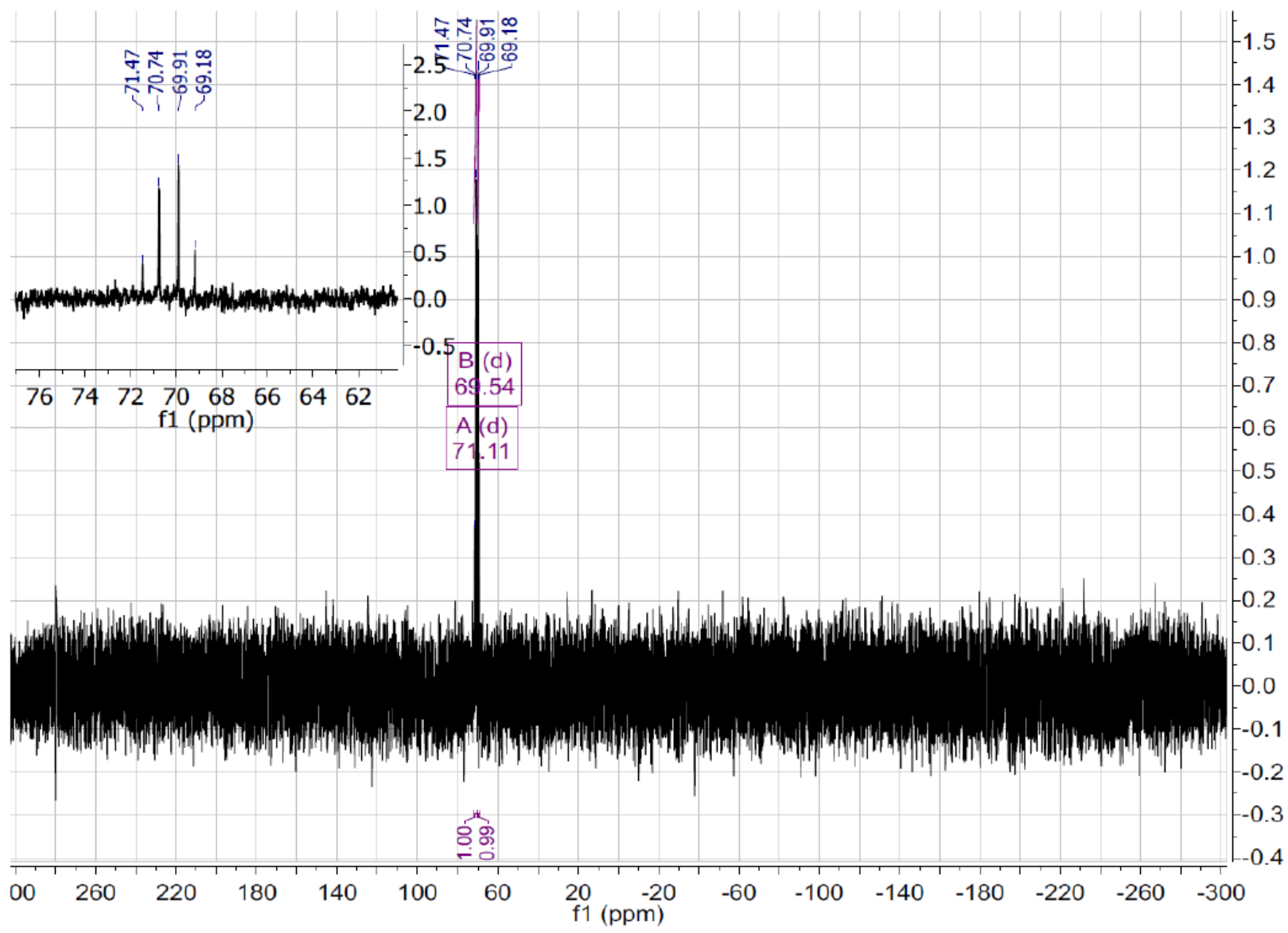

Figure $\quad \mathrm{S} 15: \quad{ }^{31} \mathrm{P}\left\{{ }^{1} \mathrm{H}\right\} \quad \mathrm{NMR}$ spectrum $\left(242 \quad \mathrm{MHz}, \quad \mathrm{C}_{6} \mathrm{D}_{6}\right)$ of trans- $\mathrm{Fe}(\mathrm{Cl})_{2}(\mathrm{CO})[(\mathrm{S}, \mathrm{S})$ (iPr) $\left.{ }_{2} \mathrm{PCH}_{2} \mathrm{CH}_{2} \mathrm{~N}(\mathrm{H}) \mathrm{CH}(\mathrm{Ph}) \mathrm{CH}(\mathrm{Ph}) \mathrm{P}(\mathrm{Ph})_{2}\right](\mathrm{S}, \mathrm{S})-5$. 


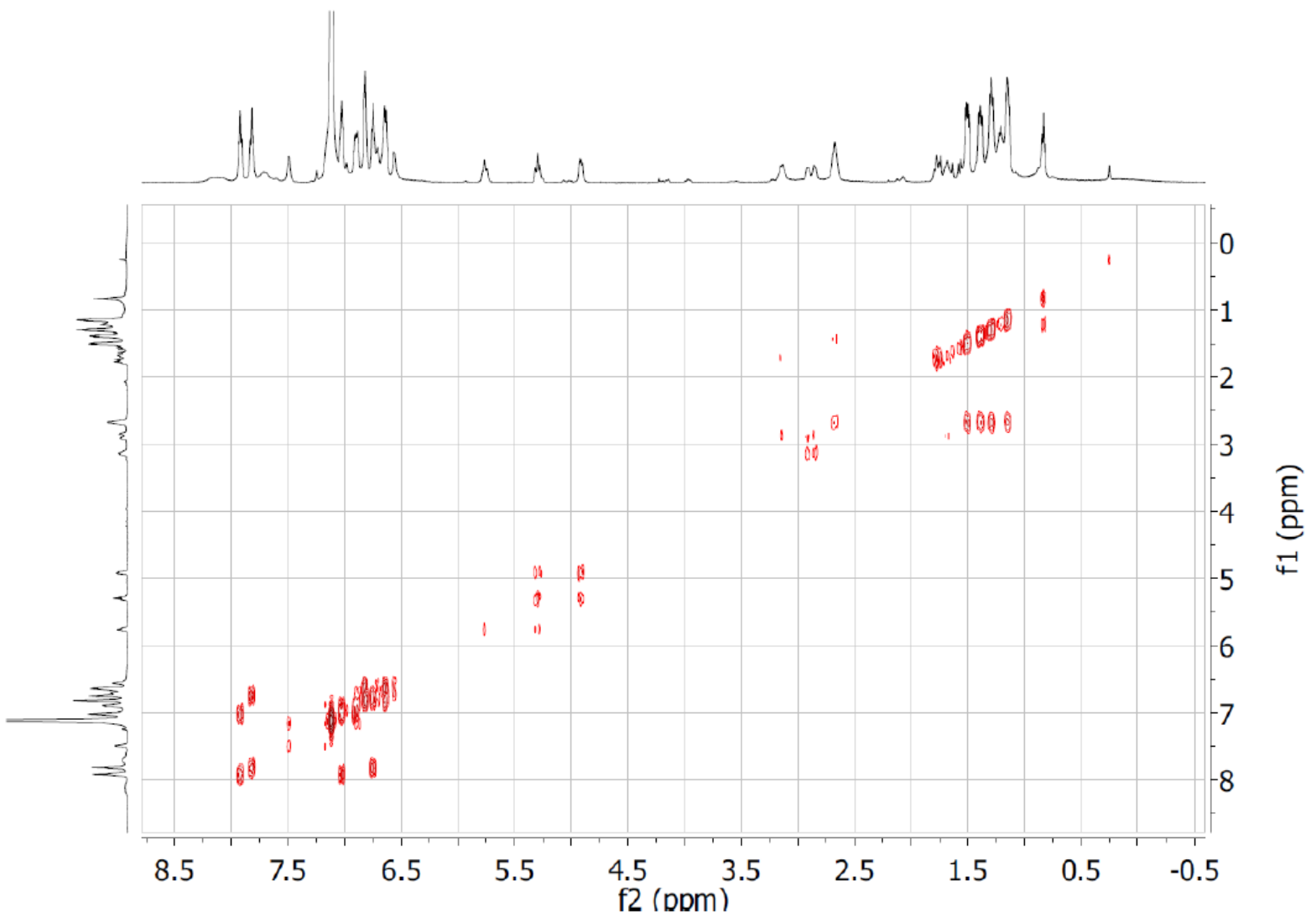

Figure S16: ${ }^{1} \mathrm{H} \quad \mathrm{COSY} \quad \mathrm{NMR}$ spectrum $\left(600 \quad \mathrm{MHz}, \quad \mathrm{C}_{6} \mathrm{D}_{6}\right)$ of trans-Fe $(\mathrm{Cl})_{2}(\mathrm{CO})[(\mathrm{S}, \mathrm{S})$ (iPr) $\left.{ }_{2} \mathrm{PCH}_{2} \mathrm{CH}_{2} \mathrm{~N}(\mathrm{H}) \mathrm{CH}(\mathrm{Ph}) \mathrm{CH}(\mathrm{Ph}) \mathrm{P}(\mathrm{Ph})_{2}\right](\mathrm{S}, \mathrm{S})-5$. 


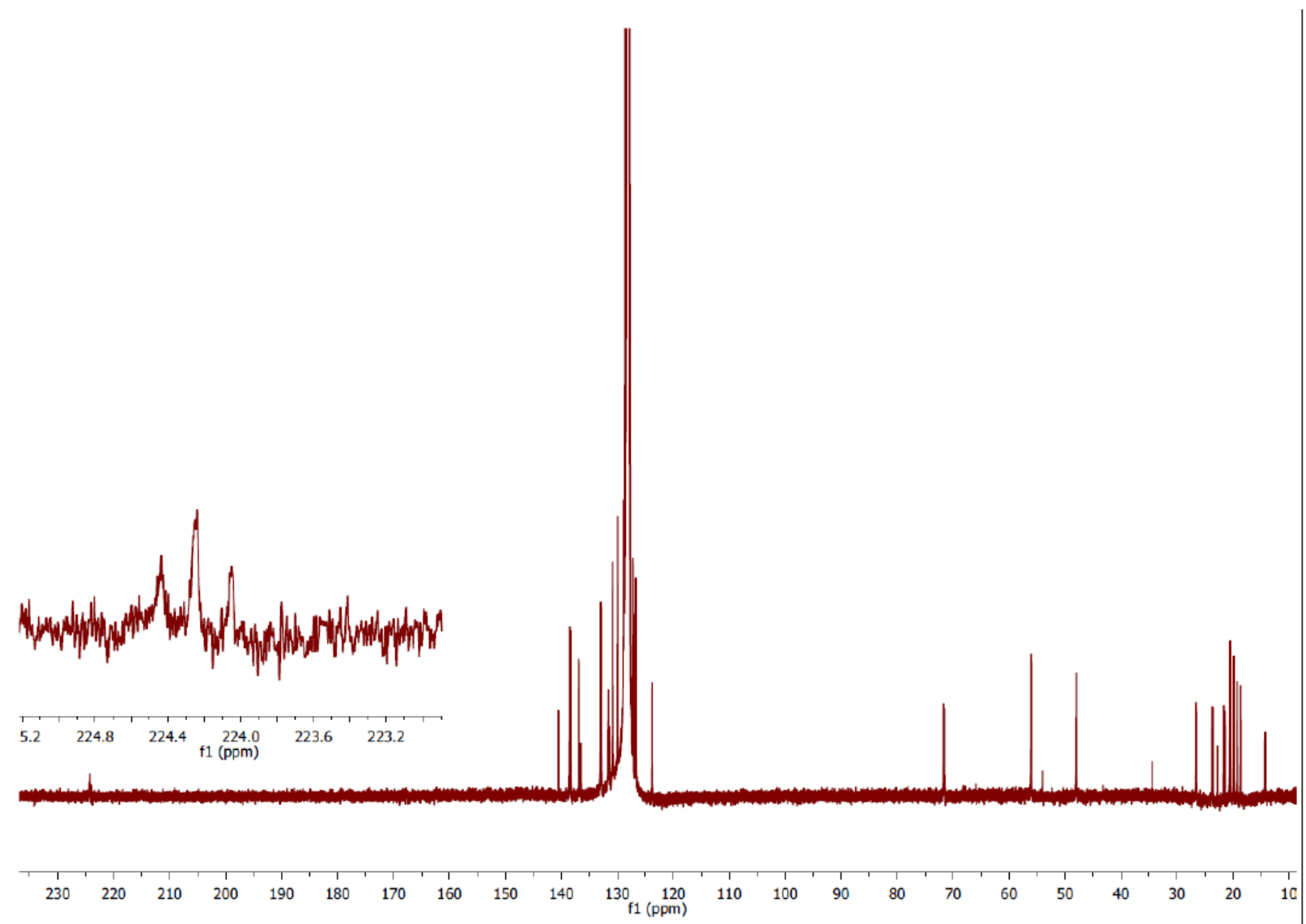

Figure $\quad \mathrm{S} 17: \quad{ }^{13} \mathrm{C}\left\{{ }^{1} \mathrm{H}\right\} \quad \mathrm{NMR}$ spectrum $\left(124 \quad \mathrm{MHz}, \quad \mathrm{C}_{6} \mathrm{D}_{6}\right)$ of trans- $\mathrm{Fe}(\mathrm{Cl})_{2}(\mathrm{CO})[(\mathrm{S}, \mathrm{S})$ (iPr) $\left.{ }_{2} \mathrm{PCH}_{2} \mathrm{CH}_{2} \mathrm{~N}(\mathrm{H}) \mathrm{CH}(\mathrm{Ph}) \mathrm{CH}(\mathrm{Ph}) \mathrm{P}(\mathrm{Ph})_{2}\right](S, S)-5$.

Synthesis of trans- $\mathrm{Fe}(\mathrm{Cl})_{2}(\mathrm{CO})[(\mathrm{S}, \mathrm{S})$ $\left.(\mathrm{Cy})_{2} \mathrm{PCH}_{2} \mathrm{CH}_{2} \mathrm{~N}(\mathrm{H}) \mathrm{CH}(\mathrm{Ph}) \mathrm{CH}(\mathrm{Ph}) \mathrm{P}(\mathrm{Ph})_{2}\right](\mathrm{S}, \mathrm{S})-6$ :

The procedure above was followed with $(\mathrm{S}, \mathrm{S})-(\mathrm{Cy})_{2} \mathrm{PCH}_{2} \mathrm{CH}_{2} \mathrm{~N}(\mathrm{H}) \mathrm{CH}(\mathrm{Ph}) \mathrm{CH}(\mathrm{Ph}) \mathrm{P}(\mathrm{Ph})_{2}(216 \mathrm{mg}, 0.357 \mathrm{mmol})$ and $\mathrm{FeCl}_{2}$ (33 mg, $\left.0.206 \mathrm{mmol}\right)$. Yielded a purple solid $(203 \mathrm{mg}, 74 \%) .{ }^{1} \mathrm{H} \mathrm{NMR}\left(\mathrm{CD}_{2} \mathrm{Cl}_{2}, 600 \mathrm{MHz}\right): 7.86$ $6.79(\mathrm{~m}, 2 \mathrm{H}, \mathrm{PArH}), 5.54\left(\mathrm{td}, \mathrm{J}_{\mathrm{HH}}=12,4 \mathrm{~Hz}, 1 \mathrm{H}, \mathrm{NH}\right), 5.13\left(\mathrm{td}, J_{\mathrm{HH}}=12,3 \mathrm{~Hz}, 1 \mathrm{H}, \mathrm{NHCH}(\mathrm{Ph})\right), 4.86\left(\mathrm{dd}, J_{\mathrm{HH}}=\right.$ $\left.12 \mathrm{~Hz}, J_{\mathrm{HH}}=7 \mathrm{~Hz}, 1 \mathrm{H}, \mathrm{CH}(\mathrm{Ph}) \mathrm{PPh}_{2}\right), 3.21-3.06\left(\mathrm{~m}, 2 \mathrm{H}, \mathrm{CH}_{2} \mathrm{CH}_{2} \mathrm{PCy}\right.$ ), 2.43 (q, JH $=12,8 \mathrm{~Hz}, 2 \mathrm{H}, \mathrm{CH}_{2} \mathrm{CH}_{2} \mathrm{PiPr}_{2}$ ), 2.28-1.12 (m, 22H, PC $\left.{ }_{6} H_{11}\right) \cdot{ }^{31} \mathrm{P}\left\{{ }^{1} \mathrm{H}\right\} \operatorname{NMR}\left(\mathrm{CD}_{2} \mathrm{Cl}_{2}, 242 \mathrm{MHz}\right): 70.1\left(\mathrm{~d},{ }^{2} J_{\mathrm{PP}}=173 \mathrm{~Hz}, \mathrm{PiPr}_{2}\right), 61.5\left(\mathrm{~d},{ }^{2} J_{\mathrm{PP}}=173\right.$ $\left.\mathrm{Hz}, P P h_{2}\right)$ ppm. AT-IR $1946 \mathrm{~cm}^{-1} v_{(\mathrm{CO})}$. EA Calc. for $\mathrm{C}_{41} \mathrm{H}_{49} \mathrm{Cl}_{2} \mathrm{FeNOP}_{2} .0 .7 \mathrm{C}_{6} \mathrm{H}_{6}: \mathrm{C}, 66.59 ; \mathrm{H}, 6.59 ; \mathrm{N}, 1.72$. Found: C, 66.88; H, 6.65; N, 1.74. 


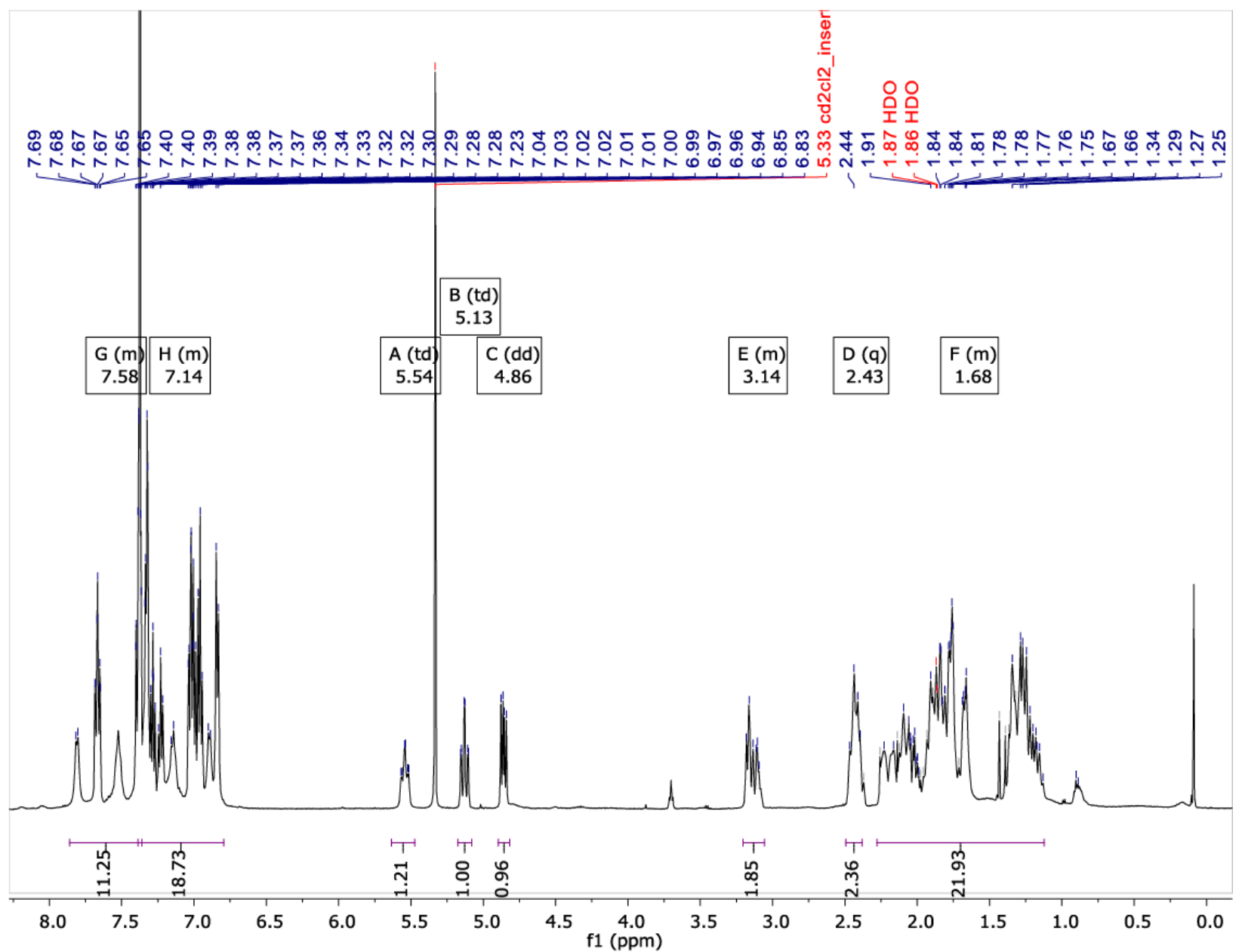

Figure S18: ${ }^{1} \mathrm{H} \quad \mathrm{NMR}$ spectrum $\left(500 \quad \mathrm{MHz}, \quad \mathrm{CD}_{2} \mathrm{Cl}_{2}\right)$ of trans- $\mathrm{Fe}(\mathrm{Cl})_{2}(\mathrm{CO})[(\mathrm{S}, \mathrm{S})$ $\left.(\mathrm{Cy})_{2} \mathrm{PCH}_{2} \mathrm{CH}_{2} \mathrm{~N}(\mathrm{H}) \mathrm{CH}(\mathrm{Ph}) \mathrm{CH}(\mathrm{Ph}) \mathrm{P}(\mathrm{Ph})_{2}\right](\mathrm{S}, \mathrm{S})-6$. 


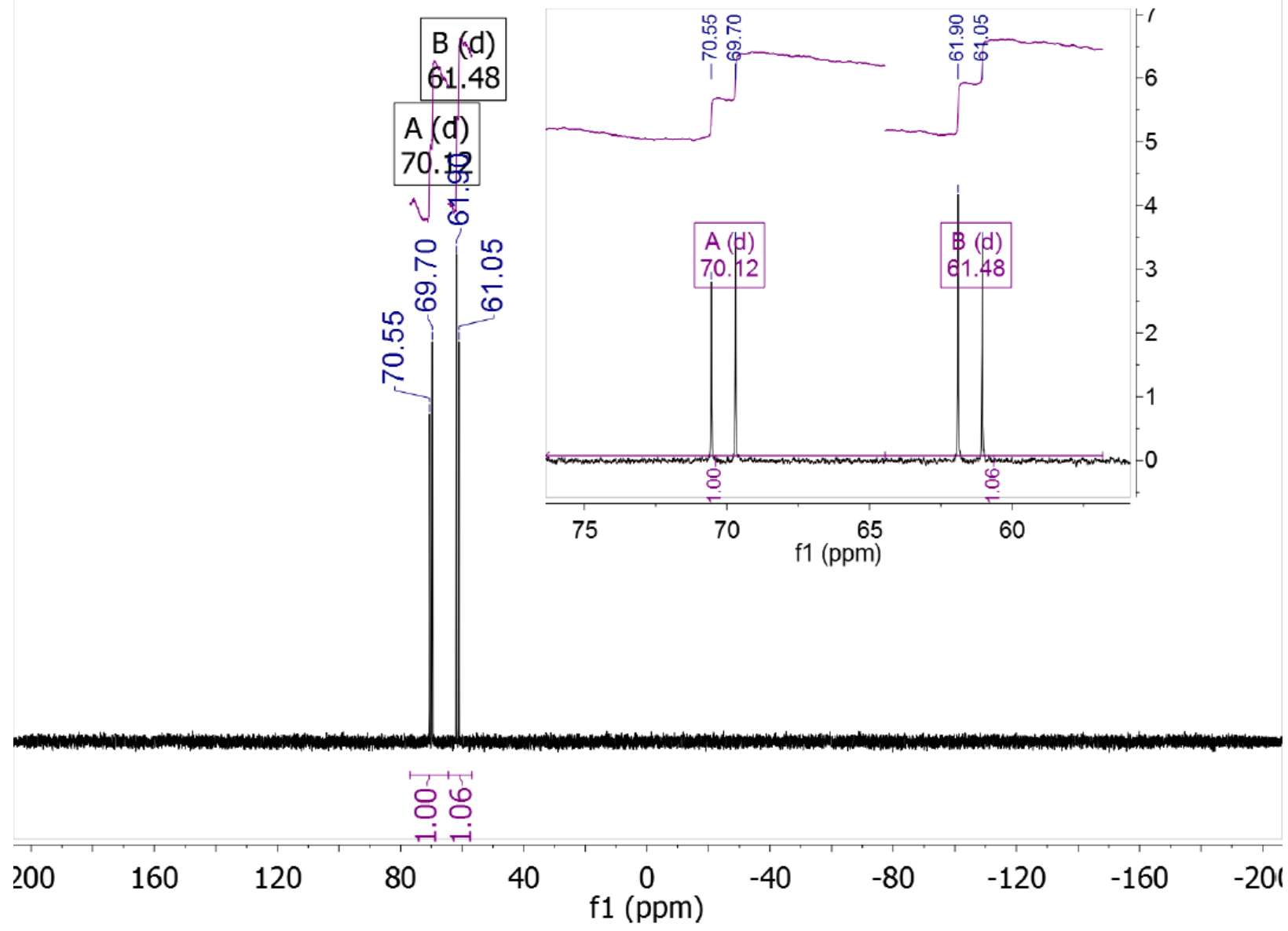

Figure S19: ${ }^{31} \mathrm{P}\left\{{ }^{1} \mathrm{H}\right\} \quad \mathrm{NMR}$ spectrum $\left(202 \mathrm{MHz}, \mathrm{CD}_{2} \mathrm{Cl}_{2}\right)$ of trans-Fe(Cl)$)_{2}(\mathrm{CO})[(\mathrm{S}, \mathrm{S})$ $\left.(\mathrm{Cy})_{2} \mathrm{PCH}_{2} \mathrm{CH}_{2} \mathrm{~N}(\mathrm{H}) \mathrm{CH}(\mathrm{Ph}) \mathrm{CH}(\mathrm{Ph}) \mathrm{P}(\mathrm{Ph})_{2}\right](S, S)-6$. 


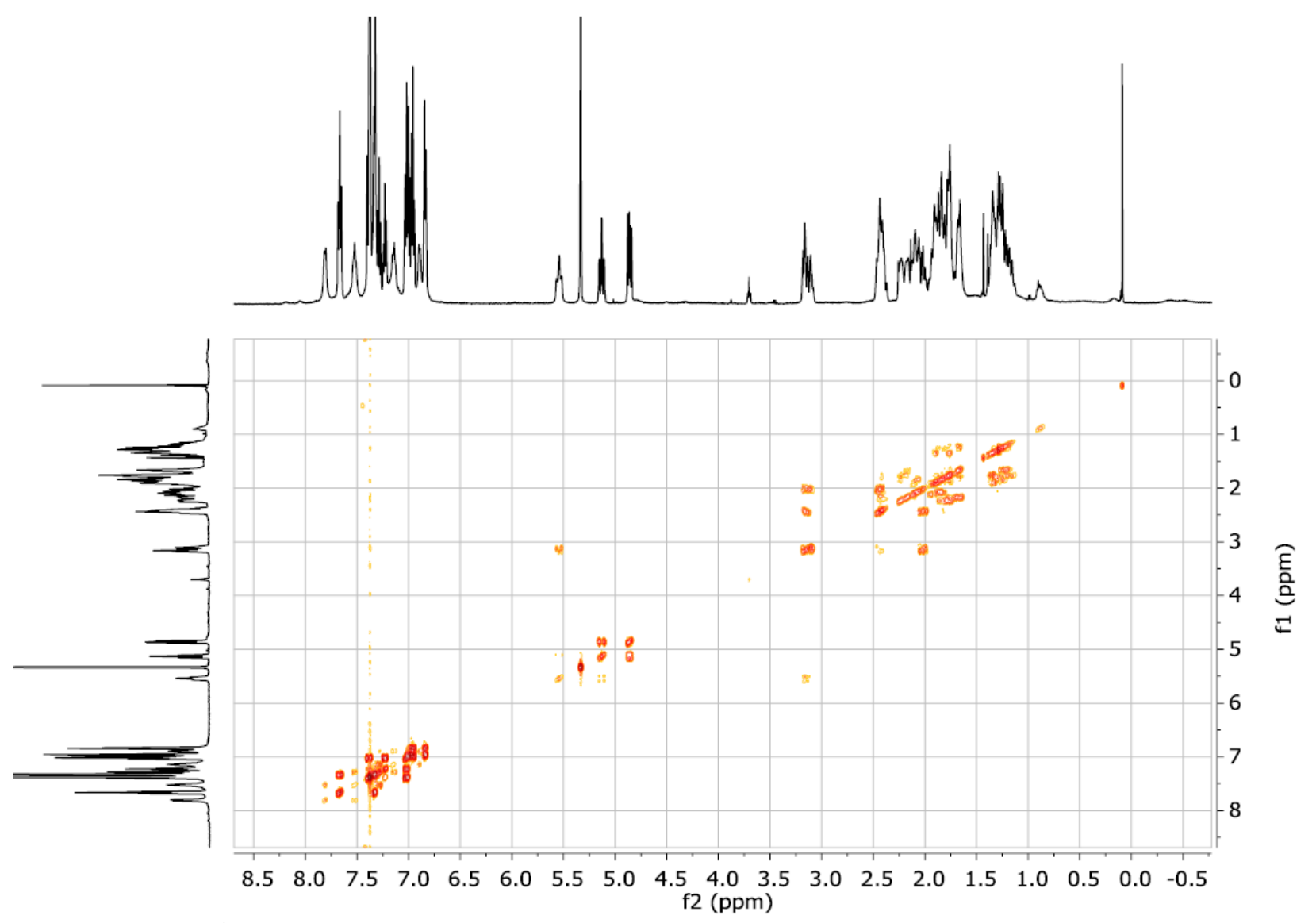

Figure $\mathrm{S} 20:{ }^{1} \mathrm{H} \quad \mathrm{COSY} \quad \mathrm{NMR}$ spectrum $\left(600 \quad \mathrm{MHz}, \quad \mathrm{CD}_{2} \mathrm{Cl}_{2}\right)$ of trans- $\mathrm{Fe}(\mathrm{Cl})_{2}(\mathrm{CO})[(\mathrm{S}, \mathrm{S})$ $\left.(\mathrm{Cy})_{2} \mathrm{PCH}_{2} \mathrm{CH}_{2} \mathrm{~N}(\mathrm{H}) \mathrm{CH}(\mathrm{Ph}) \mathrm{CH}(\mathrm{Ph}) \mathrm{P}(\mathrm{Ph})_{2}\right](\mathrm{S}, \mathbf{S})-6$.

\section{Synthesis of trans-Fe(Cl) $)_{2}(\mathrm{CO})\left[(\mathrm{S})-(\mathrm{iPr})_{2} \mathrm{PCH}_{2} \mathrm{CH}_{2} \mathrm{~N}(\mathrm{H}) \mathrm{CH}(\mathrm{Ph}) \mathrm{CH}_{2} \mathrm{P}(\mathrm{Ph})_{2}\right]$} (S)-7:

A Schlenk flask was charged with (S)-(iPr) ${ }_{2} \mathrm{PCH}_{2} \mathrm{CH}_{2} \mathrm{~N}(\mathrm{H}) \mathrm{CH}(\mathrm{Ph}) \mathrm{CH}_{2} \mathrm{P}(\mathrm{Ph})_{2}$ (50 mg, $\left.0.114 \mathrm{mmol}\right), \mathrm{FeCl}_{2}$ (15 $\mathrm{mg}, 0.114 \mathrm{mmol}$ ) and THF (15 mL). The flask was degassed and exposed to CO (1 atm). The colourless slurry immediately turned dark purple. After $1 \mathrm{~h}$, the solvent was removed and the purple residue was dissolved with benzene, filtered and dried. (40 mg, 60\%). Crystals suitable for X-ray crystallography were obtained from a slow diffusion of pentane into benzene. ${ }^{1} \mathrm{H} N M R\left(\mathrm{C}_{6} \mathrm{D}_{6}, 500 \mathrm{MHz}\right): 8.03(\mathrm{~m}, 2 \mathrm{H}, \mathrm{PArH})$, $7.79(\mathrm{~m}, 2 \mathrm{H}, \mathrm{PArH}), 7.05(\mathrm{~m}, 6 \mathrm{H}, \mathrm{PArH}), 6.97(\mathrm{~m}, 3 \mathrm{H}, \mathrm{ArH}), 5.30(\mathrm{~m}, 1 \mathrm{H}, \mathrm{NH}), 4.73(\mathrm{~m}, 1 \mathrm{H}, \mathrm{CHPh}), 3.05(\mathrm{~m}$, $\left.3 \mathrm{H}, \mathrm{CH}_{2} \mathrm{CH}_{2} \mathrm{PiPr}_{2}, \mathrm{CH}_{2} \mathrm{PPh}_{2}\right), 2.72\left(\mathrm{~m}, 2 \mathrm{H}, \mathrm{CH}\left(\mathrm{CH}_{3}\right)_{2}, \mathrm{CH}_{2} \mathrm{CH}_{2} \mathrm{PiPr}_{2}\right), 2.51\left(\mathrm{~m}, 1 \mathrm{H}, \mathrm{CH}\left(\mathrm{CH}_{3}\right)_{2}\right), 1.75\left(\mathrm{~m}, J_{\mathrm{HH}}=14\right.$ $\left.\mathrm{Hz}, 1 \mathrm{H}, \mathrm{CH}_{2} \mathrm{PiPr}_{2}\right), 1.55\left(\mathrm{dd}, J_{\mathrm{HH}}=7 \mathrm{~Hz}, J_{\mathrm{HP}}=15 \mathrm{~Hz}, 3 \mathrm{H}, \mathrm{CH}_{3}\right), 1.39$ (dd, $J_{\mathrm{HH}}=7 \mathrm{~Hz}, J_{\mathrm{HP}}=15 \mathrm{~Hz}, 4 \mathrm{H}, \mathrm{CH}_{3}$, $\left.\mathrm{CH}_{2} \mathrm{PiPr}_{2}\right), 1.31\left(\mathrm{dd}, J_{\mathrm{HH}}=7 \mathrm{~Hz}, J_{\mathrm{HP}}=15 \mathrm{~Hz}, 3 \mathrm{H}, \mathrm{CH}_{3}\right), 1.14\left(\mathrm{dd}, J_{\mathrm{HH}}=7 \mathrm{~Hz}, J_{\mathrm{HP}}=15 \mathrm{~Hz}, 3 \mathrm{H}, \mathrm{CH}_{3}\right) .{ }^{13} \mathrm{C}\left\{{ }^{1} \mathrm{H}\right\} \mathrm{NMR}$ $\left(C_{6} \mathrm{D}_{6}, 124 \mathrm{MHz}\right): 224.8\left(\mathrm{t},{ }^{2} J_{\mathrm{CP}}=25 \mathrm{~Hz}, \mathrm{CO}\right), 134.0\left(\mathrm{~d}, J_{\mathrm{CP}}=9 \mathrm{~Hz}, \mathrm{PC} C_{\mathrm{Ar}}\right), 132.2\left(\mathrm{~d}, J_{\mathrm{CP}}=8 \mathrm{~Hz}, \mathrm{PC} C_{\mathrm{Ar}}\right), 129.9$ (s, $\left.C_{\mathrm{Ar}}\right), 129.2\left(\mathrm{~s}, C_{\mathrm{Ar}}\right), 127.9\left(\mathrm{PC} \mathrm{Ar}_{\mathrm{Arr}} \mathrm{C}_{\mathrm{Ar}}\right), 64.3\left(\mathrm{dd}, J_{\mathrm{CP}}=3,8 \mathrm{~Hz}, \mathrm{CHPh}\right), 47.1\left(\mathrm{~m}, \mathrm{CH}_{2} \mathrm{CH}_{2} \mathrm{PiPr}_{2}\right), 37.3\left(\mathrm{~d}, J_{\mathrm{CP}}=15\right.$ $\left.\mathrm{Hz}, \mathrm{CH}_{2} \mathrm{PPh}_{2}\right), 26.7\left(\mathrm{~d}, J_{\mathrm{CP}}=13 \mathrm{~Hz}, \mathrm{CH}_{2} \mathrm{PiPr}_{2}\right), 23.9\left(\mathrm{~d}, J_{\mathrm{CP}}=22 \mathrm{~Hz}, \mathrm{CH}\left(\mathrm{CH}_{3}\right)_{2}\right), 22.5\left(\mathrm{~d}, \mathrm{~J}_{\mathrm{CP}}=19 \mathrm{~Hz}, \mathrm{CH}\left(\mathrm{CH}_{3}\right)_{2}\right)$, $20.3\left(\mathrm{~s}, \mathrm{CH}_{3}\right), 19.9\left(\mathrm{~s}, \mathrm{CH}_{3}\right), 19.2\left(\mathrm{~s}, \mathrm{CH}_{3}\right), 18.6\left(\mathrm{~s}, \mathrm{CH}_{3}\right) .{ }^{31} \mathrm{P}\left\{{ }^{1} \mathrm{H}\right\} \mathrm{NMR}\left(\mathrm{C}_{6} \mathrm{D}_{6}, 202 \mathrm{MHz}\right): 71.2\left(\mathrm{~d},{ }^{2} J_{\mathrm{PP}}=177 \mathrm{~Hz}\right.$, $\left.\mathrm{PiPr}_{2}\right), 52.8\left(\mathrm{~d},{ }^{2} \mathrm{JPP}_{\mathrm{PP}}=177 \mathrm{~Hz}, \mathrm{PPh}_{2}\right) \mathrm{ppm}$. AT-IR $1941 \mathrm{~cm}^{-1} \mathrm{v}_{(\mathrm{CO})}$. EA: Expected $57.64 \mathrm{C} \%, 6.17 \mathrm{H} \%, 2.32 \mathrm{~N} \%$. Found: 57.50 C\%, 5.89 H\%, 2.04 N\% 


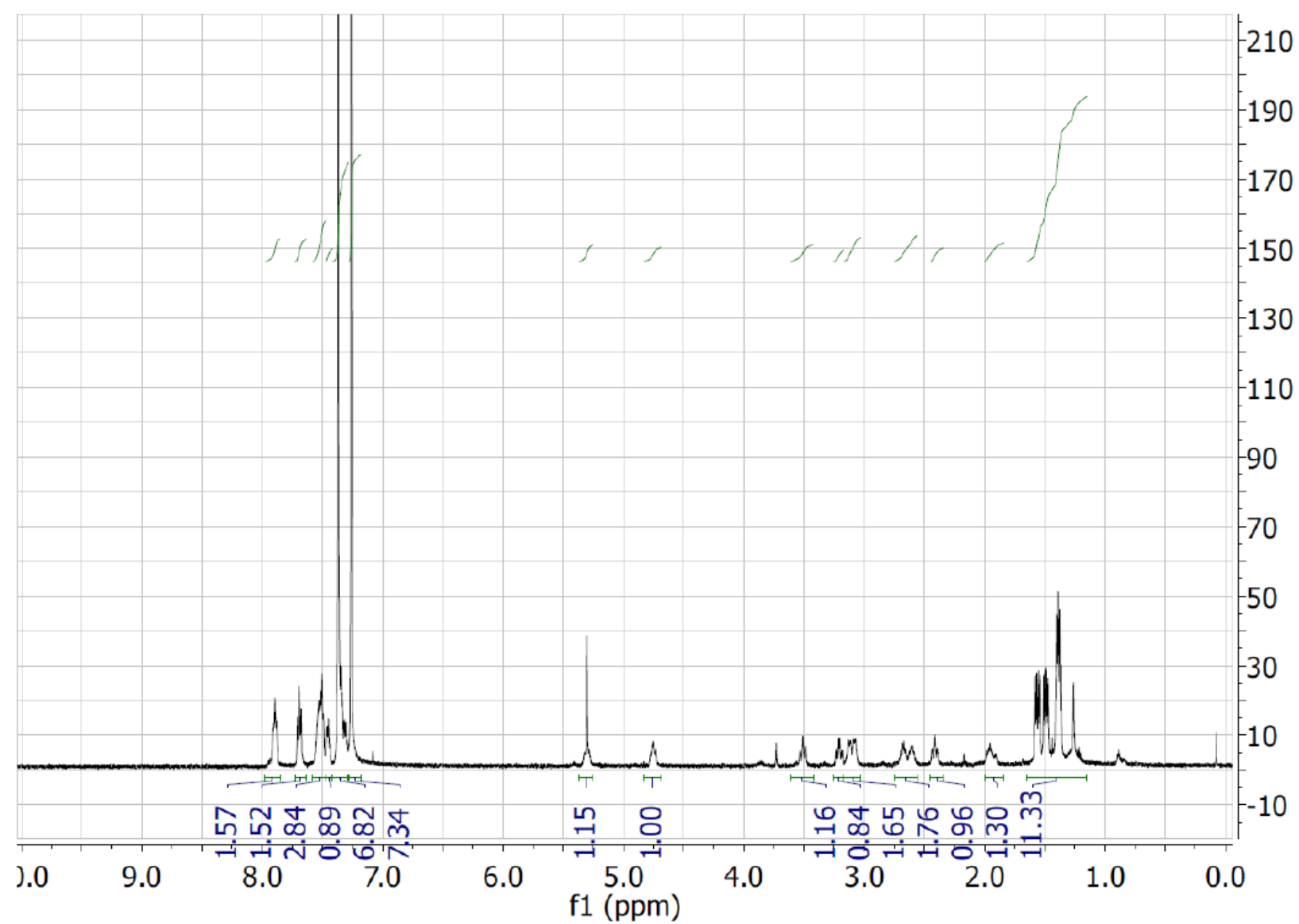

Figure $\mathrm{S} 21:{ }^{1} \mathrm{H}$ NMR spectrum $\left(500 \mathrm{MHz}, \mathrm{C}_{6} \mathrm{D}_{6}\right)$ of trans-Fe $(\mathrm{Cl})_{2}(\mathrm{CO})\left[(\mathrm{S})-(\mathrm{iPr})_{2} \mathrm{PCH}_{2} \mathrm{CH}{ }_{2} \mathrm{~N}(\mathrm{H}) \mathrm{CH}(\mathrm{Ph}) \mathrm{CH}_{2} \mathrm{P}(\mathrm{Ph})_{2}\right]$ $(S)-7$. 


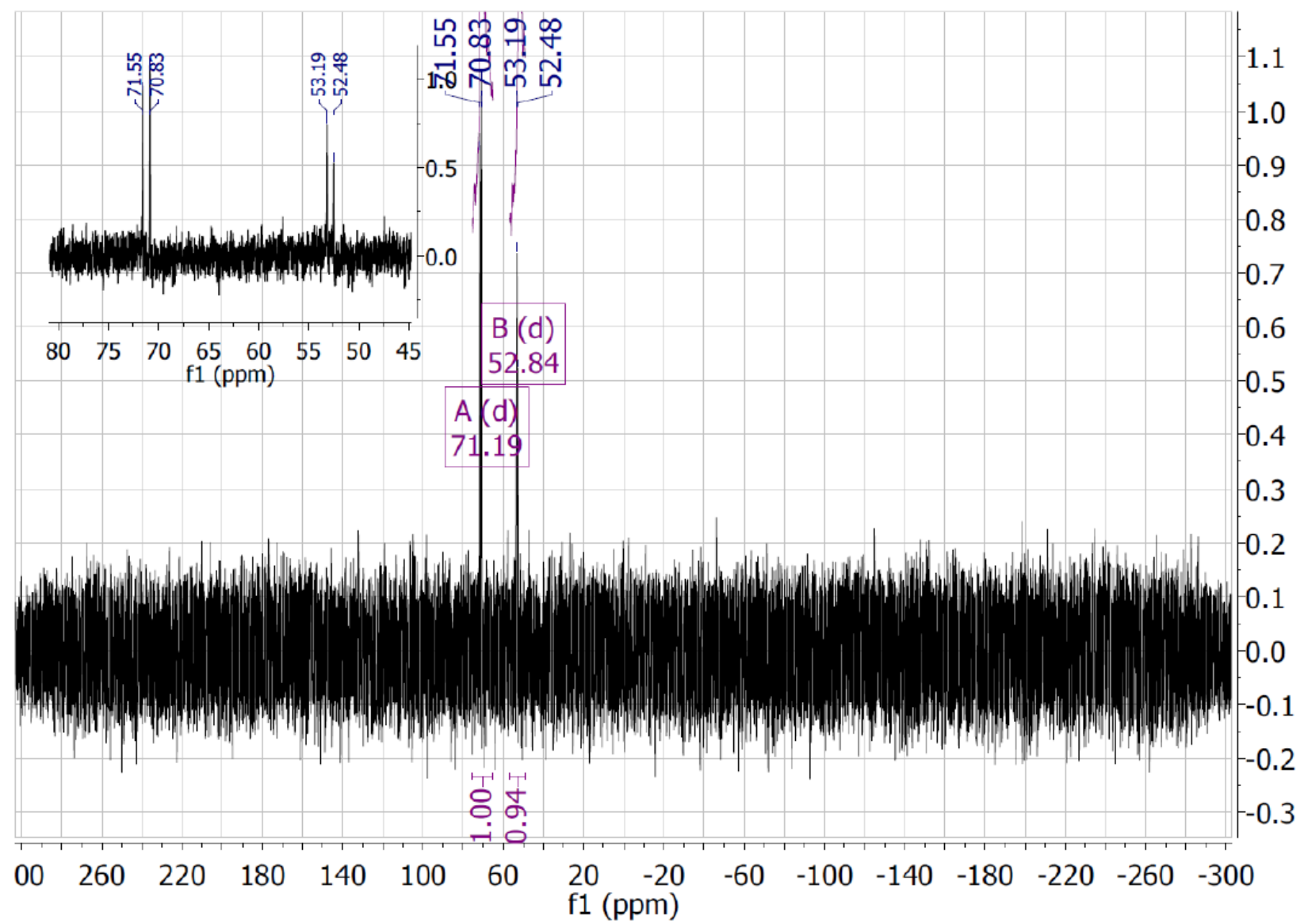

Figure $\quad \mathrm{S} 22: \quad{ }^{31} \mathrm{P}\left\{{ }^{1} \mathrm{H}\right\} \quad \mathrm{NMR} \quad$ spectrum $\quad\left(202 \quad \mathrm{MHz}, \quad \mathrm{C}_{6} \mathrm{D}_{6}\right) \quad$ of trans- $\mathrm{Fe}(\mathrm{Cl})_{2}(\mathrm{CO})[(\mathrm{S})$ (iPr) $\left.{ }_{2} \mathrm{PCH}_{2} \mathrm{CH}_{2} \mathrm{~N}(\mathrm{H}) \mathrm{CH}(\mathrm{Ph}) \mathrm{CH}_{2} \mathrm{P}(\mathrm{Ph})_{2}\right](\mathrm{S})-7$. 


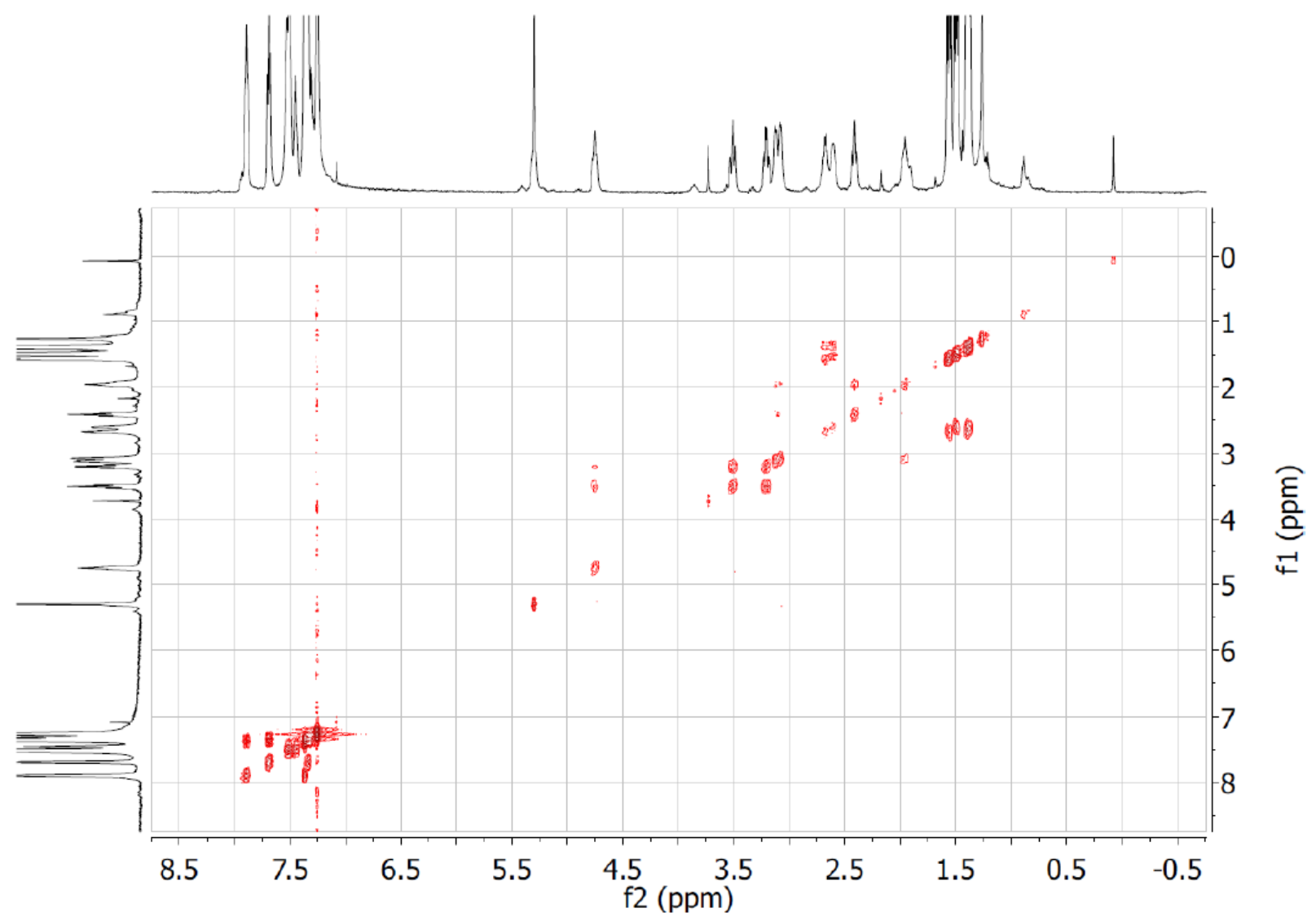

Figure S23: ${ }^{1} \mathrm{H} \quad \mathrm{COSY} \quad \mathrm{NMR}$ spectrum $\left(500 \quad \mathrm{MHz}, \mathrm{C}_{6} \mathrm{D}_{6}\right)$ of trans-Fe(Cl) ${ }_{2}(\mathrm{CO})[(\mathrm{S})$ (iPr) $\left.{ }_{2} \mathrm{PCH}_{2} \mathrm{CH}_{2} \mathrm{~N}(\mathrm{H}) \mathrm{CH}(\mathrm{Ph}) \mathrm{CH}_{2} \mathrm{P}(\mathrm{Ph})_{2}\right](\mathrm{S})-7$. 


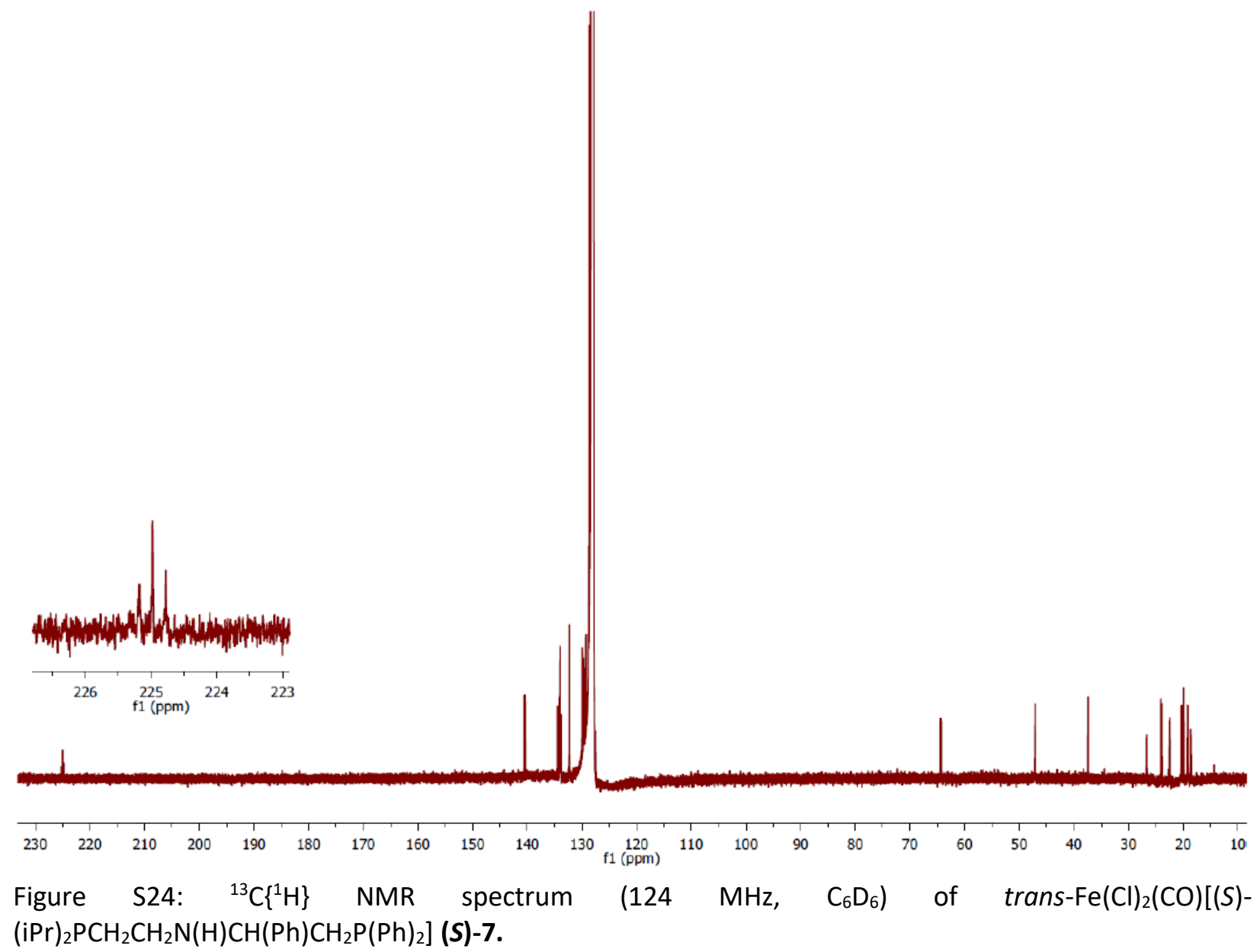




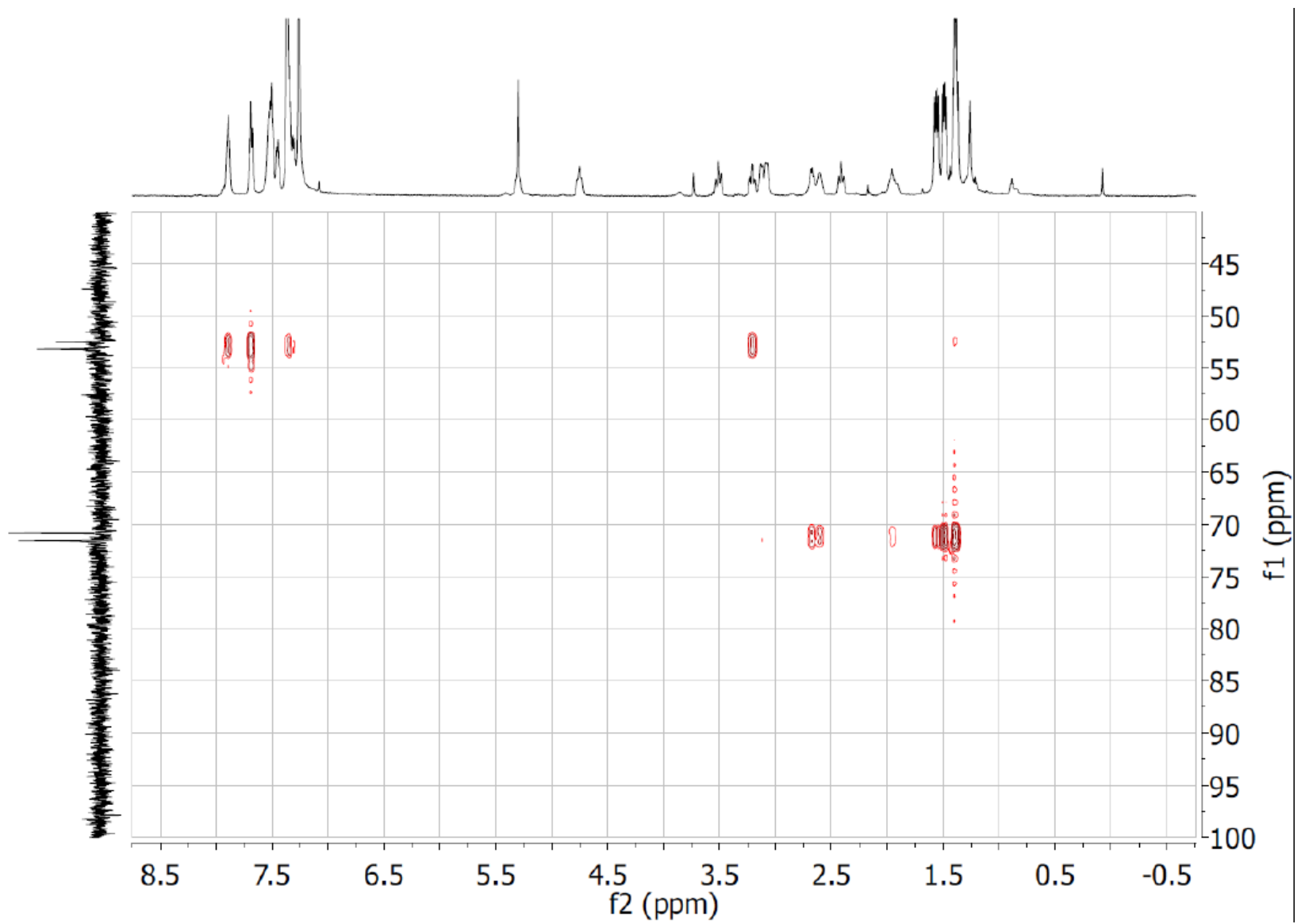

Figure S25: H-P HMBC (500 MHz, $\left.\mathrm{C}_{6} \mathrm{D}_{6}\right)$ of trans-Fe(Cl) $)_{2}(\mathrm{CO})\left[(\mathrm{S})-(\mathrm{iPr})_{2} \mathrm{PCH}_{2} \mathrm{CH}_{2} \mathrm{~N}(\mathrm{H}) \mathrm{CH}(\mathrm{Ph}) \mathrm{CH}_{2} \mathrm{P}(\mathrm{Ph})_{2}\right](\mathrm{S})-7$.

Synthesis of trans- $\mathrm{Fe}(\mathrm{Cl})_{2}(\mathrm{CO})[(\mathrm{S})-$

(iPr) $\left.{ }_{2} \mathrm{PCH}_{2} \mathrm{CH}_{2} \mathrm{~N}(\mathrm{H}) \mathrm{CH}\left(\mathrm{CH}\left(\mathrm{CH}_{3}\right)_{2}\right) \mathrm{CH}_{2} \mathrm{P}(\mathrm{Ph})_{2}\right](\mathrm{S})-8$ :

Follows the procedure above with (S)-(iPr) ${ }_{2} \mathrm{PCH}_{2} \mathrm{CH}_{2} \mathrm{~N}(\mathrm{H}) \mathrm{CH}(\mathrm{iPr}) \mathrm{CH}_{2} \mathrm{P}(\mathrm{Ph})_{2}(55 \mathrm{mg}, 0.132 \mathrm{mmol}), \mathrm{FeCl}_{2}(17$ $\mathrm{mg}, 0.132 \mathrm{mmol})$ and THF (10 mL). Yielded a purple solid (40 mg, 60\%). Crystals suitable for X-ray crystallography were obtained from a slow diffusion of pentane into benzene. ${ }^{1} \mathrm{H} N M R\left(\mathrm{C}_{6} \mathrm{D}_{6}, 500 \mathrm{MHz}\right)$ : $8.09(\mathrm{~m}, 2 \mathrm{H}, \mathrm{ArH}), 7.80(\mathrm{~m}, 2 \mathrm{H}, \mathrm{ArH}), 6.99(\mathrm{~m}, 6 \mathrm{H}, \mathrm{ArH}), 4.77(\mathrm{~m}, 1 \mathrm{H}, \mathrm{NH}), 3.83(\mathrm{~m}, 1 \mathrm{H}, \mathrm{CHiPr}), 3.00(\mathrm{~m}, 3 \mathrm{H}$, $\left.\mathrm{CH}_{2} \mathrm{CH}_{2} \mathrm{PiPr}_{2}, \mathrm{CH}_{2} \mathrm{PPh}_{2}\right), 2.77\left(\mathrm{~m}, 1 \mathrm{H}, \mathrm{PCH}\left(\mathrm{CH}_{3}\right)_{2}\right), 2.56\left(\mathrm{~m}, 2 \mathrm{H}, \mathrm{CH}_{2} \mathrm{PPh}_{2}, \mathrm{PCH}\left(\mathrm{CH}_{3}\right)_{2}\right), 2.21\left(\mathrm{~m}, 1 \mathrm{H}, \mathrm{CH}\left(\mathrm{CH}_{3}\right)_{2}\right)$, $2.02\left(\mathrm{~m}, 1 \mathrm{H}, \mathrm{CH}_{2} \mathrm{PiPr}_{2}\right), 1.64\left(\mathrm{~m}, 1 \mathrm{H}, \mathrm{CH}_{2} \mathrm{PiPr}_{2}\right), 1.57\left(\mathrm{~m}, 3 \mathrm{H}, \mathrm{PCH}\left(\mathrm{CH}_{3}\right)_{2}\right), 1.43\left(\mathrm{~m}, 3 \mathrm{H}, \mathrm{PCH}\left(\mathrm{CH}_{3}\right)_{2}\right), 1.37(\mathrm{~m}$, $\left.3 \mathrm{H}, \mathrm{PCH}\left(\mathrm{CH}_{3}\right)_{2}\right), 1.21\left(\mathrm{~m}, 3 \mathrm{H}, \mathrm{PCH}\left(\mathrm{CH}_{3}\right)_{2}\right), 0.89\left(\mathrm{~m}, 3 \mathrm{H}, \mathrm{CH}_{3}\right), 0.70\left(\mathrm{~m}, 3 \mathrm{H}, \mathrm{CH}_{3}\right) .{ }^{13} \mathrm{C}\left\{{ }^{1} \mathrm{H}\right\} \mathrm{NMR}\left(\mathrm{C}_{6} \mathrm{D}_{6}, 124\right.$ $\mathrm{MHz}): 224.7\left(\mathrm{t},{ }^{2} \mathrm{~J}_{\mathrm{CP}}=24 \mathrm{~Hz}, \mathrm{CO}\right), 134.2\left(\mathrm{~d}, J_{\mathrm{CP}}=9 \mathrm{~Hz}, C_{\mathrm{Ar}}\right), 132.3\left(\mathrm{~d}, J_{\mathrm{CP}}=8 \mathrm{~Hz}, C_{\mathrm{Ar}}\right), 129.9\left(C_{\mathrm{Ar}}\right), 129.1\left(C_{\mathrm{Ar}}\right)$, $128.4\left(C_{\mathrm{Ar}}\right), 63.3\left(\mathrm{~d}, J_{\mathrm{CP}}=8 \mathrm{~Hz}, \mathrm{CH}(\mathrm{iPr})\right), 45.3\left(\mathrm{CH}_{2} \mathrm{CH}_{2} \mathrm{PiPr}_{2}\right), 28.1\left(\mathrm{~d}, J_{\mathrm{CP}}=18 \mathrm{~Hz}, \mathrm{CH}_{2} \mathrm{PPh}_{2}\right), 27.3\left(\mathrm{CH}_{2} \mathrm{PiPr}_{2}\right)$, $27.2\left(\mathrm{CH}\left(\mathrm{CH}_{3}\right)_{2}\right), 23.9\left(\mathrm{~d}, J_{\mathrm{CP}}=22 \mathrm{~Hz}, \mathrm{PCH}\left(\mathrm{CH}_{3}\right)_{2}\right), 22.5\left(\mathrm{~d}, J_{\mathrm{CP}}=19 \mathrm{~Hz}, \mathrm{PCH}\left(\mathrm{CH}_{3}\right)_{2}\right), 21.6\left(\mathrm{CH}_{3}\left(\mathrm{CH}_{3}\right)_{2}\right), 20.4$ $\left(\mathrm{PCH}\left(\mathrm{CH}_{3}\right)_{2}\right), 20.1\left(\mathrm{PCH}\left(\mathrm{CH}_{3}\right)_{2}\right), 19.4\left(\mathrm{PCH}\left(\mathrm{CH}_{3}\right)_{2}\right), 18.8\left(\mathrm{PCH}\left(\mathrm{CH}_{3}\right)_{2}\right), 13.5\left(\mathrm{CH}\left(\mathrm{CH}_{3}\right)_{2}\right) .{ }^{1} \mathrm{P}\left\{{ }^{1} \mathrm{H}\right\} \mathrm{NMR}\left(\mathrm{C}_{6} \mathrm{D}_{6}, 202\right.$ $\mathrm{MHz}): 69.3\left(\mathrm{~d},{ }^{2} J_{\mathrm{PP}}=177 \mathrm{~Hz}, \mathrm{PiPr}_{2}\right), 53.3\left(\mathrm{~d},{ }^{2} J_{\mathrm{PP}}=177 \mathrm{~Hz}, P \mathrm{Ph}_{2}\right) ;$ AT-IR $1933 \mathrm{~cm}^{-1} v_{(\mathrm{CO})}$. 


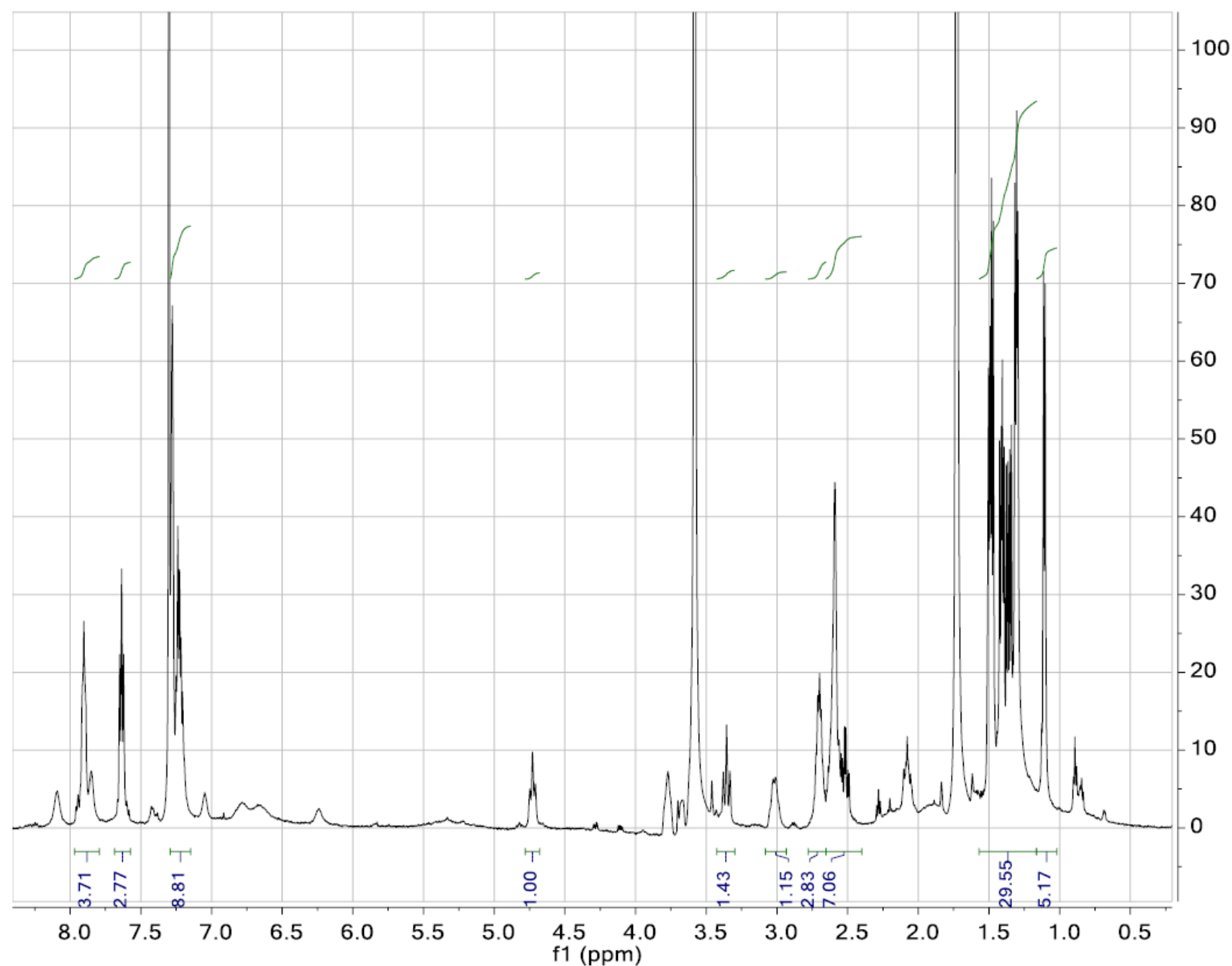

Figure $\quad \mathrm{S} 26: \quad{ }^{1} \mathrm{H} \quad \mathrm{NMR} \quad$ spectrum $\quad\left(500 \quad \mathrm{MHz}, \quad \mathrm{C}_{6} \mathrm{D}_{6}\right) \quad$ of trans-Fe $(\mathrm{Cl})_{2}(\mathrm{CO})[(\mathrm{S}, \mathrm{S})$ (iPr) $\left.{ }_{2} \mathrm{PCH}_{2} \mathrm{CH}_{2} \mathrm{~N}(\mathrm{H}) \mathrm{CH}\left(\mathrm{CH}\left(\mathrm{CH}_{3}\right)_{2}\right) \mathrm{CH}_{2} \mathrm{P}(\mathrm{Ph})_{2}\right](S)-8$. 


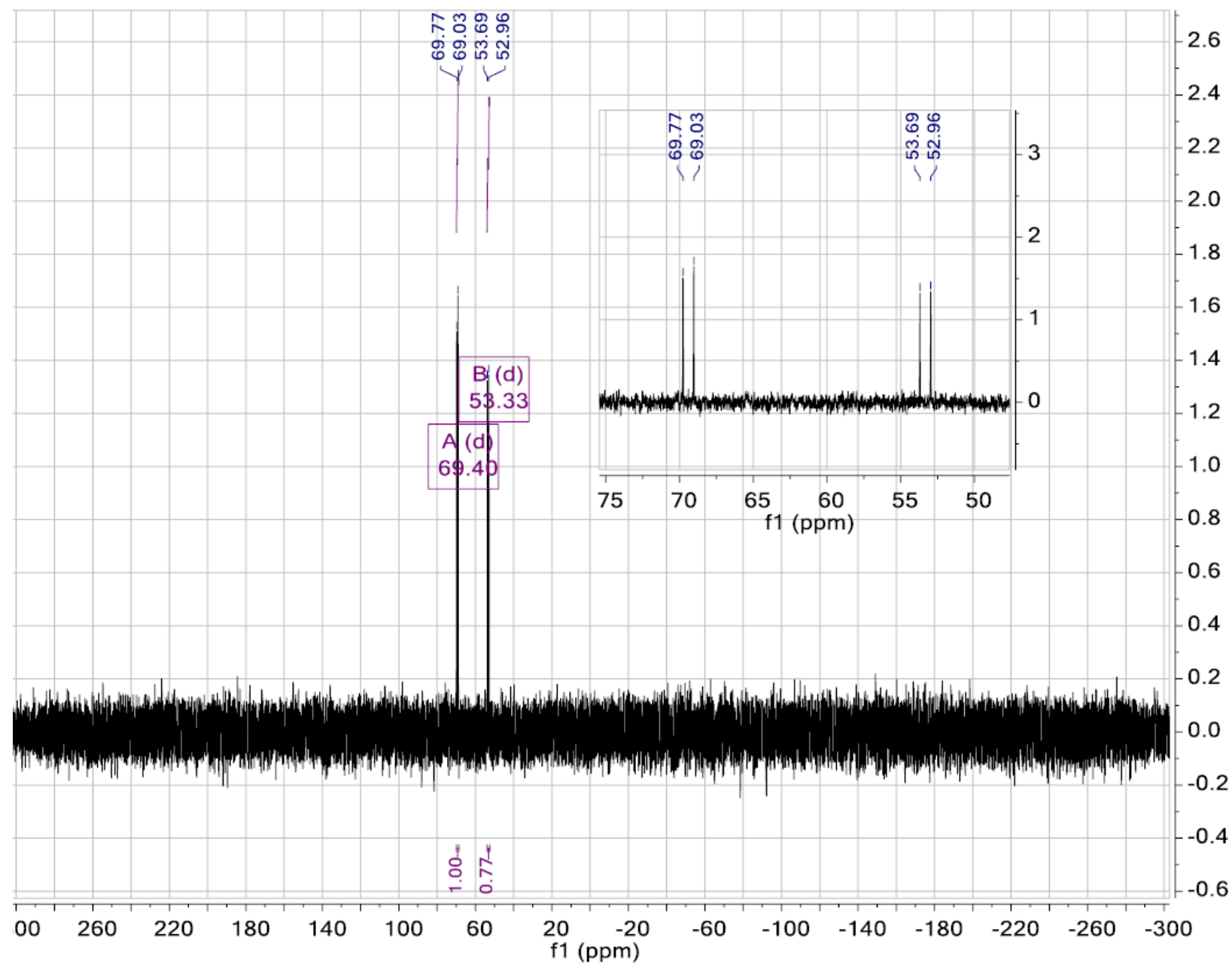

Figure S27: ${ }^{31} \mathrm{P}\left\{{ }^{1} \mathrm{H}\right\} \quad \mathrm{NMR}$ spectrum $\left(202 \quad \mathrm{MHz}, \quad \mathrm{C}_{6} \mathrm{D}_{6}\right)$ of trans- $\mathrm{Fe}(\mathrm{Cl})_{2}(\mathrm{CO})[(\mathrm{S}, \mathrm{S})$ (iPr) $\left.{ }_{2} \mathrm{PCH}_{2} \mathrm{CH}_{2} \mathrm{~N}(\mathrm{H}) \mathrm{CH}\left(\mathrm{CH}\left(\mathrm{CH}_{3}\right)_{2}\right) \mathrm{CH}_{2} \mathrm{P}(\mathrm{Ph})_{2}\right](\mathrm{S})-8$. 


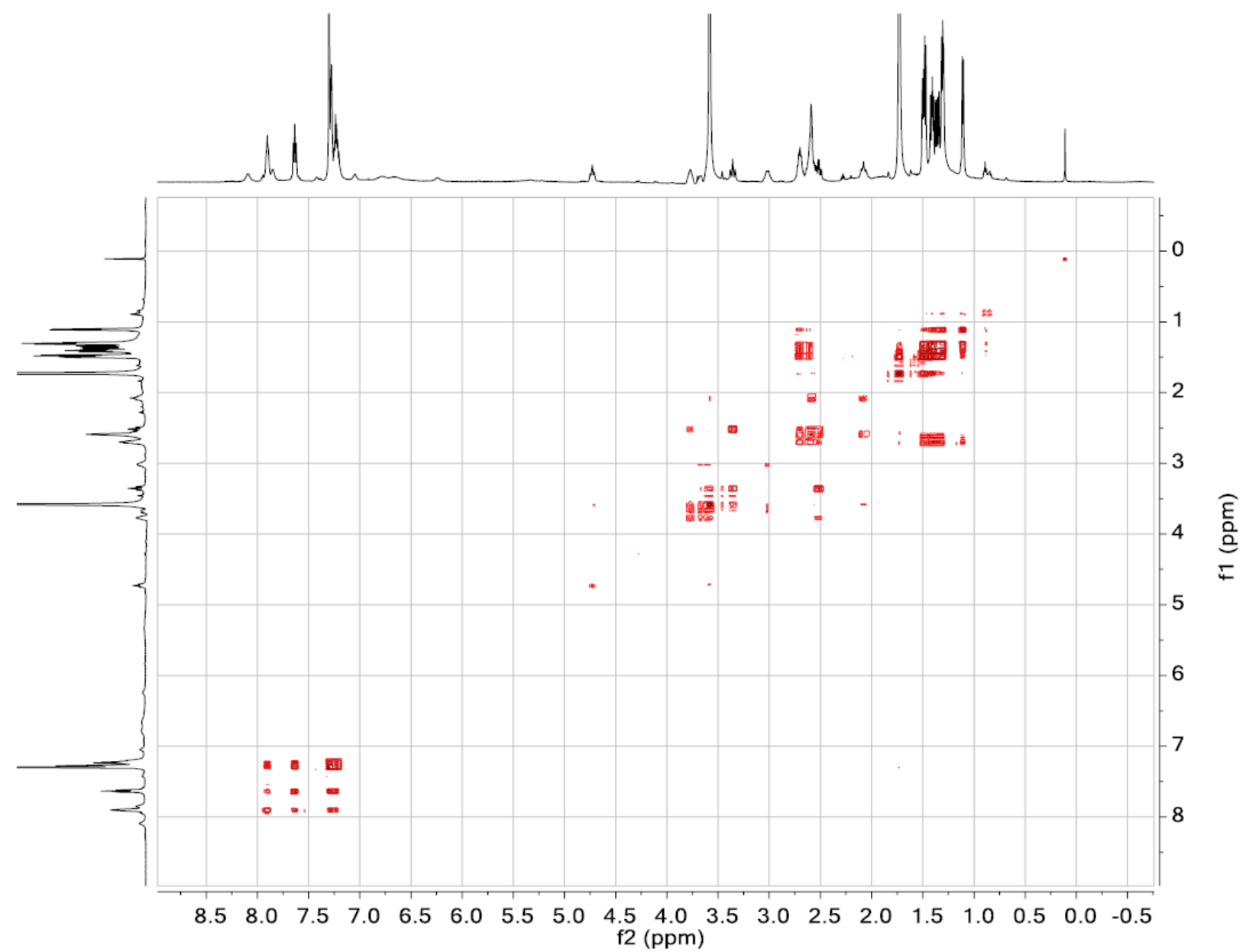

Figure $\mathrm{S} 28: \quad{ }^{1} \mathrm{H} \quad \mathrm{COSY} \quad \mathrm{NMR}$ spectrum $\left(500 \quad \mathrm{MHz}, \quad \mathrm{C}_{6} \mathrm{D}_{6}\right)$ of trans- $\mathrm{Fe}(\mathrm{Cl})_{2}(\mathrm{CO})[(\mathrm{S}, \mathrm{S})$ (iPr) $\left.{ }_{2} \mathrm{PCH}_{2} \mathrm{CH}_{2} \mathrm{~N}(\mathrm{H}) \mathrm{CH}\left(\mathrm{CH}\left(\mathrm{CH}_{3}\right)_{2}\right) \mathrm{CH}_{2} \mathrm{P}(\mathrm{Ph})_{2}\right](\mathrm{S})-8$.

\section{Synthesis of $\mathrm{Fe}(\mathrm{H})(\mathrm{Cl})(\mathrm{CO})\left[(\mathrm{S}, \mathrm{S})-(\mathrm{iPr})_{2} \mathrm{PCH}_{2} \mathrm{CH}_{2} \mathrm{~N}(\mathrm{H}) \mathrm{CH}(\mathrm{Ph}) \mathrm{CH}(\mathrm{Ph}) \mathrm{P}(\mathrm{Ph})_{2}\right]$} (S,S)-9:

A vial was charged with $(S, S)-5(83 \mathrm{mg}, 0.122 \mathrm{mmol})$, THF $(10 \mathrm{~mL})$ placed in a freezer $\left(-30^{\circ} \mathrm{C}\right)$ for $20 \mathrm{~min}$. The vial was removed and $\mathrm{LiHBEt}_{3}(135 \mu \mathrm{L}, 1 \mathrm{M}$ THF solution, $0.134 \mathrm{mmol})$ was added. The colour of the solution immediately changed from purple to red-orange. The reaction was allowed to stir to room temperature. The solvent was removed, taken up with benzene and subsequently filtered into a preweighed vial. Benzene was removed by lyophilization to yield an orange-brown solid $(70 \mathrm{mg}, 89 \%) .{ }^{1} \mathrm{H}$ NMR (THF-d $\left.d_{8}, 500 \mathrm{MHz}\right):-20.46\left(\mathrm{dd},{ }^{2} J_{\mathrm{HP}}=47,56 \mathrm{~Hz}, \mathrm{FeH}\right.$, minor hydride $\left.9 \%\right),-21.48\left(\mathrm{dd},{ }^{2} J_{\mathrm{HP}}=47,56 \mathrm{~Hz}\right.$, FeH, major hydride 87\%), -22.12 (dd, ${ }^{2} J_{\mathrm{HP}}=57,60 \mathrm{~Hz}, \mathrm{FeH}$, minor hydride $4 \%$ ). ${ }^{31} \mathrm{P}\left\{{ }^{1} \mathrm{H}\right\} \mathrm{NMR}\left(\mathrm{THF}-\mathrm{d}_{8}, 202\right.$ $\mathrm{MHz}): 94.5\left(\mathrm{dd},{ }^{2} J_{\mathrm{PP}}=138 \mathrm{~Hz},{ }^{2} J_{\mathrm{HP}}\right.$ residual $\left.=33 \mathrm{~Hz}, P \mathrm{PPr} 2\right), 92.2\left(\mathrm{dd},{ }^{2} J_{\mathrm{PP}}=138 \mathrm{~Hz},{ }^{2} J_{\mathrm{HP}}\right.$ residual $\left.=26 \mathrm{~Hz}, P \mathrm{Ph}_{2}\right)$. ATIR $2115 \mathrm{~cm}^{-1} v_{(\mathrm{Fe}-\mathrm{H}),} 1900 \mathrm{~cm}^{-1} v_{(\mathrm{CO})}$. EA Calc. for $\mathrm{C}_{35} \mathrm{H}_{42} \mathrm{ClFeNOP}_{2} . \mathrm{C}_{6} \mathrm{H}_{6}: \mathrm{C}, 68.0 ; \mathrm{H}, 6.7 ; \mathrm{N}, 1.9$. Found: C, 67.6; $\mathrm{H}, 6.9 ; \mathrm{N}, 2.3$. The hydride regions of the ${ }^{1} \mathrm{H}$ NMR spectra were simulated to obtain accurate populations of the 3 isomers. 

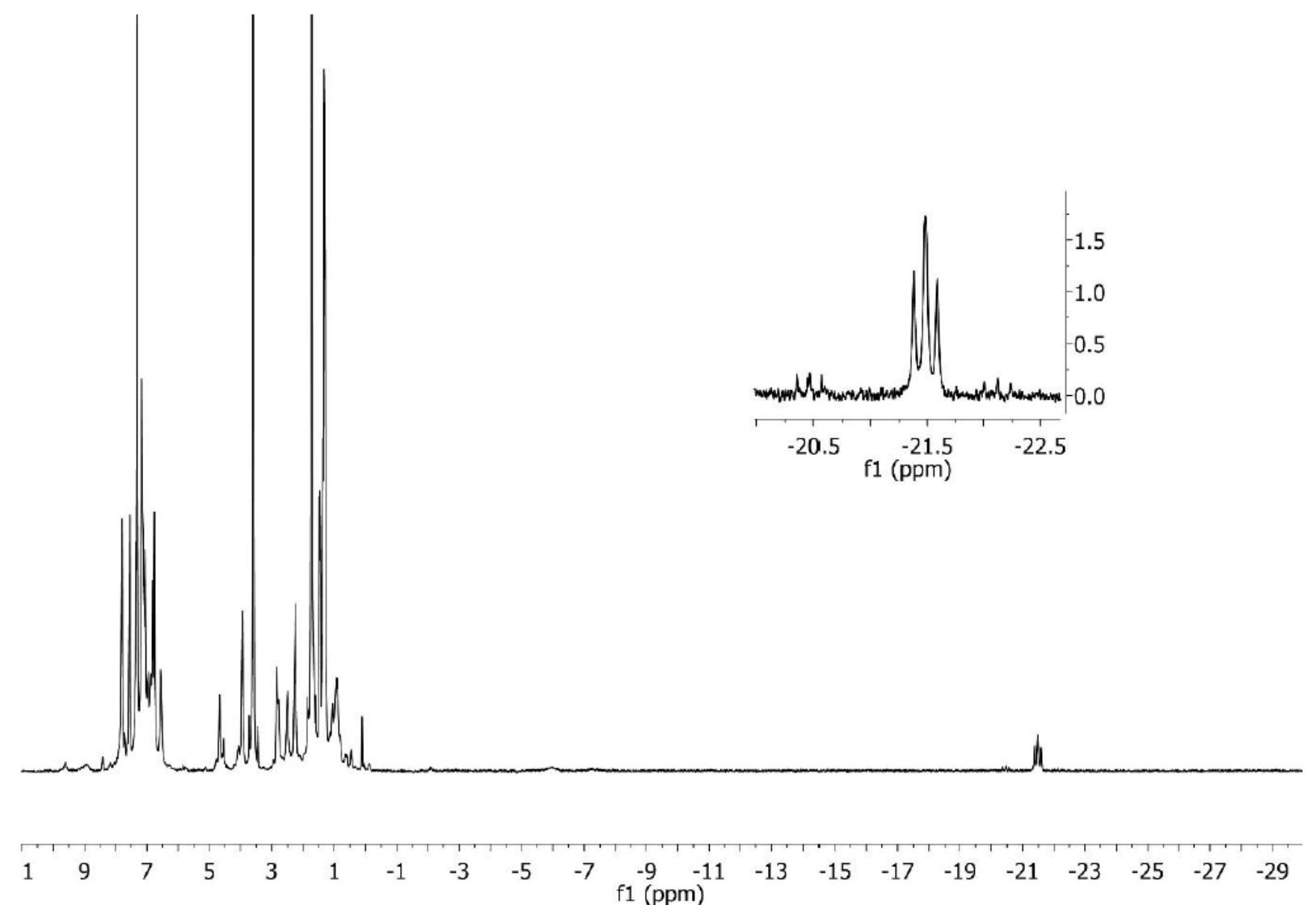

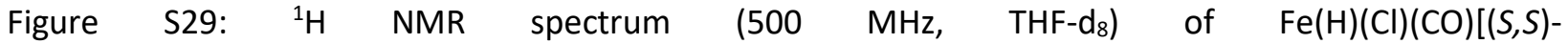
(iPr) $\left.{ }_{2} \mathrm{PCH}_{2} \mathrm{CH}_{2} \mathrm{~N}(\mathrm{H}) \mathrm{CH}(\mathrm{Ph}) \mathrm{CH}(\mathrm{Ph}) \mathrm{P}(\mathrm{Ph})_{2}\right](\boldsymbol{S}, \boldsymbol{S})$-9. 


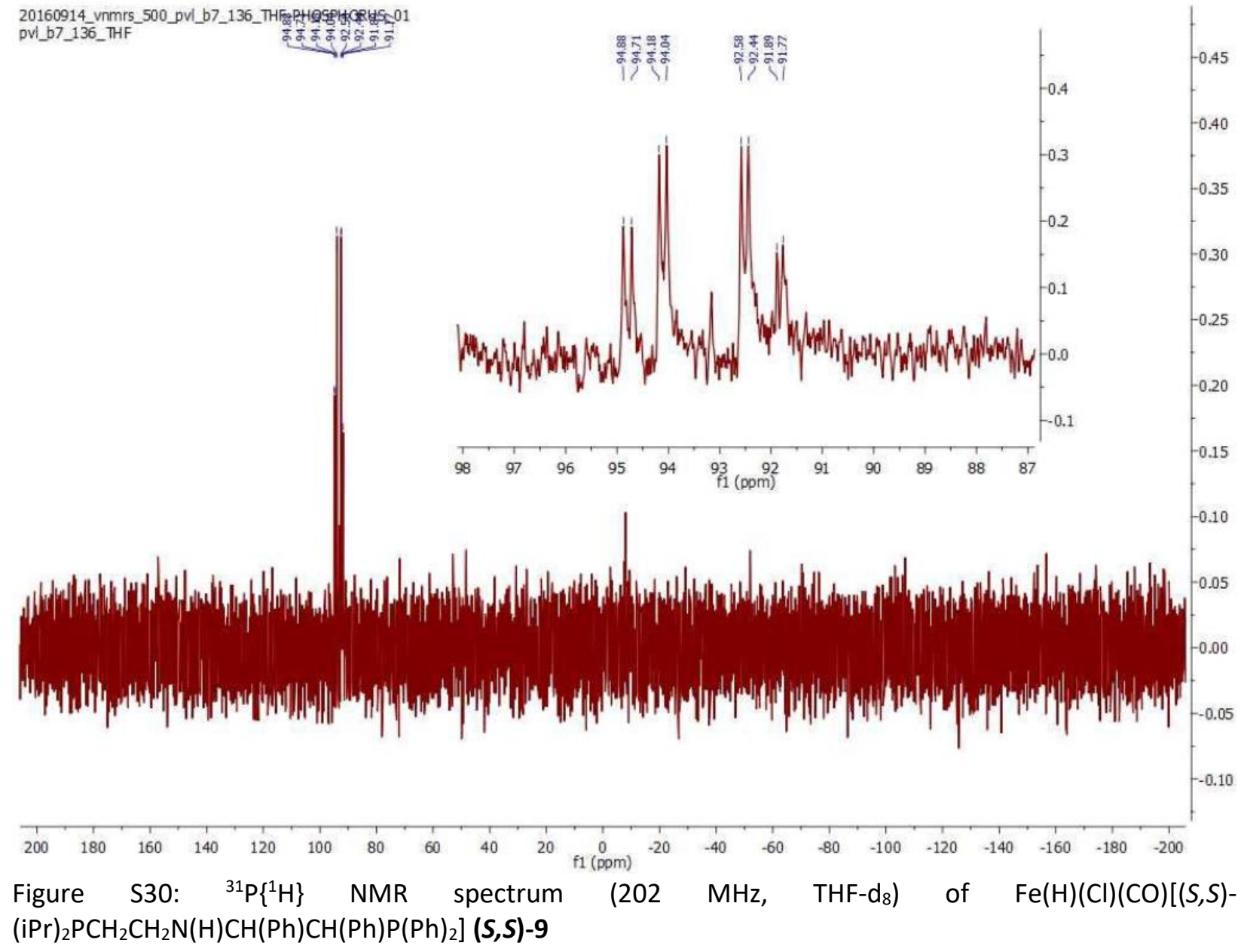




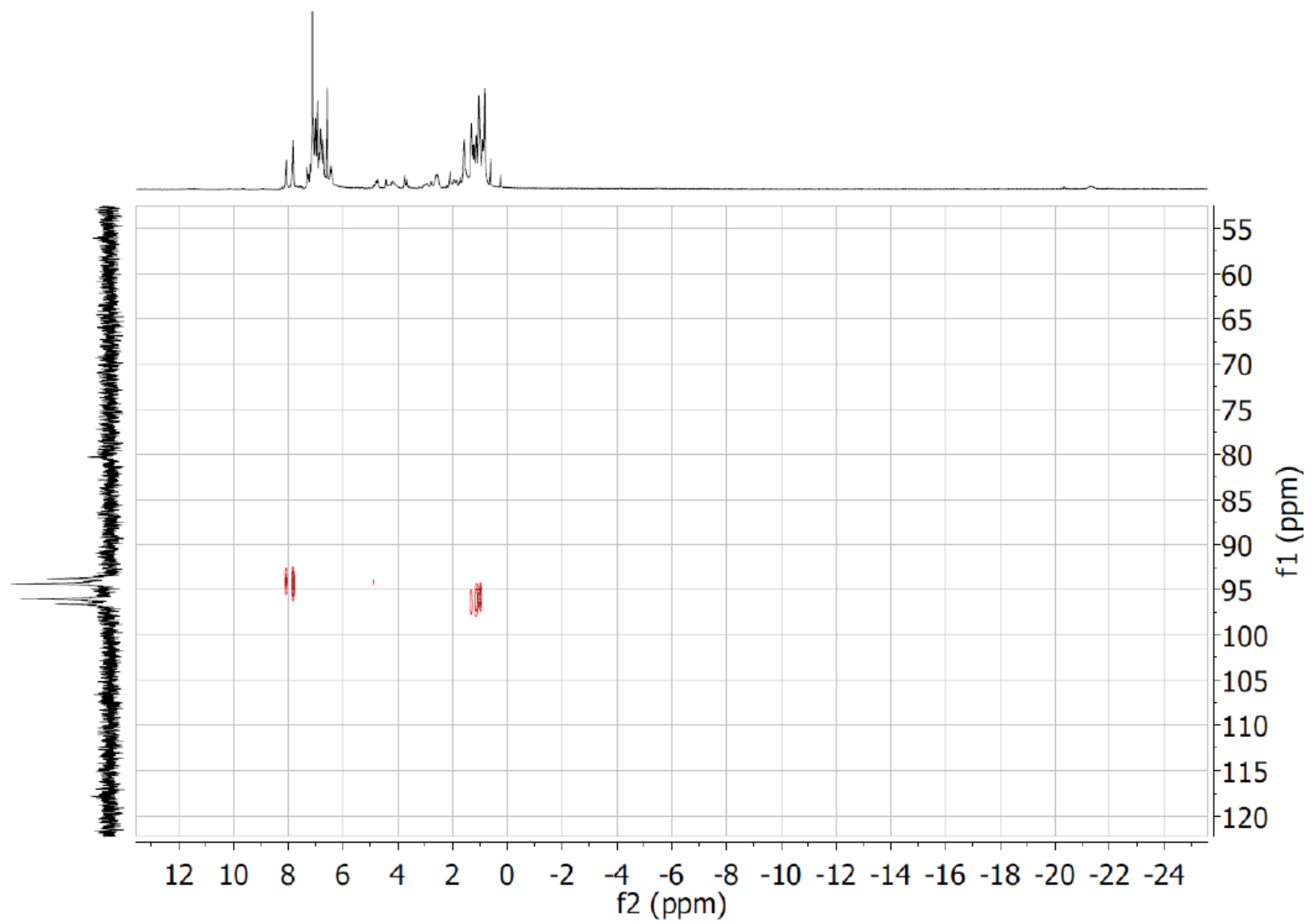

Figure S31: H-P HMBC (500 MHz, THF-d $\left.\mathrm{d}_{8}\right)$ of $\mathrm{Fe}(\mathrm{H})(\mathrm{Cl})(\mathrm{CO})\left[(\mathrm{S}, \mathrm{S})-(\mathrm{PPr})_{2} \mathrm{PCH}_{2} \mathrm{CH}_{2} \mathrm{~N}(\mathrm{H}) \mathrm{CH}(\mathrm{Ph}) \mathrm{CH}(\mathrm{Ph}) \mathrm{P}(\mathrm{Ph})_{2}\right]$ $(S, S)-9$.

Synthesis of $\mathrm{Fe}(\mathrm{H})(\mathrm{Cl})(\mathrm{CO})\left[(\mathrm{S}, \mathrm{S})-(\mathrm{Cy})_{2} \mathrm{PCH}_{2} \mathrm{CH}_{2} \mathrm{~N}(\mathrm{H}) \mathrm{CH}(\mathrm{Ph}) \mathrm{CH}(\mathrm{Ph}) \mathrm{P}(\mathrm{Ph})_{2}\right]$ :

Follows the procedure above with $(S, S)-6\left(44 \mathrm{mg}, 5.77 \times 10^{-2} \mathrm{mmol}\right.$ ) and LiHBEt $_{3}(64 \mu \mathrm{L}, 1 \mathrm{M}$ THF solution, $\left.6.35 \times 10^{-2} \mathrm{mmol}\right)$. Yielded a red solid (42 mg, quant.). ${ }^{1} \mathrm{H}$ NMR $\left(\mathrm{C}_{6} \mathrm{D}_{6}, 500 \mathrm{MHz}\right):-21.57\left(\mathrm{dd},{ }^{2} J_{\mathrm{HP}}=48,56\right.$ $\mathrm{Hz}, \mathrm{FeH}) .{ }^{31} \mathrm{P}\left\{{ }^{1} \mathrm{H}\right\} \mathrm{NMR}\left(\mathrm{C}_{6} \mathrm{D}_{6}, 202 \mathrm{MHz}\right): 94.3\left(\mathrm{dd},{ }^{2} J_{\mathrm{PP}}=137 \mathrm{~Hz},{ }^{2} J_{\mathrm{HP}}{ }^{\text {residual }}=18 \mathrm{~Hz}, \mathrm{PiPr}_{2}\right), 87.8\left(\mathrm{dd},{ }^{2} J_{\mathrm{PP}}=141\right.$ $\mathrm{Hz},{ }^{2} J_{\mathrm{HP}}$ residual $=22 \mathrm{~Hz}, P P \mathrm{Ph}_{2}$ ). AT-IR $1898 \mathrm{~cm}^{-1} v_{(\mathrm{CO})}$. EA Calc. for $\mathrm{C}_{41} \mathrm{H}_{50} \mathrm{ClFeNOP}$. $2.0 \mathrm{C}_{6} \mathrm{H}_{6}: \mathrm{C}, 72.14 ; \mathrm{H}, 7.10$; N, 1.59. Found: C, 72.32; $H, 7.40 ; N, 1.81$. 


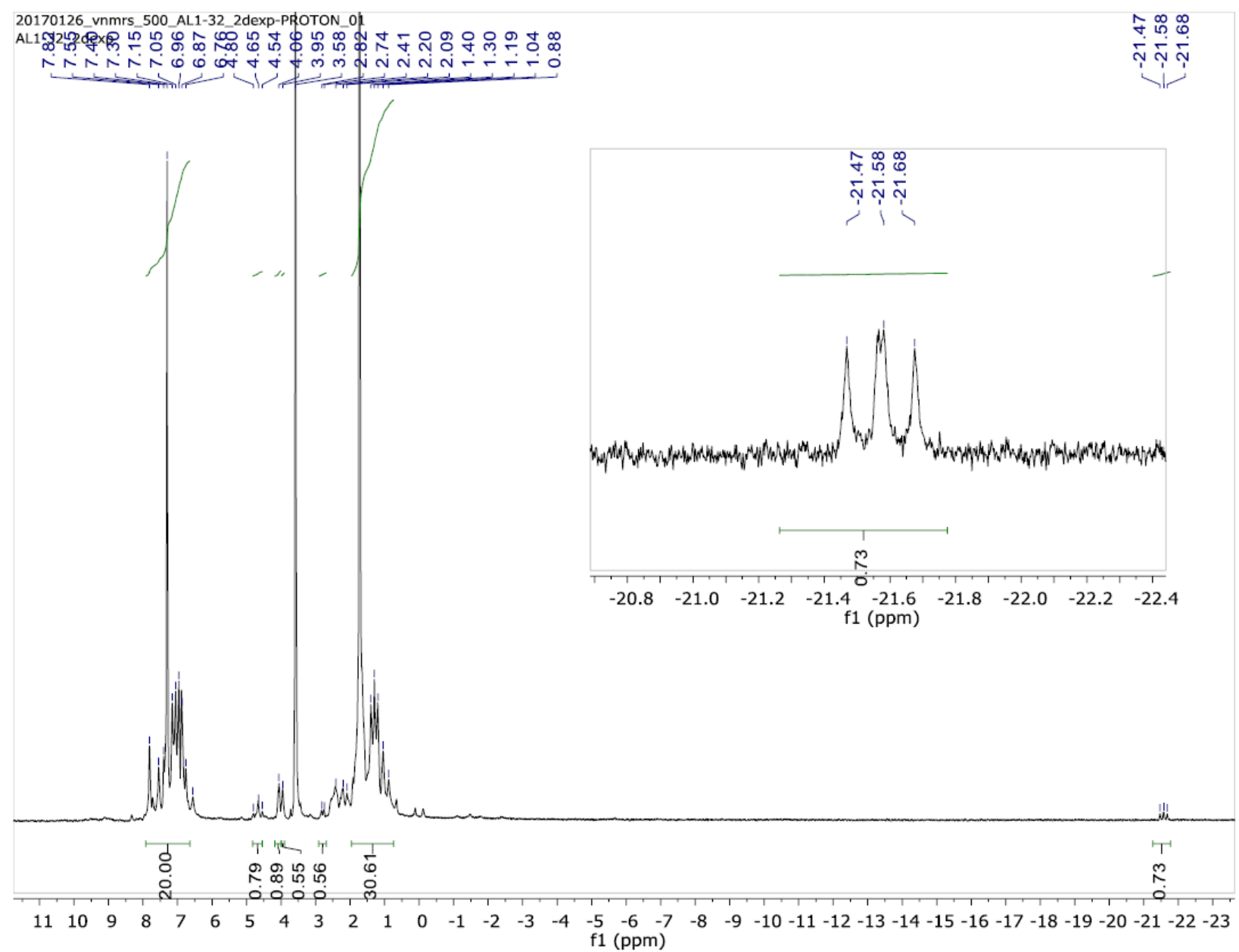

Figure $\quad \mathrm{S} 32: \quad{ }^{1} \mathrm{H} \quad \mathrm{NMR} \quad$ spectrum $\left(500 \quad \mathrm{MHz}, \quad \mathrm{C}_{6} \mathrm{D}_{6}\right)$ of trans- $\mathrm{Fe}(\mathrm{H})(\mathrm{Cl})(\mathrm{CO})[(\mathrm{S}, \mathrm{S})$ $\left.(\mathrm{Cy})_{2} \mathrm{PCH}_{2} \mathrm{CH}_{2} \mathrm{~N}(\mathrm{H}) \mathrm{CH}(\mathrm{Ph}) \mathrm{CH}(\mathrm{Ph}) \mathrm{P}(\mathrm{Ph})_{2}\right]$. 


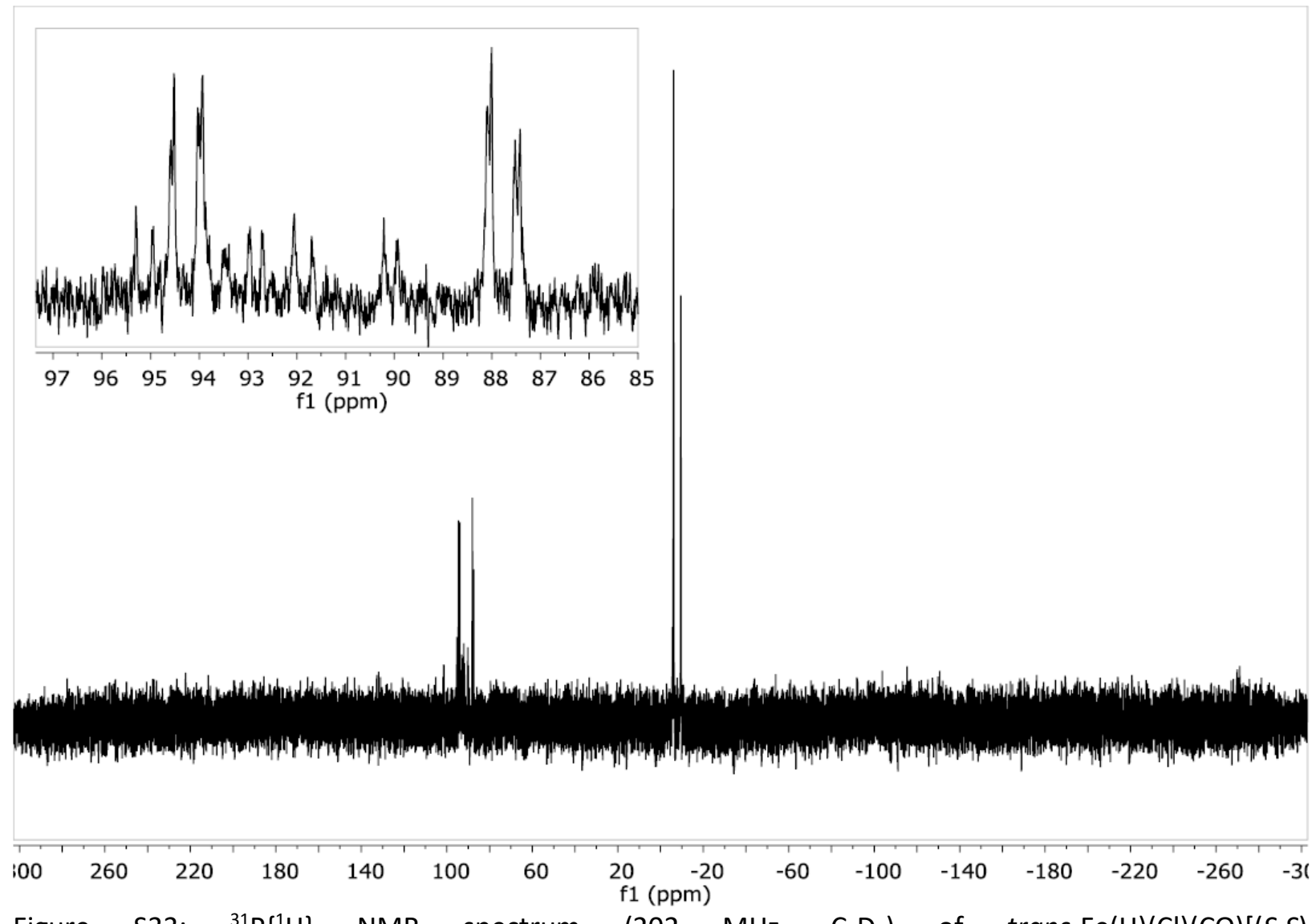

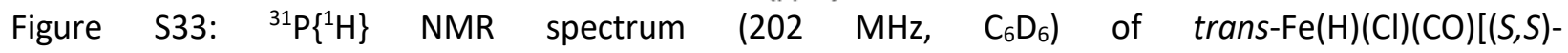
(Cy) $\left.{ }_{2} \mathrm{PCH}_{2} \mathrm{CH}_{2} \mathrm{~N}(\mathrm{H}) \mathrm{CH}(\mathrm{Ph}) \mathrm{CH}(\mathrm{Ph}) \mathrm{P}(\mathrm{Ph})_{2}\right]$.

\section{Synthesis of $\mathrm{Fe}(\mathrm{H})(\mathrm{Cl})(\mathrm{CO})\left[(\mathrm{S})-(\mathrm{iPr})_{2} \mathrm{PCH}_{2} \mathrm{CH}_{2} \mathrm{~N}(\mathrm{H}) \mathrm{CH}(\mathrm{Ph}) \mathrm{CH}_{2} \mathrm{P}(\mathrm{Ph})_{2}\right](\mathrm{S})-10$ :}

A vial was charged with $(S)-7(40 \mathrm{mg}, 0.066 \mathrm{mmol})$, THF $(10 \mathrm{~mL})$ and placed in a freezer $\left(-30^{\circ} \mathrm{C}\right)$ for $20 \mathrm{~min}$. The vial was removed and LiHBEt $3(73 \mu \mathrm{L}, 1 \mathrm{M}$ THF solution, $0.073 \mathrm{mmol}$ ) was added. The colour of the solution immediately changed from purple to red-orange. The reaction was allowed to stir to room temperature. The solvent was removed, taken up with benzene and subsequently filtered into a preweighed vial. Benzene was removed by lyophilization to yield an orange-brown solid (40 mg, quant.). Crystals suitable for X-ray diffraction were obtained from slow diffusion of pentane into benzene. Four isomers were detected by NMR spectroscopy as determined by four separate hydride signals. ${ }^{1} \mathrm{H}$ NMR $\left(\mathrm{C}_{6} \mathrm{D}_{6}, 500 \mathrm{MHz}\right):-20.47\left(\mathrm{t},{ }^{2} J_{\mathrm{HP}}=55 \mathrm{~Hz}, \mathrm{FeH}, 5 \%\right),-20.96\left(\mathrm{dd},{ }^{2} J_{\mathrm{HP}}=52,56 \mathrm{~Hz}, \mathrm{FeH}, 22 \%\right),-21.40\left(\mathrm{t},{ }^{2} J_{\mathrm{HP}}=\right.$ $54 \mathrm{~Hz}, \mathrm{FeH}, 31 \%),-22.05$ (dd, $\left.{ }^{2} J_{\mathrm{HP}}=53,55 \mathrm{~Hz}, \mathrm{FeH}, 42 \%\right) .{ }^{31} \mathrm{P}\left\{{ }^{1} \mathrm{H}\right\} \mathrm{NMR}\left(\mathrm{C}_{6} \mathrm{D}_{6}, 202 \mathrm{MHz}\right.$, overlapping multiplets): $65-66$ (m, $P \mathrm{Ph}_{2}, 3$ isomers), 68.9 (dd, ${ }^{2} J_{\mathrm{PP}}=136,{ }^{2} J_{\mathrm{HP}}$ residual $=33 \mathrm{~Hz}, P \mathrm{Ph}_{2}, 1$ isomer), 93-95 ppm (m, $\mathrm{PiPr}_{2}, 4$ isomers). AT-IR $1897 \mathrm{~cm}^{-1} v_{(\mathrm{CO})}$. EA Found Calcd. for $\mathrm{C}_{29} \mathrm{H}_{38} \mathrm{ClFeNOP}_{2} 0.5 \mathrm{C}_{6} \mathrm{H}_{6}: \mathrm{C}, 63.1 ; \mathrm{H}, 6.8 ; \mathrm{N}$, 2.3. Found $\mathrm{C}, 62.7 ; \mathrm{H}, 6.9 ; \mathrm{N}, 2.2$. The hydride regions of the ${ }^{1} \mathrm{H}$ NMR spectra were simulated to obtain accurate populations of the 4 isomers. 


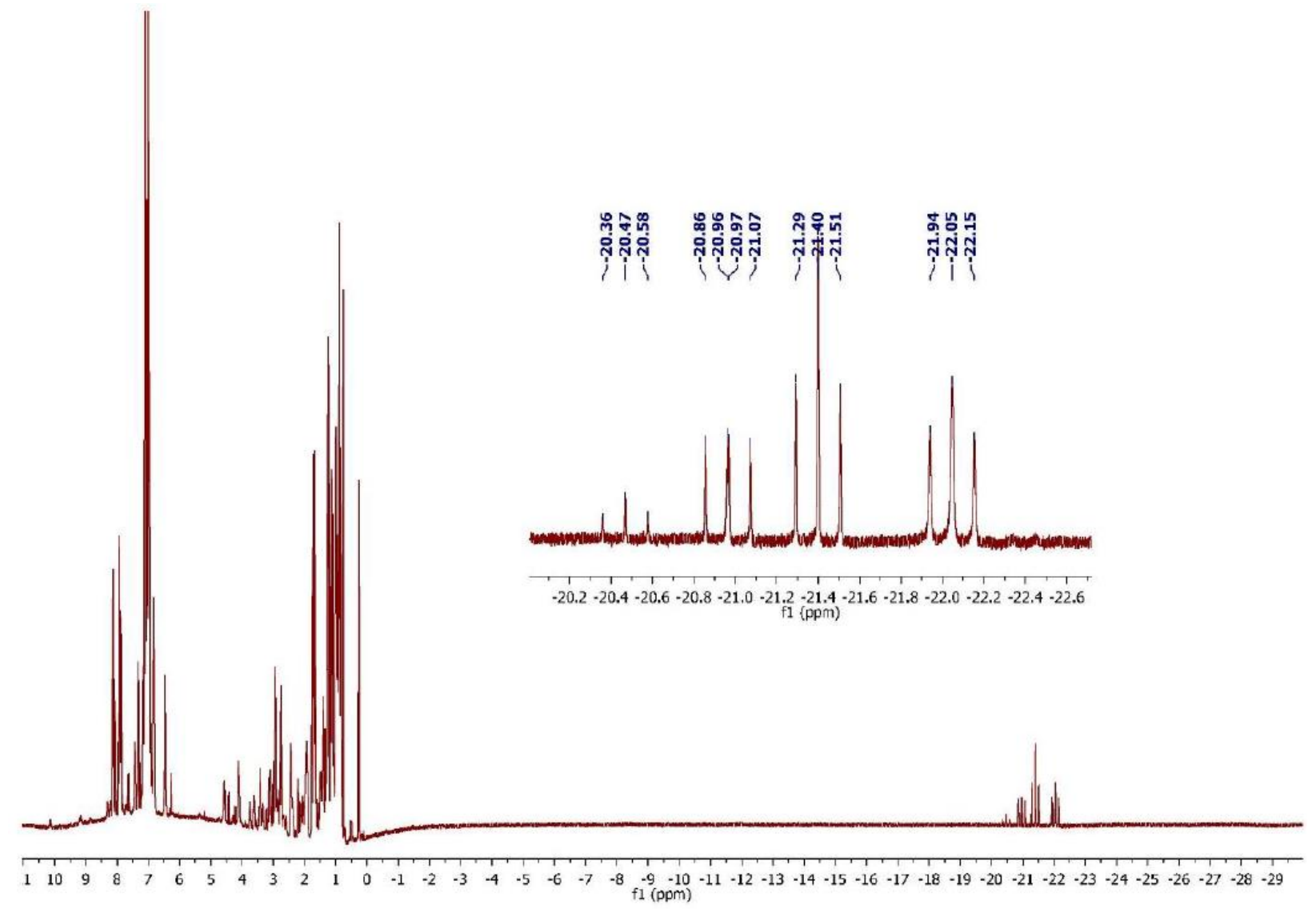

Figure S34: ${ }^{1} \mathrm{H} \quad \mathrm{NMR}$ spectrum $\left(500 \mathrm{MHz}, \mathrm{C}_{6} \mathrm{D}_{6}\right)$, hydride region of $\mathrm{Fe}(\mathrm{H})(\mathrm{Cl})(\mathrm{CO})[(\mathrm{S})$ (iPr) $\left.{ }_{2} \mathrm{PCH}_{2} \mathrm{CH}_{2} \mathrm{~N}(\mathrm{H}) \mathrm{CH}(\mathrm{Ph}) \mathrm{CH}_{2} \mathrm{P}(\mathrm{Ph})_{2}\right](\mathrm{S})-\mathbf{1 0}$. 


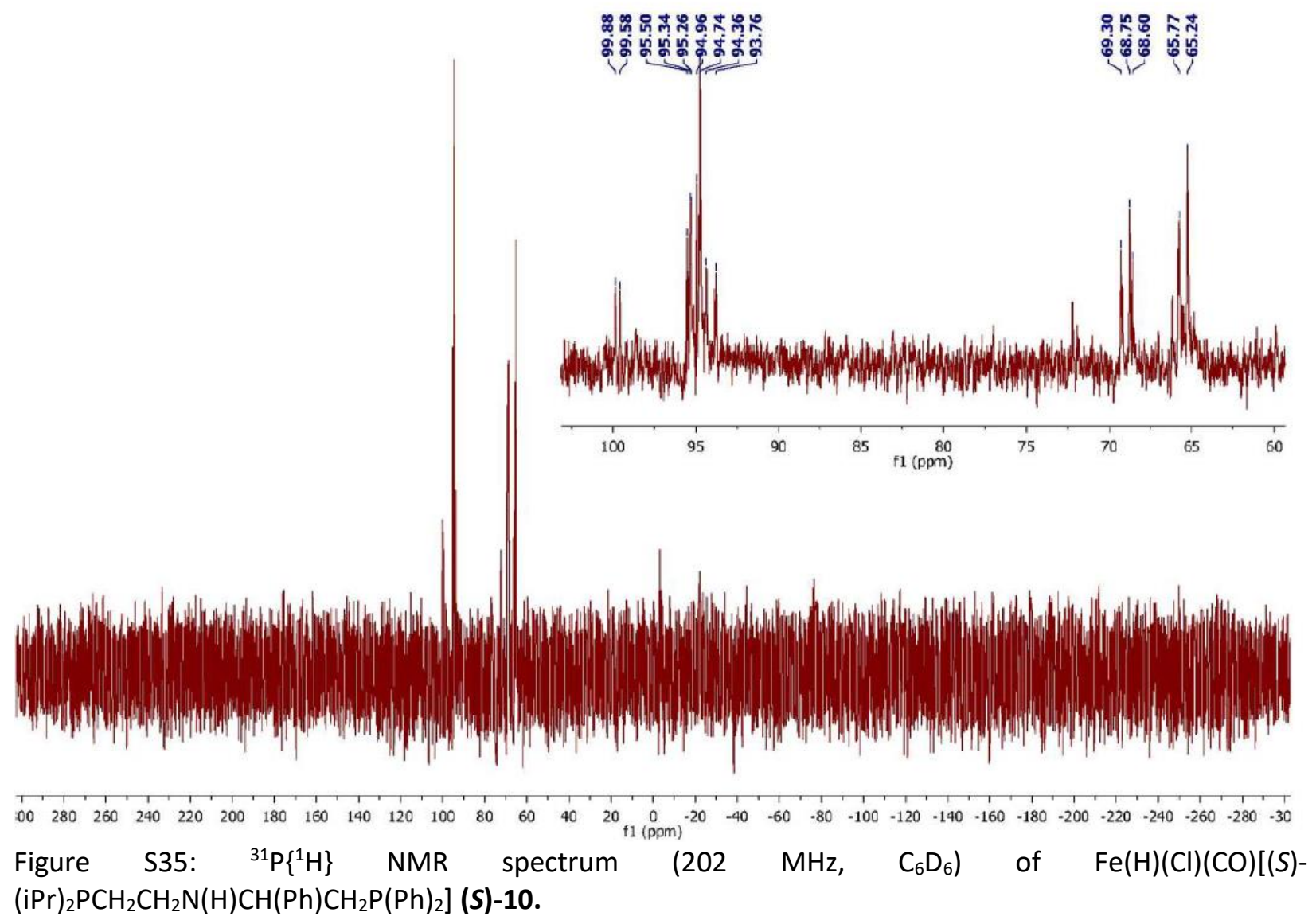

Synthesis of $\mathrm{Fe}(\mathrm{H})(\mathrm{Cl})(\mathrm{CO})\left[(\mathrm{S})-(\mathrm{iPr})_{2} \mathrm{PCH}_{2} \mathrm{CH}_{2} \mathrm{~N}(\mathrm{H}) \mathrm{CH}(\mathrm{iPr}) \mathrm{CH}_{2} \mathrm{P}(\mathrm{Ph})_{2}\right](\mathrm{S})-11$ : Follows the procedure above with $(S)-8(75 \mathrm{mg}, 0.131 \mathrm{mmol})$ and $\mathrm{LiHBEt}_{3}(131 \mu \mathrm{L}, 1 \mathrm{M}$ THF solution, 0.131 $\mathrm{mmol})$. Yielded an orange-brown solid (70 $\mathrm{mg}$, quant.). Crystals suitable for X-ray diffraction were obtained from slow diffusion of pentane into benzene. At least 6 isomers were detected by NMR spectroscopy as determined by seven separate hydride signals, two of which are correlated in ${ }^{1} \mathrm{H}-{ }^{1} \mathrm{H}$ COSY NMR. ${ }^{1} \mathrm{H}$ NMR $\left(\mathrm{C}_{6} \mathrm{D}_{6}, 500 \mathrm{MHz}\right):-8.11(\mathrm{~m}, \mathrm{FeH}, 7 \%),-8.41$ (m, FeH, 14\%), -13.41 (bm, FeH, 11\%), -14.51 (bm, FeH, 9\%), -20.96 (dd, $\left.{ }^{2} J_{H P}=56.9,52.5 \mathrm{~Hz}, \mathrm{FeH}, 17 \%\right),-22.04$ (dd, ${ }^{2} J_{\mathrm{HP}}=55.8,53 \mathrm{~Hz}, \mathrm{FeH}, 21 \%$ ), -22.78 $\left(\mathrm{t},{ }^{2} J_{\mathrm{HP}}=60.3 \mathrm{~Hz}, \mathrm{FeH}, 17 \%\right) .{ }^{31} \mathrm{P}\left\{{ }^{1} \mathrm{H}\right\} \mathrm{NMR}\left(\mathrm{C}_{6} \mathrm{D}_{6}, 202 \mathrm{MHz}\right.$, extracted from HMBC): 64.68 (d, $P \mathrm{Ph}_{2}$, correlated to $\mathrm{FeH}$ at -20.96), 64.62 (d, $P \mathrm{Ph}_{2}$, correlated to $\mathrm{FeH}$ at -22.05), 91.20 (d, $P \mathrm{PPr}_{2}$, correlated to $\mathrm{FeH}$ at -20.96), 92.08 (d, $\mathrm{PiPr}_{2}$, correlated to $\mathrm{FeH}$ at -22.03 ). 


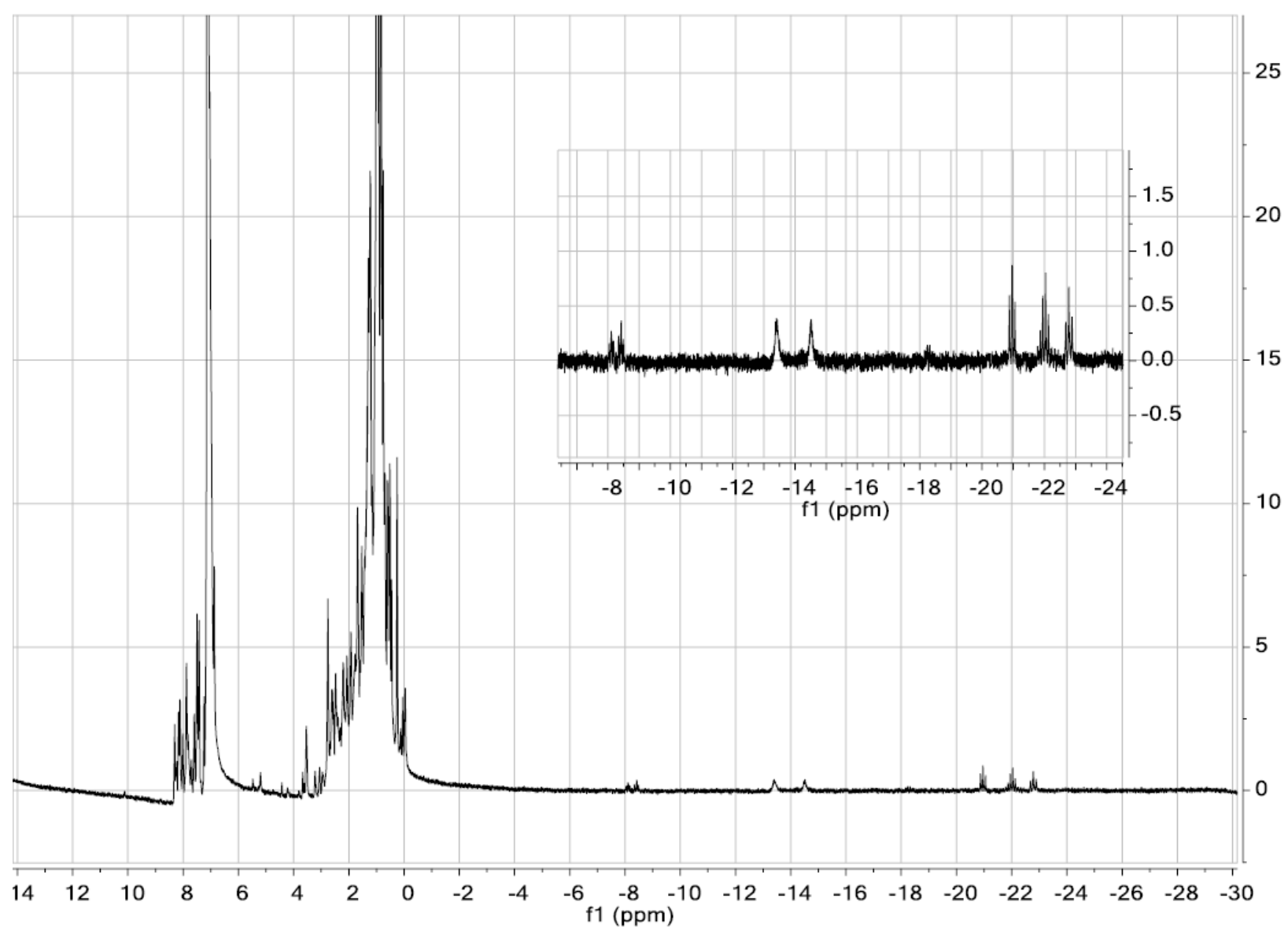

Figure $\quad \mathrm{S} 36:{ }^{1} \mathrm{H} \quad \mathrm{NMR}$ spectrum $\left(500 \quad \mathrm{MHz}, \quad \mathrm{C}_{6} \mathrm{D}_{6}\right)$ of trans- $\mathrm{Fe}(\mathrm{H})(\mathrm{Cl})(\mathrm{CO})[(\mathrm{S})$ (iPr) $\left.{ }_{2} \mathrm{PCH}_{2} \mathrm{CH}_{2} \mathrm{~N}(\mathrm{H}) \mathrm{CH}\left(\mathrm{CH}\left(\mathrm{CH}_{3}\right)_{2}\right) \mathrm{CH}_{2} \mathrm{P}(\mathrm{Ph})_{2}\right](\mathrm{S})-\mathbf{1 1}$. 


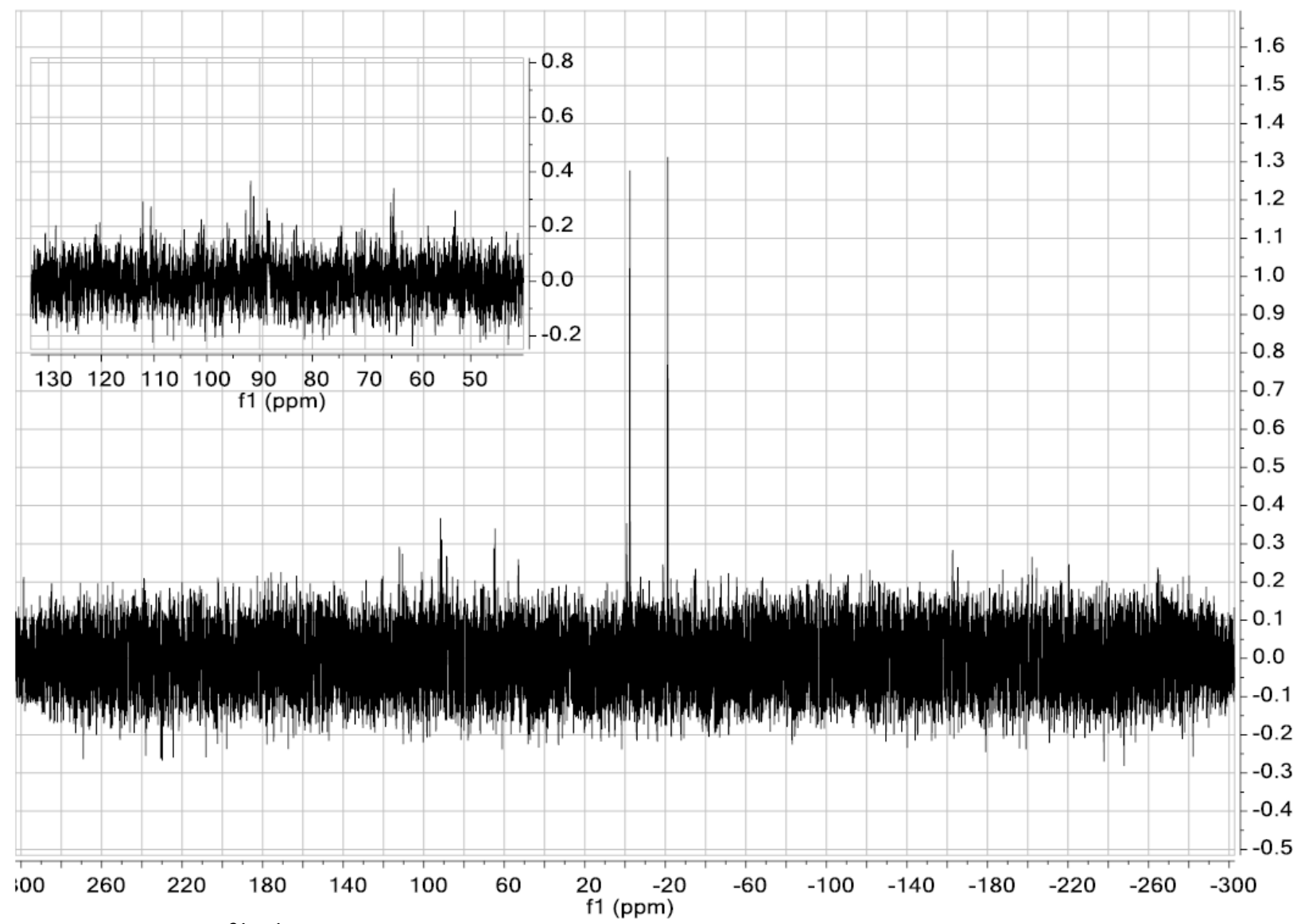

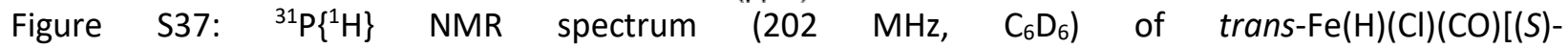
(iPr) $\left.{ }_{2} \mathrm{PCH}_{2} \mathrm{CH}_{2} \mathrm{~N}(\mathrm{H}) \mathrm{CH}\left(\mathrm{CH}\left(\mathrm{CH}_{3}\right)_{2}\right) \mathrm{CH}_{2} \mathrm{P}(\mathrm{Ph})_{2}\right]$ (S)-11. 


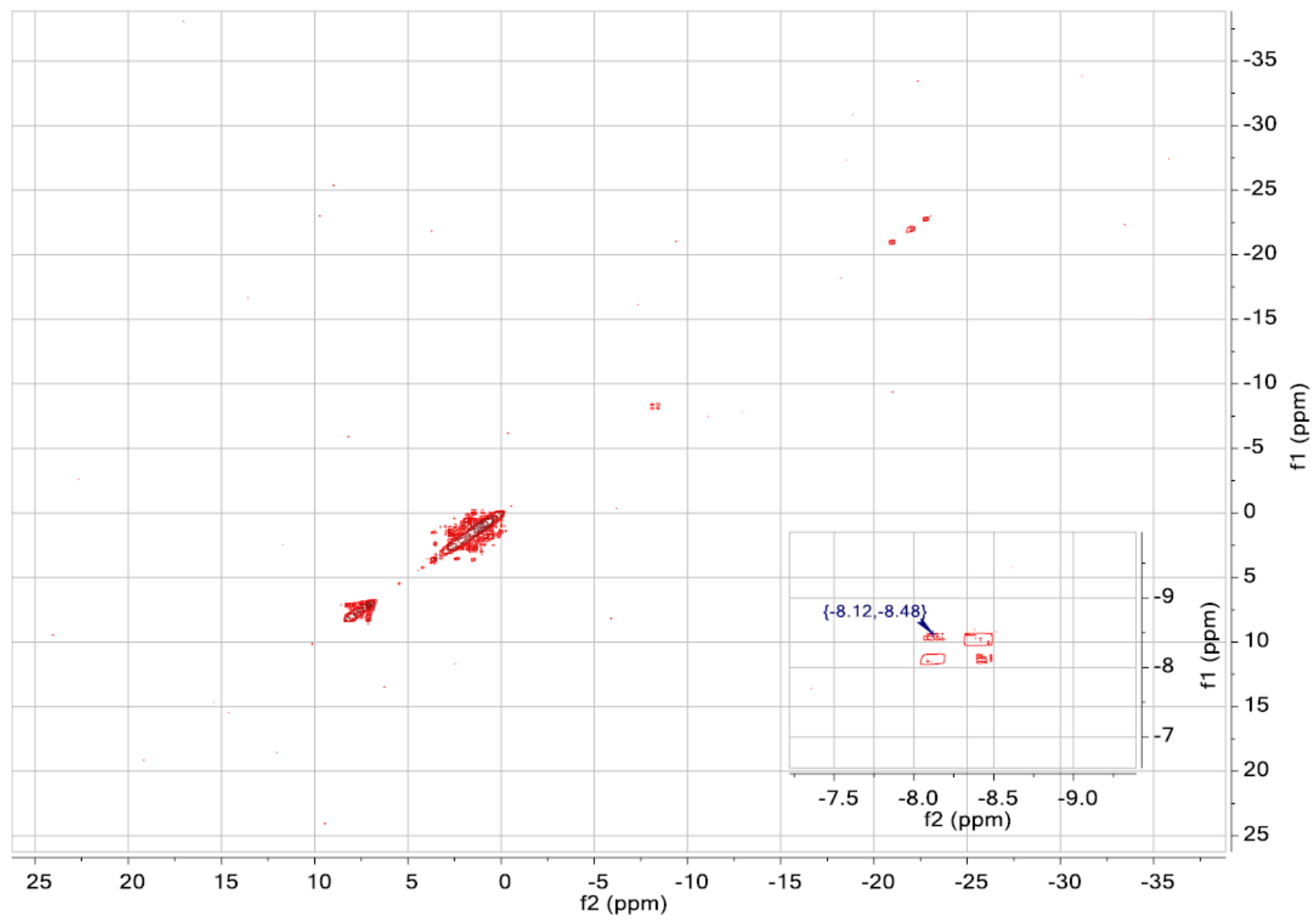

Figure S38: ${ }^{1} \mathrm{H} \quad \mathrm{COSY} \quad \mathrm{NMR}$ spectrum $\left(500 \quad \mathrm{MHz}, \quad \mathrm{C}_{6} \mathrm{D}_{6}\right)$ of trans- $\mathrm{Fe}(\mathrm{H})(\mathrm{Cl})(\mathrm{CO})[(\mathrm{S})-$ (iPr) $\left.{ }_{2} \mathrm{PCH}_{2} \mathrm{CH}_{2} \mathrm{~N}(\mathrm{H}) \mathrm{CH}\left(\mathrm{CH}\left(\mathrm{CH}_{3}\right)_{2}\right) \mathrm{CH}_{2} \mathrm{P}(\mathrm{Ph})_{2}\right]$ (S)-11. 


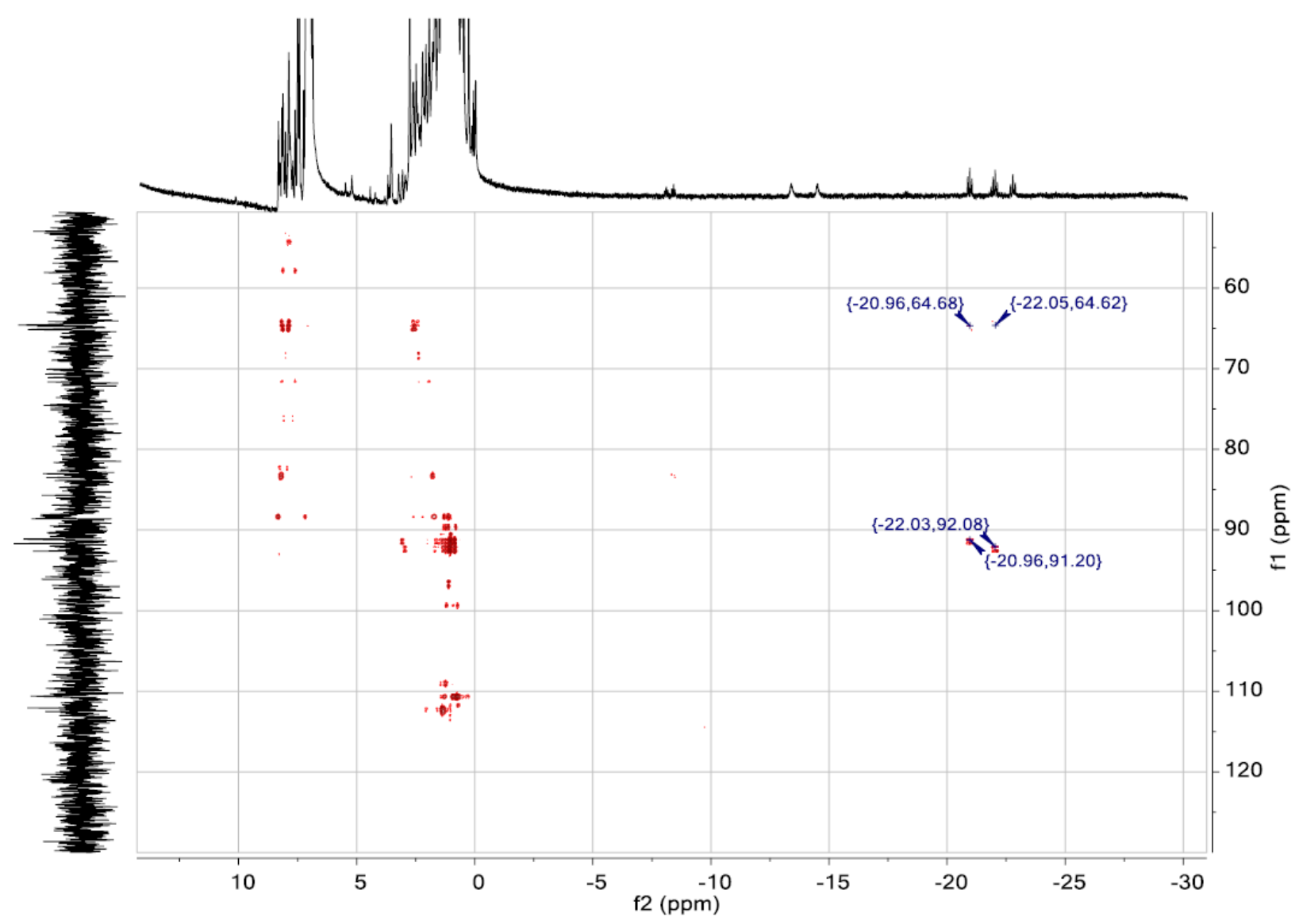

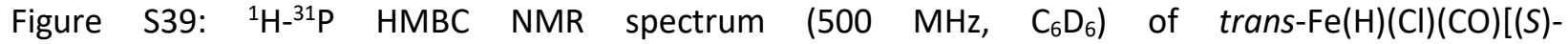
(iPr) $\left.{ }_{2} \mathrm{PCH}_{2} \mathrm{CH}_{2} \mathrm{~N}(\mathrm{H}) \mathrm{CH}\left(\mathrm{CH}\left(\mathrm{CH}_{3}\right)_{2}\right) \mathrm{CH}_{2} \mathrm{P}(\mathrm{Ph})_{2}\right]$ (S)-11.

Catalysis with $\mathrm{Fe}(\mathrm{H})(\mathrm{Cl})(\mathrm{CO})\left[(\mathrm{S}, \mathrm{S})-(\mathrm{Cy})_{2} \mathrm{PCH}_{2} \mathrm{CH}_{2} \mathrm{~N}(\mathrm{H}) \mathrm{CH}(\mathrm{Ph}) \mathrm{CH}(\mathrm{Ph}) \mathrm{P}(\mathrm{Ph})_{2}\right]$ Table S1 Preliminary results for use of catalyst $\mathrm{Fe}(\mathrm{H})(\mathrm{Cl})(\mathrm{CO})\left[(\mathrm{S}, \mathrm{S})-(\mathrm{Cy})_{2} \mathrm{PCH}_{2} \mathrm{CH} \mathrm{H}_{2} \mathrm{~N}(\mathrm{H}) \mathrm{CH}(\mathrm{Ph}) \mathrm{CH}(\mathrm{Ph}) \mathrm{P}(\mathrm{Ph})_{2}{ }^{[a]}\right.$

\begin{tabular}{|l|l|l|l|l|l|}
\hline Entry & $\mathrm{R}^{[\mathrm{b}]}$ & $\mathrm{R}^{[\mathrm{b}]}$ & $\begin{array}{l}\text { Conversion } \\
(\%)^{[\mathrm{c}]}\end{array}$ & $\begin{array}{c}\text { Time } \\
(\min )\end{array}$ & $\begin{array}{c}\text { e.e. }(\mathrm{S})^{[\mathrm{c}]} \\
(\%)\end{array}$ \\
\hline 1 & $\mathrm{CH}_{3}$ & $\mathrm{H}$ & $>99$ & 120 & 91 \\
\hline 2 & $\mathrm{CH}_{2} \mathrm{CH}_{3}$ & $\mathrm{H}$ & $>99$ & 120 & 91 \\
\hline 3 & $\mathrm{CH}_{3}$ & $o-\mathrm{Cl}$ & $>97$ & 120 & 83 \\
\hline 4 & $\mathrm{CH}_{3}$ & $p-\mathrm{Cl}$ & $>99$ & 120 & 83 \\
\hline
\end{tabular}

[a] conditions: 10 bar $\mathrm{H}_{2}, 0.2 \mathrm{~mol} \%$ catalyst, $2 \mathrm{~mol} \% \mathrm{KO}^{\mathrm{t}} \mathrm{Bu}, 50^{\circ} \mathrm{C}, 10 \mathrm{~mL} \mathrm{THF}$; [b] refer to Scheme 4 [c] conversion and e.e. determined by chiral GC. 


\section{Gas Chromatography Traces}

\section{Acetophenone}

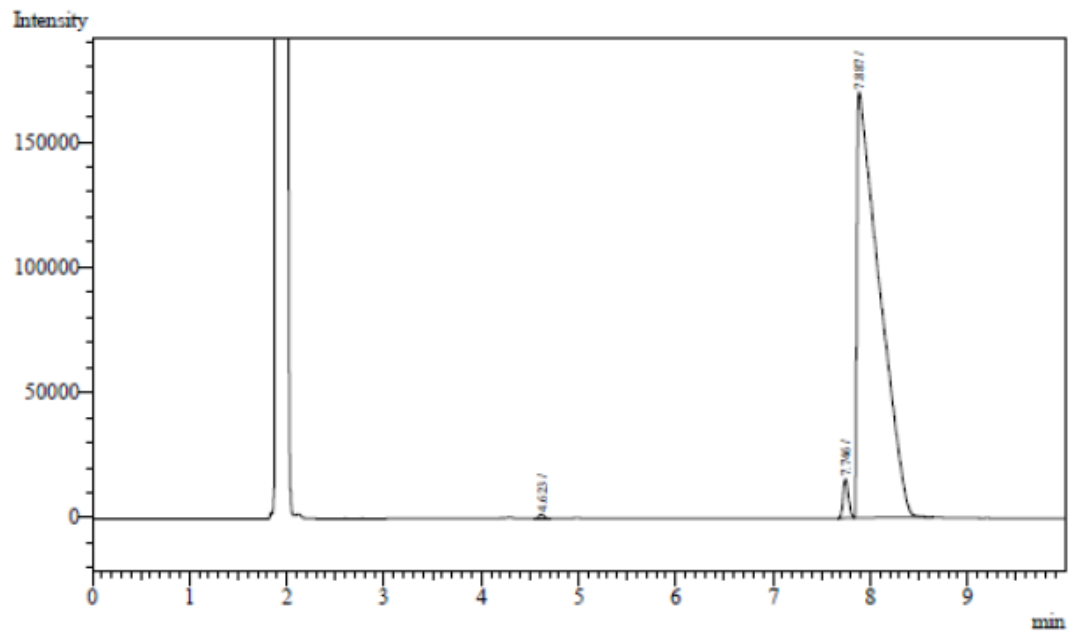

Figure S40: GC trace for entry 1, Table 1

$\mathrm{GC}$ analysis conditions: Oven temperature $130{ }^{\circ} \mathrm{C}$

Retention time: $(R)$-isomer $=6.693 ;(S)$-isomer $=7.193$; starting material $=4.093$.

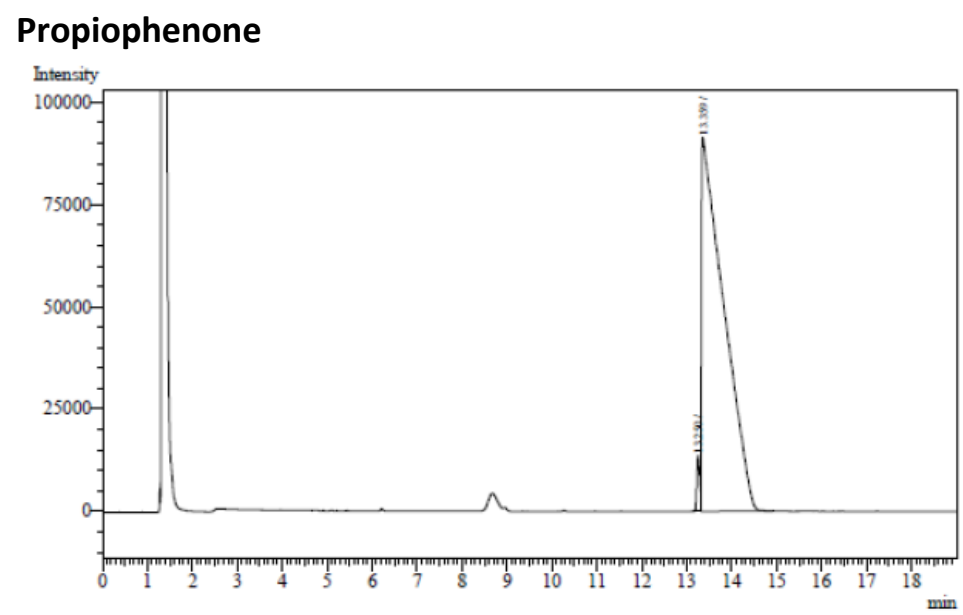

Figure S41: GC trace for entry 2, Table 1

$\mathrm{GC}$ analysis conditions: Oven temperature $120^{\circ} \mathrm{C}$

Retention time: $(R)$-isomer $=10.966 ;(S)$-isomer $=11.992 ;$ starting material $=5.349$ 


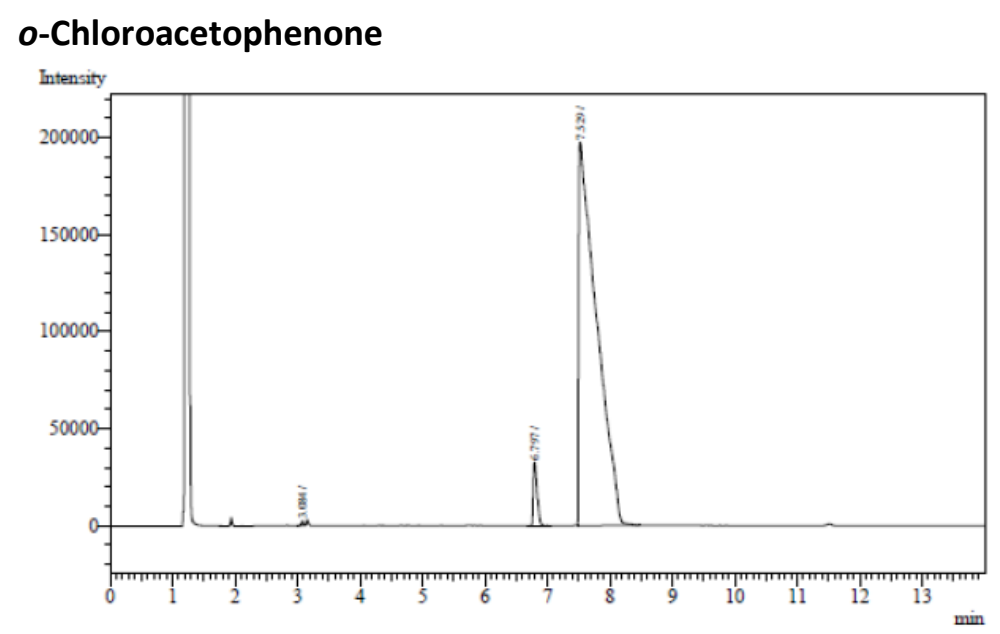

Figure S42: GC trace for entry 3, Table 1

$\mathrm{GC}$ analysis conditions: Oven temperature $145^{\circ} \mathrm{C}$

Retention time: $(R)$-isomer $=5.654 ;(S)$-isomer $=6.924 ;$ starting material $=2.752$.

\section{Cyclohexylphenyl ketone}

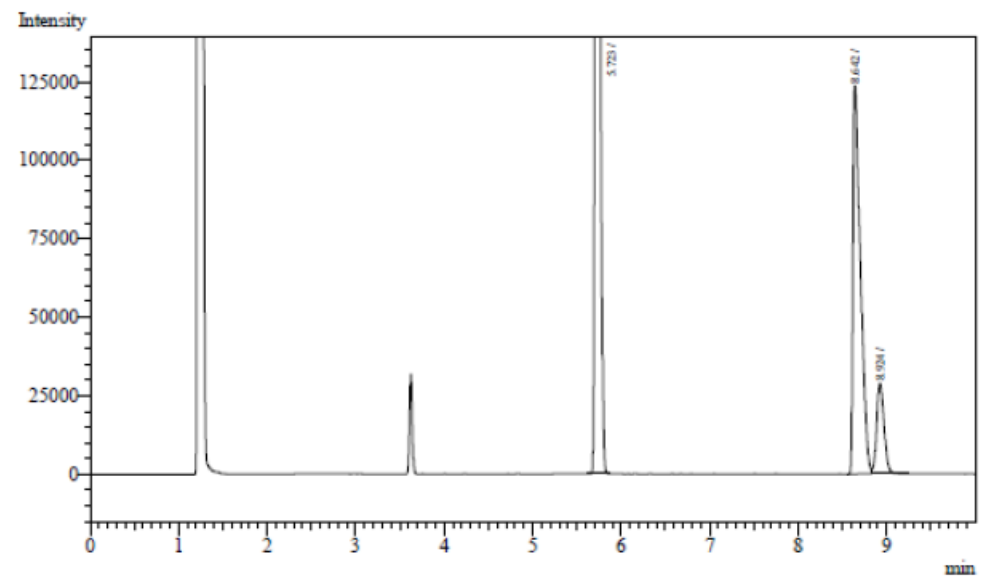

Figure S43: GC trace for entry 4, Table 1

$\mathrm{GC}$ analysis conditions: Oven temperature $170^{\circ} \mathrm{C}$

Retention time: $(R)$-isomer $=8.924 ;(S)$-isomer $=8.642$; starting material $=5.723$. 


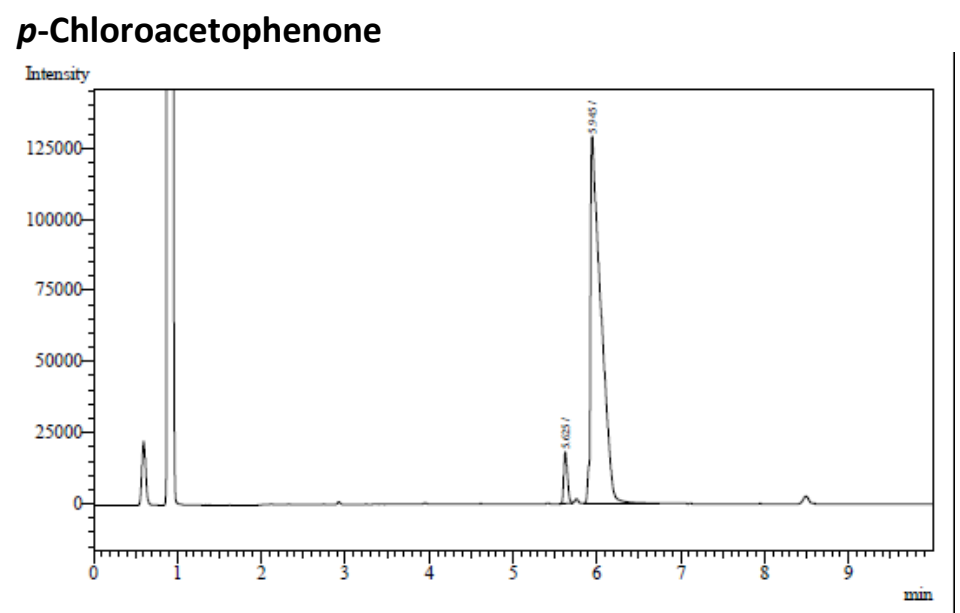

Figure S44: GC trace for entry 6, Table 1

GC analysis conditions: Oven temperature $145^{\circ} \mathrm{C}$

Retention time: $(R)$-isomer $=4.737 ;(S)$-isomer $=5.213$; starting material $=2.588$.

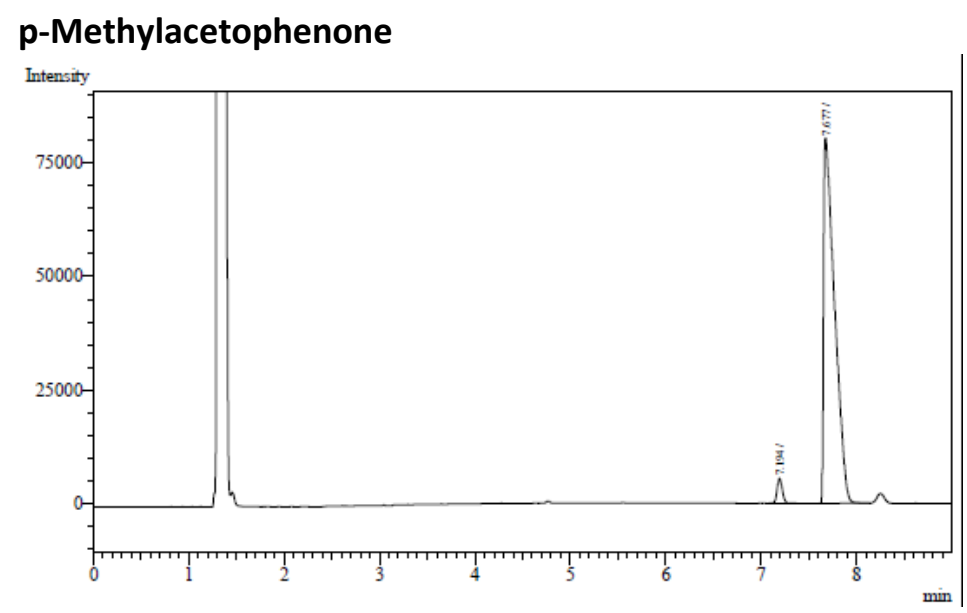

Figure S45: GC trace for entry 7, Table 1

$\mathrm{GC}$ analysis conditions: Oven temperature $130{ }^{\circ} \mathrm{C}$

Retention time: $(R)$-isomer $=7.194 ;(S)$-isomer $=7.677$; starting material $=4.142$. 


\section{2-Acetonaphthone}

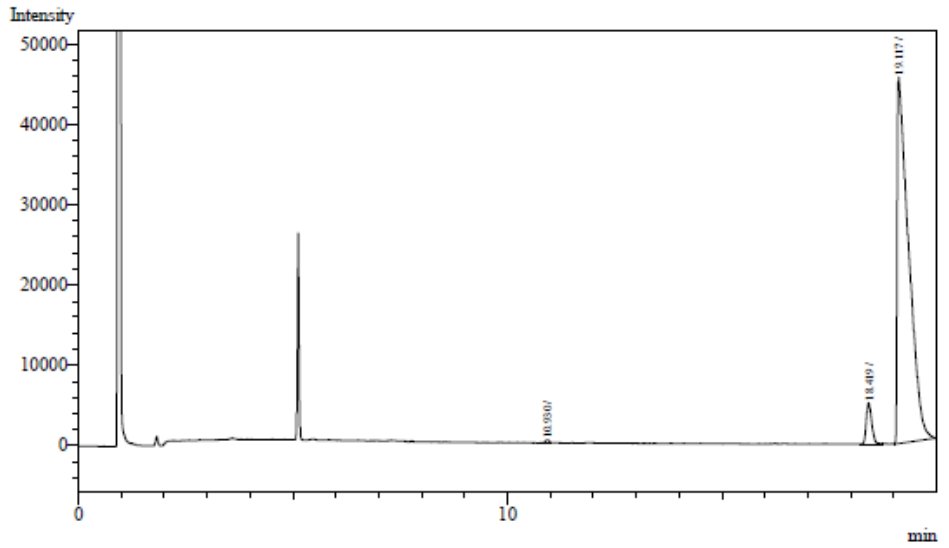

Figure S46: GC trace for entry 1, Table 2

$\mathrm{GC}$ analysis conditions: Oven temperature $150^{\circ} \mathrm{C}$

Retention time: $(R)$-isomer $=6.119 ;(S)$-isomer $=6.378$; starting material $=4.403$.

\section{1-Acetonaphthone}

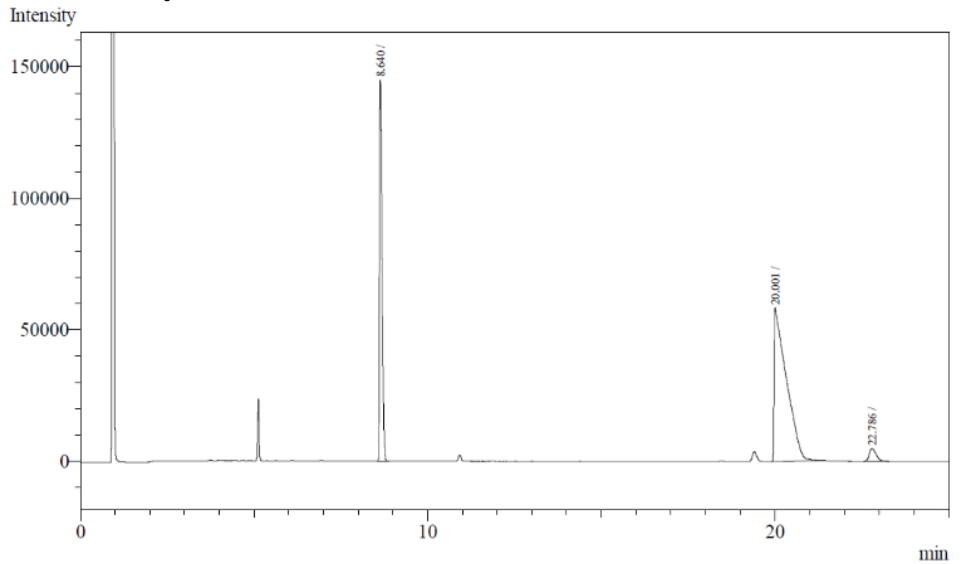

Figure S47: GC trace for entry 2, Table 2

$\mathrm{GC}$ analysis conditions: Oven temperature $150^{\circ} \mathrm{C}$

Retention time: $(R)$-isomer $=22.786 ;(S)$-isomer $=20.001$; starting material $=8.640$.

\section{2-Acetylfuran}

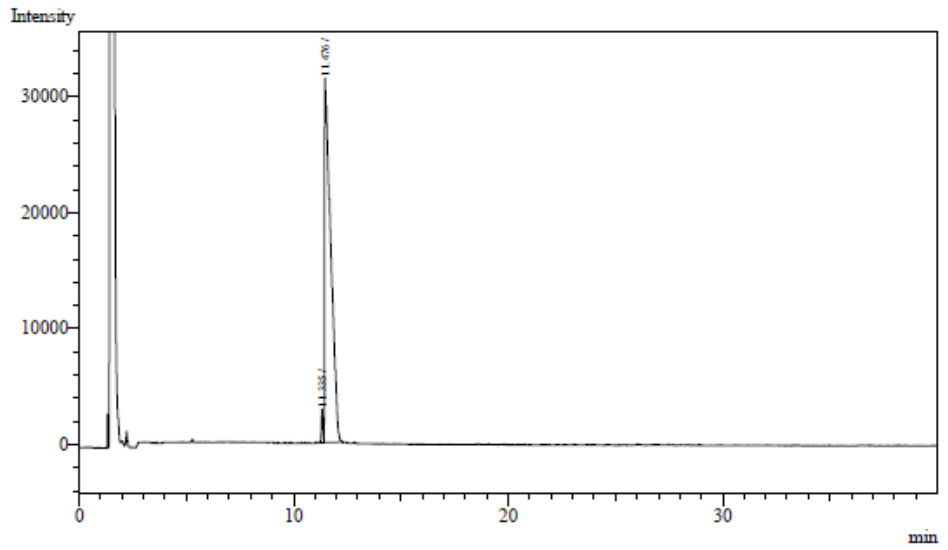

Figure S48: GC trace for entry 3, Table 2 
GC analysis conditions: Oven temperature $90{ }^{\circ} \mathrm{C}$

Retention time: $(R)$-isomer $=11.335 ;(S)$-isomer $=11.476$; starting material $=5.227$.

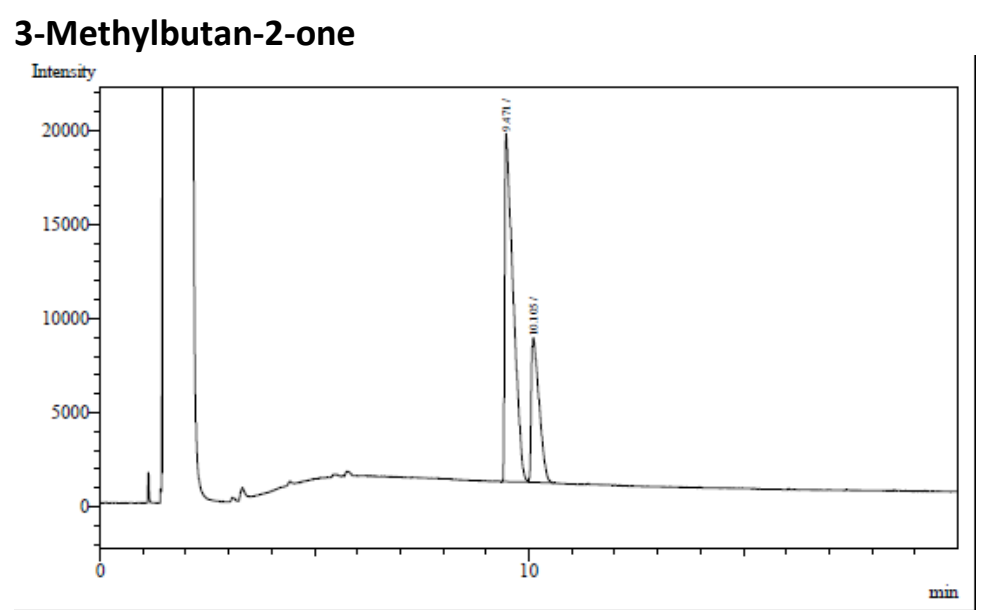

Figure S49: GC trace for entry 4, Table 2

$\mathrm{GC}$ analysis conditions: Oven temperature $50{ }^{\circ} \mathrm{C}$

Retention time: $(R)$-isomer $=9.471 ;(S)$-isomer $=10.105$; starting material $=3.74$. 


\section{DFT calculations}

\section{Calculations}

DFT calculations were performed using Gaussian 09 (Rev. D.01) ${ }^{1}$ at the $M 11 L^{2} / 6-31 G^{*}$ level except SDD for Fe. Normal (opt) convergence criteria were met using a pruned $(99,590)$ integration grid (grid $=$ ultrafine). Optimizations were performed in a THF solvent continuum ( $1 \mathrm{~atm}, 298 \mathrm{~K}$ ) using the integral equation formalism polarizable continuum model (IEF-PCM) ${ }^{3,4}$ with radii and non-electrostatic terms from the SMD solvation model (scrf $=\mathrm{smd}) .{ }^{5}$ Full vibrational and thermochemical analyses were performed on optimized structures to obtain solvent-corrected free energies $\left(G^{\circ}\right)$ and enthalpies $\left(H^{\circ}\right)$. Optimized ground states were found to have zero imaginary frequencies while transition states had one imaginary frequency. Calculations were performed using the facilities of the Shared Hierarchical Academic Research Computing Network (SHARCNET) ${ }^{6}$ and Scinet of Compute/Calcul Canada. ${ }^{7}$

The transition state energies (Figure S50) are higher than those reported for a similar system ${ }^{[8]}$ because of the larger basis set employed in the current work which gives more reliable results. The $S$ transition state is $31.5 \mathrm{kcal} / \mathrm{mol}$ at $323 \mathrm{~K}$ relative to the separate dihydride and ketone species while the $R$-transition state is $33.1 \mathrm{kcal} / \mathrm{mol}$, consistent with the fact that warming to $323 \mathrm{~K}$ is required for reaction. [1] Gaussian 09, Revision or B.01 or D.01, M. J. Frisch, G. W. Trucks, H. B. Schlegel, G. E. Scuseria, M. A. Robb, J. R. Cheeseman, G. Scalmani, V. Barone, B. Mennucci, G. A. Petersson, H. Nakatsuji, M. Caricato, X. Li, H. P. Hratchian, A. F. Izmaylov, J. Bloino, G. Zheng, J. L. Sonnenberg, M. Hada, M. Ehara, K. Toyota, R. Fukuda, J. Hasegawa, M. Ishida, T. Nakajima, Y. Honda, O. Kitao, H. Nakai, T. Vreven, J. A. Montgomery, Jr., J. E. Peralta, F. Ogliaro, M. Bearpark, J. J. Heyd, E. Brothers, K. N. Kudin, V. N. Staroverov, R. Kobayashi, J. Normand, K. Raghavachari, A. Rendell, J. C. Burant, S. S. Iyengar, J. Tomasi, M. Cossi, N. Rega, J. M. Millam, M. Klene, J. E. Knox, J. B. Cross, V. Bakken, C. Adamo, J. Jaramillo, R. Gomperts, R. E. Stratmann, O. Yazyev, A. J. Austin, R. Cammi, C. Pomelli, J. W. Ochterski, R. L. Martin, K. Morokuma, V. G. Zakrzewski, G. A. Voth, P. Salvador, J. J. Dannenberg, S. Dapprich, A. D. Daniels, Ö. Farkas, J. B. Foresman, J. V. Ortiz, J. Cioslowski, and D. J. Fox, Gaussian, Inc., Wallingford CT, 2009.

[2] R. Peverati, D. G. Truhlar, J. Phys. Chem. Lett. 2012, 3, 117-124.

[3] J. Tomasi, B. Mennucci, R. Cammi, Chem. Rev. 2005, 105, 2999-3094.

[4] J. Tomasi, B. Mennucci, E. Cancès, J. Molec. Struct. THEOCHEM 1999, 464, 211-226.

[5] A. V. Marenich, C. J. Cramer, D. G. Truhlar, J. Phys. Chem. B 2009, 113, 6378-6396.

[6] www.sharcnet.ca.

[7] www.computecanada.ca

[8] J. F. Sonnenberg, P. E. Sues, K. Y. Wan, R. H. Morris, ACS Catalysis 2017, 7, 316-326. 
Reaction coordinate diagram for hydride transfer to the ketone.

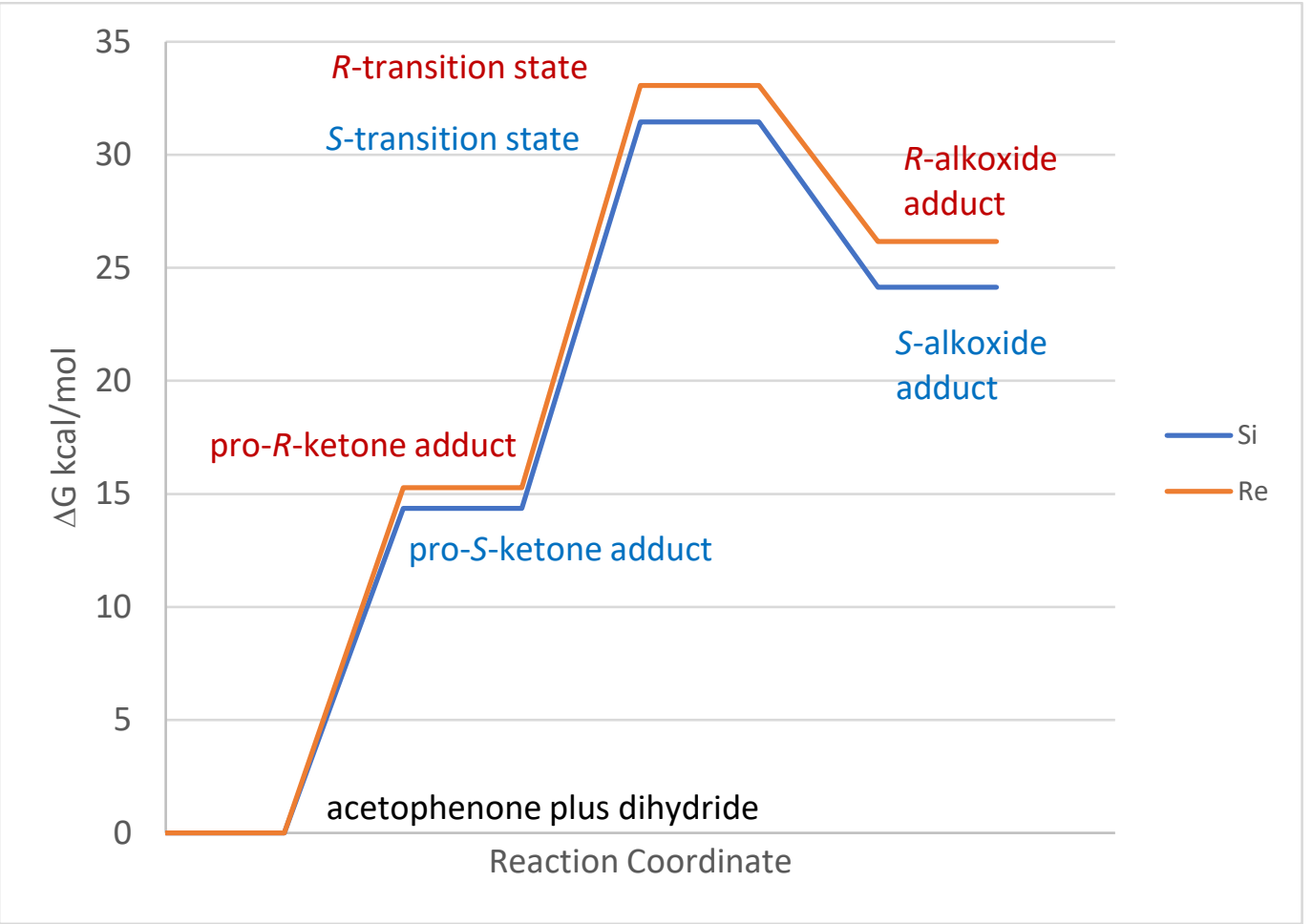

Figure S50. The reaction coordinate diagram for hydride transfer. 


\begin{tabular}{|c|c|c|c|c|}
\hline \multicolumn{5}{|c|}{ Optimized coordinates } \\
\hline \multicolumn{5}{|c|}{ Acetophenone } \\
\hline \multicolumn{5}{|c|}{ delta G (kcal/mol) (323) -241401.3464 } \\
\hline 6 & -0.207422000 & -0.053000000 & -0.000162000 & \\
\hline 6 & 0.426003000 & 1.189114000 & -0.000325000 & \\
\hline 6 & 0.569004000 & -1.211624000 & 0.000189000 & \\
\hline 6 & -1.690822000 & -0.204317000 & -0.000127000 & \\
\hline 6 & 1.809017000 & 1.268752000 & -0.000245000 & \\
\hline 1 & -0.162729000 & 2.113871000 & -0.000485000 & \\
\hline 6 & 1.948590000 & -1.132614000 & 0.000317000 & \\
\hline 1 & 0.058526000 & -2.181271000 & 0.000424000 & \\
\hline 6 & 2.570769000 & 0.109459000 & 0.000065000 & \\
\hline 1 & 2.300023000 & 2.248267000 & -0.000396000 & \\
\hline 1 & 2.551794000 & -2.047548000 & 0.000656000 & \\
\hline 1 & 3.665039000 & 0.174099000 & 0.000174000 & \\
\hline 6 & -2.530531000 & 1.036496000 & 0.000568000 & \\
\hline 8 & -2.192703000 & -1.300344000 & -0.000541000 & \\
\hline 1 & -2.322546000 & 1.661188000 & 0.885242000 & \\
\hline 1 & -3.593124000 & 0.758558000 & 0.000678000 & \\
\hline 1 & -2.322995000 & 1.661991000 & -0.883648000 & \\
\hline \multicolumn{5}{|c|}{ Dihydride trans-FeH ${ }_{2}(\mathrm{CO})($ mer- $(S, S)-1)$} \\
\hline del & $\mathrm{H}$ (Hartrees) & -2295.234072 & 2 delta S (cal/mol/K) & 241.77 \\
\hline \multicolumn{5}{|c|}{ delta G (kcal/mol) (323) -1440337.472 } \\
\hline 1 & -5.841578000 & 0.090269000 & -1.889635000 & \\
\hline 1 & -5.124188000 & 1.365536000 & 2.150778000 & \\
\hline 1 & 3.745371000 & -1.268508000 & 2.513200000 & \\
\hline 6 & 4.713973000 & -0.883797000 & 2.149781000 & \\
\hline 6 & -0.555563000 & 1.665151000 & 0.042374000 & \\
\hline 15 & -0.607705000 & -1.035001000 & -0.052268000 & \\
\hline 6 & 3.122113000 & 2.173429000 & -0.355123000 & \\
\hline 1 & -0.939575000 & -3.892916000 & 0.149114000 & \\
\hline 1 & -1.659521000 & -2.726122000 & -4.610898000 & \\
\hline 6 & -3.386780000 & 1.042856000 & 0.933240000 & \\
\hline 7 & 0.829319000 & 1.428506000 & -0.372988000 & \\
\hline 1 & -3.305196000 & -2.221854000 & 0.264714000 & \\
\hline 6 & -1.801862000 & -1.754702000 & 3.793067000 & \\
\hline 1 & -0.039366000 & -1.019793000 & 2.793117000 & \\
\hline 6 & -5.638525000 & 0.725644000 & 0.159020000 & \\
\hline 6 & 2.031466000 & -2.090568000 & 0.402377000 & \\
\hline 1 & 1.601705000 & -0.708875000 & -1.508710000 & \\
\hline 1 & -1.219764000 & -0.948278000 & -2.987768000 & \\
\hline 6 & 4.550841000 & 0.496168000 & 1.549834000 & \\
\hline 6 & -1.576655000 & -1.574505000 & 1.396285000 & \\
\hline 6 & -1.269388000 & -1.983701000 & -2.634343000 & \\
\hline 6 & -3.082100000 & -2.258091000 & 3.645788000 & \\
\hline 6 & 1.725626000 & 2.464139000 & 0.123899000 & \\
\hline
\end{tabular}




$\begin{array}{lccc}1 & -1.372660000 & -1.625475000 & 4.792325000 \\ 1 & -3.674065000 & -2.524760000 & 4.527802000 \\ 6 & -4.750257000 & 1.072117000 & 1.164287000 \\ 26 & 1.502735000 & -0.469100000 & 0.042661000 \\ 1 & 4.651812000 & 1.426923000 & 3.486940000 \\ 15 & 3.509769000 & 0.429559000 & 0.021986000 \\ 1 & 1.664670000 & 2.441468000 & 1.227295000 \\ 1 & -6.717294000 & 0.746760000 & 0.344604000 \\ 1 & 5.396795000 & -0.848210000 & 3.016024000 \\ 6 & -3.785437000 & 0.336226000 & -1.314892000 \\ 1 & 3.941268000 & 2.484650000 & 2.257142000 \\ 1 & -1.256992000 & 0.611841000 & -1.665388000 \\ 6 & 3.998903000 & 1.438309000 & 2.597068000 \\ 6 & -1.053454000 & -1.416826000 & 2.675805000 \\ 1 & 5.546602000 & 0.875435000 & 1.244962000 \\ 6 & -2.866550000 & -2.091822000 & 1.261210000 \\ 6 & -2.881723000 & 0.669848000 & -0.310321000 \\ 1 & -3.406400000 & 0.047088000 & -2.302964000 \\ 8 & 2.349533000 & -3.170375000 & 0.642525000 \\ 6 & -1.414496000 & 0.559875000 & -0.569707000 \\ 6 & -1.082334000 & -2.283407000 & -1.287481000 \\ 6 & -5.150269000 & 0.358465000 & -1.083825000 \\ 1 & -0.551044000 & 1.570384000 & 1.147803000 \\ 6 & -1.324510000 & -4.627871000 & -1.827662000 \\ 1 & -1.717277000 & -5.105696000 & -3.892034000 \\ 1 & 1.404798000 & 3.471599000 & -0.203805000 \\ 1 & 2.992792000 & 1.129631000 & 2.927371000 \\ 1 & 3.162052000 & 2.294170000 & -1.454865000 \\ 1 & -1.333891000 & -5.672513000 & -1.499791000 \\ 6 & -1.535440000 & -4.312800000 & -3.159484000 \\ 6 & -1.101083000 & -3.623838000 & -0.901600000 \\ 1 & 5.120947000 & -1.625568000 & 1.444760000 \\ 1 & -2.702267000 & 1.292040000 & 1.752701000 \\ 6 & -1.504106000 & -2.987325000 & -3.558882000 \\ 1 & -4.624899000 & -2.825261000 & 2.250094000 \\ 1 & 3.843052000 & 2.896728000 & 0.061510000 \\ 6 & -3.612029000 & -2.428548000 & 2.375559000 \\ 6 & 4.675613000 & 0.015964000 & -1.352154000 \\ 1 & 6.654956000 & 0.503294000 & -0.583374000 \\ 6 & 4.969825000 & -1.461005000 & -1.477174000 \\ 6 & 5.951613000 & 0.826673000 & -1.369224000 \\ 1 & 4.051688000 & -2.070444000 & -1.487286000 \\ 1 & 4.071043000 & 0.303593000 & -2.237414000 \\ 1 & 5.507430000 & -1.663621000 & -2.419275000 \\ 1 & 5.614601000 & -1.827301000 & -0.660898000 \\ 1 & 5.779404000 & 1.908411000 & -1.239532000 \\ 1 & 6.474569000 & 0.695356000 & -2.332195000 \\ 1 & 0.852670000 & 1.476562000 & -1.396510000\end{array}$




$\begin{array}{rrrr}6 & -1.066635000 & 3.037659000 & -0.317991000 \\ 6 & -1.441122000 & 3.932226000 & 0.675275000 \\ 6 & -1.155044000 & 3.439591000 & -1.646653000 \\ 6 & -1.901687000 & 5.198397000 & 0.353114000 \\ 6 & -1.609116000 & 4.705310000 & -1.972493000 \\ 6 & -1.986714000 & 5.588046000 & -0.972572000 \\ 1 & -1.367254000 & 3.626304000 & 1.726470000 \\ 1 & -0.858364000 & 2.752970000 & -2.450110000 \\ 1 & -2.195187000 & 5.890331000 & 1.149336000 \\ 1 & -1.671605000 & 5.007048000 & -3.023304000 \\ 1 & -2.348967000 & 6.588289000 & -1.231027000 \\ 1 & 1.403013000 & -0.083520000 & 1.546063000\end{array}$

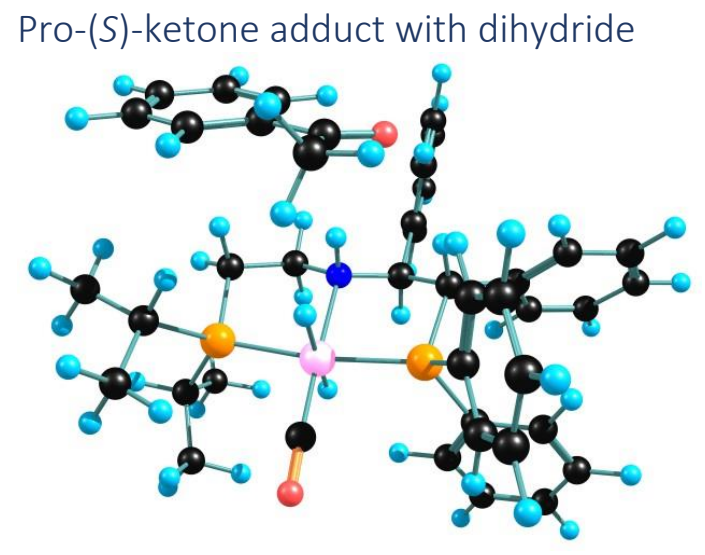

$\begin{array}{lccc}\text { delta H (Hartrees) } & -2679.89 & \text { delta S (cal/m } \\ \text { delta G (kcal/mol) }(323) & -1681723.575 & \\ 1 & 5.343978000 & 2.733402000 & 2.035293000 \\ 1 & 5.495689000 & 1.967950000 & -2.190564000 \\ 1 & -1.345534000 & -3.687000000 & -2.775977000 \\ 6 & -2.445775000 & -3.746444000 & -2.716584000 \\ 6 & 0.646065000 & 1.211722000 & -1.088121000 \\ 15 & 1.458954000 & -0.978613000 & 0.259315000 \\ 6 & -2.962049000 & 0.308673000 & -1.481482000 \\ 1 & 2.696516000 & -3.292039000 & 1.478523000 \\ 1 & 1.472031000 & -0.228896000 & 5.172892000 \\ 6 & 3.654932000 & 1.477612000 & -1.201345000 \\ 7 & -0.670435000 & 0.639074000 & -0.784010000 \\ 1 & 4.345616000 & -0.994231000 & 0.919389000 \\ 6 & 3.932413000 & -2.592912000 & -2.573180000 \\ 1 & 1.813194000 & -2.307145000 & -2.300028000 \\ 6 & 5.570460000 & 2.396640000 & -0.080758000 \\ 6 & -0.454151000 & -3.093385000 & -0.087826000 \\ 1 & -1.105621000 & -1.093386000 & 1.049124000 \\ 1 & 1.041968000 & 0.445217000 & 2.860685000 \\ 6 & -3.058135000 & -2.360555000 & -2.718618000 \\ 6 & 2.938087000 & -1.599725000 & -0.610153000 \\ 6 & 1.520479000 & -0.522734000 & 3.046388000\end{array}$




$\begin{array}{lccc}6 & 5.194179000 & -2.432286000 & -2.028935000 \\ 6 & -1.657388000 & 0.987574000 & -1.797474000 \\ 1 & 3.812945000 & -3.049703000 & -3.561367000 \\ 1 & 6.079849000 & -2.756013000 & -2.585997000 \\ 6 & 4.953788000 & 1.951743000 & -1.239145000 \\ 26 & -0.584125000 & -1.387089000 & -0.408338000 \\ 1 & -2.865176000 & -2.214016000 & -4.858198000 \\ 15 & -2.650816000 & -1.457543000 & -1.154446000 \\ 1 & -1.254985000 & 0.652131000 & -2.771287000 \\ 1 & 6.599816000 & 2.768368000 & -0.111609000 \\ 1 & -2.791759000 & -4.318001000 & -3.595032000 \\ 6 & 3.569159000 & 1.905981000 & 1.149321000 \\ 1 & -3.096195000 & -0.638308000 & -4.082587000 \\ 1 & 1.082286000 & 1.268206000 & 0.993024000 \\ 6 & -2.614223000 & -1.621382000 & -3.961420000 \\ 6 & 2.811824000 & -2.180824000 & -1.867871000 \\ 1 & -4.161632000 & -2.461343000 & -2.756565000 \\ 6 & 4.216681000 & -1.452903000 & -0.068219000 \\ 6 & 2.943975000 & 1.441537000 & -0.003939000 \\ 1 & 3.017930000 & 1.895784000 & 2.097917000 \\ 8 & -0.335495000 & -4.216556000 & 0.136906000 \\ 6 & 1.567387000 & 0.868903000 & 0.079928000 \\ 6 & 1.875919000 & -1.357569000 & 1.989466000 \\ 6 & 4.871638000 & 2.374479000 & 1.114786000 \\ 1 & 0.985021000 & 0.683474000 & -2.002976000 \\ 6 & 2.635918000 & -2.993564000 & 3.599958000 \\ 1 & 2.477713000 & -2.445433000 & 5.678560000 \\ 1 & -1.800760000 & 2.084406000 & -1.861406000 \\ 1 & -1.521071000 & -1.470934000 & -3.969187000 \\ 1 & -3.372808000 & 0.732106000 & -0.545845000 \\ 1 & 3.068909000 & -3.977588000 & 3.808416000 \\ 6 & 2.304400000 & -2.142584000 & 4.640864000 \\ 6 & 2.424334000 & -2.604816000 & 2.288422000 \\ 1 & -2.694349000 & -4.339318000 & -1.822263000 \\ 1 & 3.196160000 & 1.104193000 & -2.124840000 \\ 6 & 1.744045000 & -0.908486000 & 4.357561000 \\ 1 & 6.328340000 & -1.731575000 & -0.333999000 \\ 1 & -3.711690000 & 0.507844000 & -2.265780000 \\ 6 & 5.333321000 & -1.863280000 & -0.771767000 \\ 6 & -4.041951000 & -1.858259000 & -0.006040000 \\ 1 & -5.753660000 & -2.112946000 & -1.337159000 \\ 6 & -4.030101000 & -3.293991000 & 0.464470000 \\ 6 & -5.415586000 & -1.470584000 & -0.506149000 \\ 1 & -3.041749000 & -3.608201000 & 0.838853000 \\ 1 & -3.798555000 & -1.218185000 & 0.867485000 \\ 1 & -4.750388000 & -3.433427000 & 1.288705000 \\ 1 & -4.328796000 & -3.991143000 & -0.337087000 \\ 1 & -5.474998000 & -0.423839000 & -0.846985000\end{array}$




$\begin{array}{lrrr}1 & -6.159705000 & -1.589643000 & 0.301280000 \\ 1 & -0.987152000 & 1.056398000 & 0.098273000 \\ 6 & 0.619567000 & 2.690898000 & -1.382527000 \\ 6 & 0.824953000 & 3.143968000 & -2.679208000 \\ 6 & 0.375532000 & 3.624019000 & -0.379431000 \\ 6 & 0.782201000 & 4.496543000 & -2.975309000 \\ 6 & 0.328180000 & 4.976107000 & -0.673250000 \\ 6 & 0.530894000 & 5.416887000 & -1.971813000 \\ 1 & 1.023377000 & 2.415279000 & -3.475528000 \\ 1 & 0.219480000 & 3.292754000 & 0.655357000 \\ 1 & 0.948443000 & 4.835282000 & -4.003304000 \\ 1 & 0.138323000 & 5.698567000 & 0.127850000 \\ 1 & 0.498001000 & 6.486997000 & -2.201045000 \\ 1 & -0.125731000 & -1.537767000 & -1.886265000 \\ 6 & -3.023975000 & 1.932432000 & 2.144526000 \\ 6 & -4.204752000 & 1.212116000 & 2.311974000 \\ 6 & -2.992348000 & 2.978680000 & 1.223059000 \\ 6 & -1.787877000 & 1.630343000 & 2.915337000 \\ 6 & -5.327843000 & 1.529396000 & 1.567490000 \\ 1 & -4.251167000 & 0.383781000 & 3.026956000 \\ 6 & -4.114395000 & 3.298319000 & 0.483535000 \\ 1 & -2.066015000 & 3.551286000 & 1.106765000 \\ 6 & -5.284461000 & 2.571647000 & 0.654400000 \\ 1 & -6.251886000 & 0.958024000 & 1.703852000 \\ 1 & -4.081280000 & 4.126264000 & -0.231908000 \\ 1 & -6.176237000 & 2.824459000 & 0.071260000 \\ 6 & -1.857703000 & 0.612279000 & 4.004151000 \\ 8 & -0.755465000 & 2.205436000 & 2.658234000 \\ 1 & -2.710292000 & 0.783790000 & 4.679059000 \\ 1 & -0.925549000 & 0.628528000 & 4.583688000 \\ 1 & -1.980624000 & -0.395585000 & 3.571861000\end{array}$

(S)-transition state

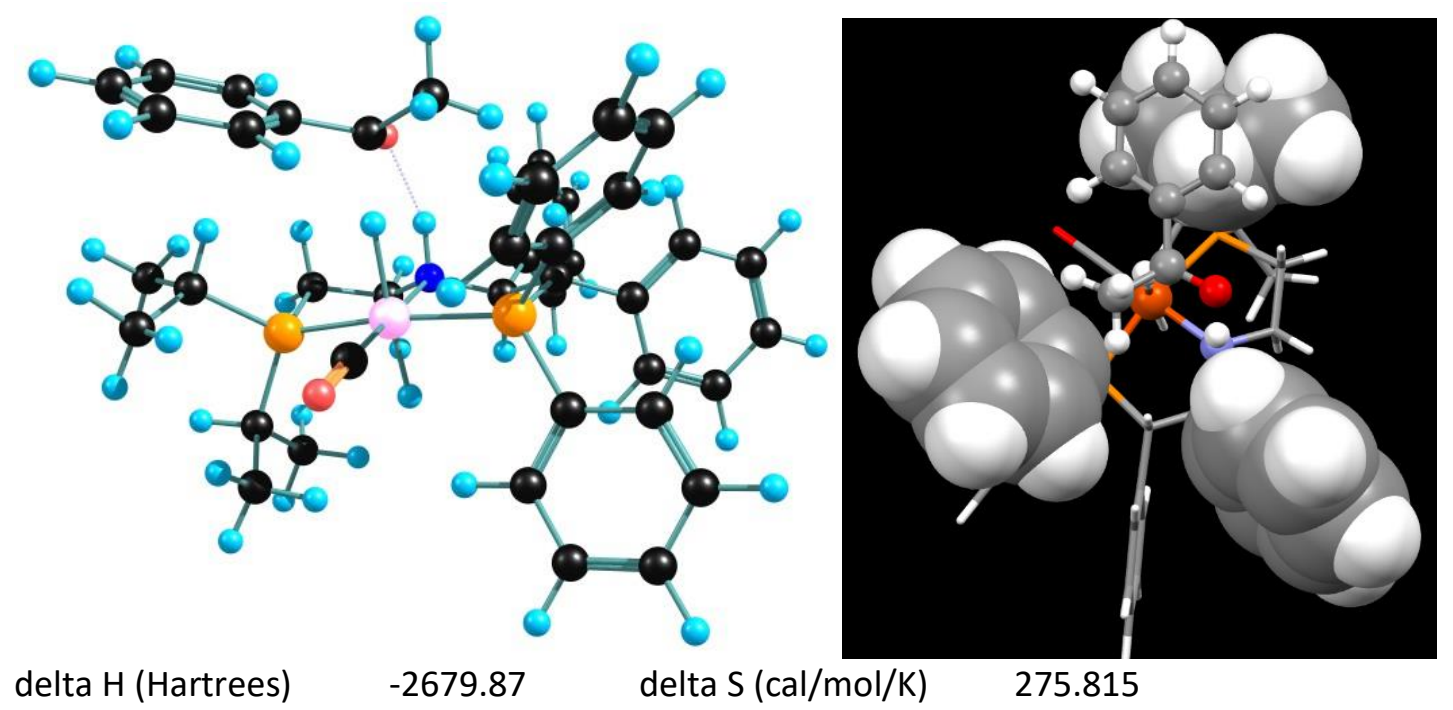




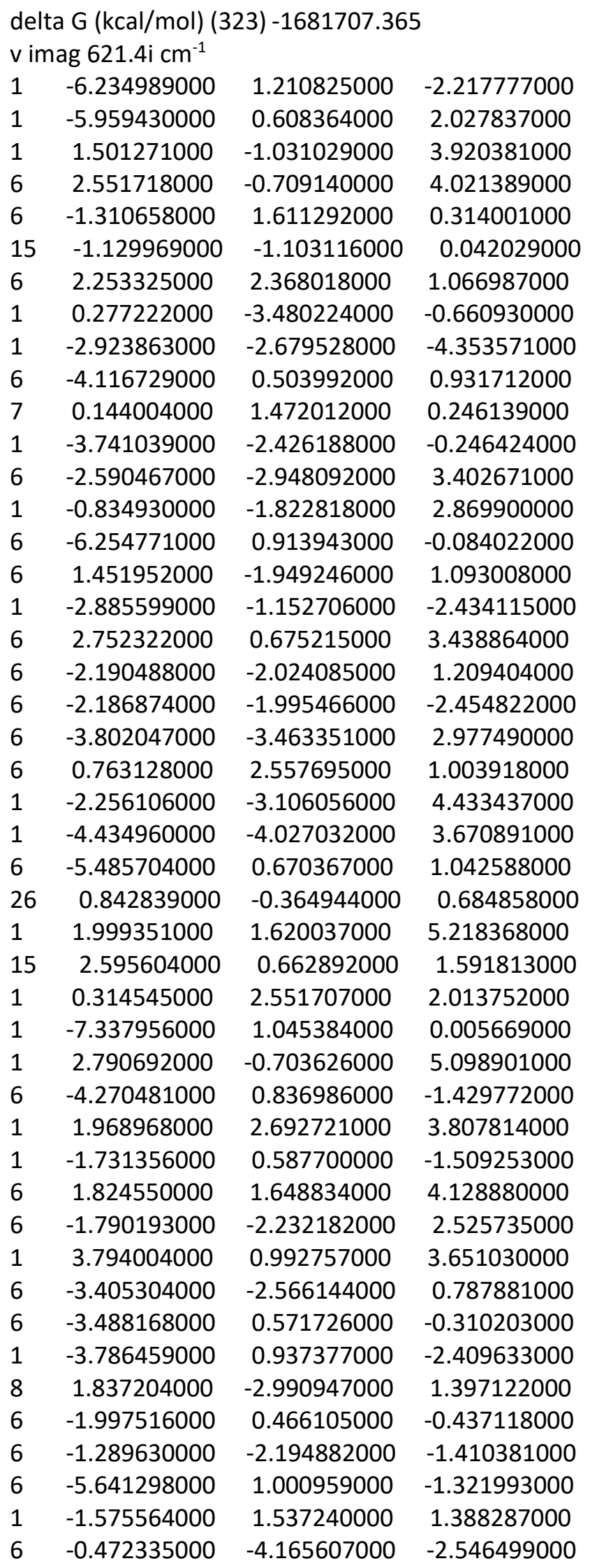




\begin{tabular}{rrrr}
1 & -1.376760000 & -4.621890000 & -4.449101000 \\
1 & 0.522804000 & 3.539300000 & 0.552607000 \\
1 & 0.765867000 & 1.382331000 & 3.966858000 \\
1 & 2.670407000 & 2.468663000 & 0.046157000 \\
1 & 0.208550000 & -5.022738000 & -2.572933000 \\
6 & -1.355724000 & -3.942890000 & -3.590536000 \\
6 & -0.438518000 & -3.296753000 & -1.470568000 \\
1 & 3.177713000 & -1.483886000 & 3.552741000 \\
1 & -3.525071000 & 0.311880000 & 1.834978000 \\
6 & -2.214717000 & -2.858709000 & -3.538551000 \\
1 & -5.162206000 & -3.679313000 & 1.318359000 \\
1 & 2.727112000 & 3.146693000 & 1.688879000 \\
6 & -4.207508000 & -3.270968000 & 1.665663000 \\
6 & 4.336985000 & 0.406918000 & 1.036401000 \\
1 & 5.369495000 & 1.795182000 & 2.382014000 \\
6 & 4.943057000 & -0.879751000 & 1.548502000 \\
6 & 5.255852000 & 1.577795000 & 1.305471000 \\
1 & 4.305987000 & -1.758764000 & 1.353775000 \\
1 & 4.236867000 & 0.307754000 & -0.055834000 \\
1 & 5.908602000 & -1.062823000 & 1.045760000 \\
1 & 5.151255000 & -0.842316000 & 2.632116000 \\
1 & 4.924931000 & 2.503862000 & 0.809047000 \\
1 & 6.265870000 & 1.352584000 & 0.919172000 \\
1 & 0.447812000 & 1.599909000 & -0.734624000 \\
6 & -1.815058000 & 2.932921000 & -0.214040000 \\
6 & -2.666971000 & 3.721022000 & 0.547038000 \\
6 & -1.469719000 & 3.357876000 & -1.493783000 \\
6 & -3.169709000 & 4.909959000 & 0.044173000 \\
6 & -1.964272000 & 4.549673000 & -1.994077000 \\
6 & -2.818645000 & 5.327748000 & -1.228188000 \\
1 & -2.946937000 & 3.390331000 & 1.555048000 \\
1 & -0.787814000 & 2.753587000 & -2.108115000 \\
1 & -3.843234000 & 5.518698000 & 0.656586000 \\
1 & -1.678507000 & 4.876027000 & -2.999737000 \\
1 & -3.212378000 & 6.268225000 & -1.627282000 \\
1 & 0.204076000 & -0.181747000 & 2.063130000 \\
1 & 1.453979000 & -0.336600000 & -0.864764000 \\
6 & 1.803029000 & 0.409137000 & -2.282479000 \\
6 & 3.268856000 & 0.054450000 & -2.277824000 \\
6 & 3.735622000 & -1.245618000 & -2.108602000 \\
6 & 4.186520000 & 1.081274000 & -2.462607000 \\
6 & 5.093175000 & -1.508027000 & -2.099230000 \\
1 & 3.021482000 & -2.060188000 & -1.929740000 \\
6 & 5.547822000 & 0.820205000 & -2.458185000 \\
1 & 3.806994000 & 2.100090000 & -2.595358000 \\
1 & 6.004933000 & -0.473921000 & -2.269620000 \\
& 6.450260000 & -2.532160000 & -1.944991000 \\
\hline
\end{tabular}




$\begin{array}{lrrr}1 & 7.079943000 & -0.682837000 & -2.256775000 \\ 6 & 0.951562000 & -0.456872000 & -3.177836000 \\ 8 & 1.494970000 & 1.608784000 & -2.175251000 \\ 1 & 1.097213000 & -1.539342000 & -3.053950000 \\ 1 & 1.211894000 & -0.197506000 & -4.220866000 \\ 1 & -0.111506000 & -0.215878000 & -3.034547000\end{array}$

(S)-alkoxide adduct

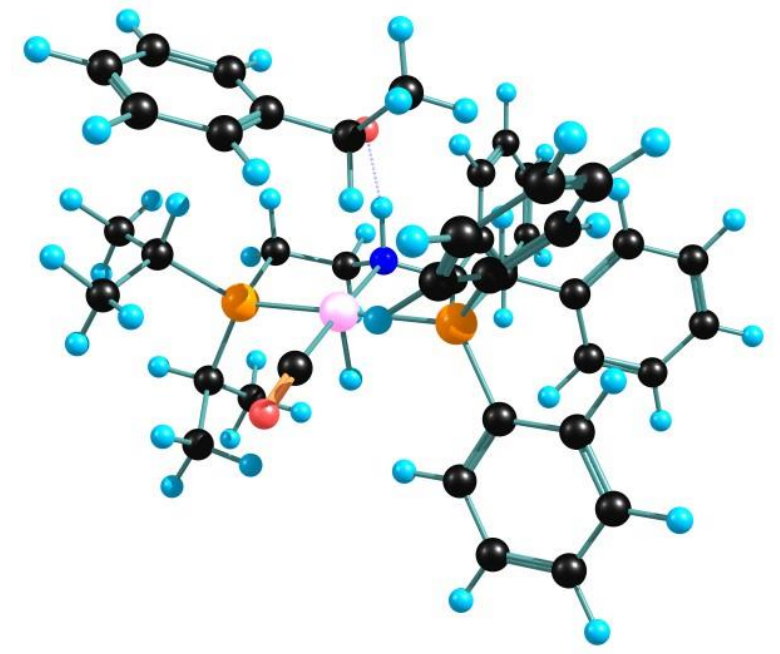

$\begin{array}{lccc}\text { delta H (Hartrees) } & -2679.88 & \text { delta S (cal/mol/K) } & 278.422 \\ \text { delta G (kcal/mol) } & (323)-1681714.683 & \\ 1 & -6.057904000 & 1.209971000 & -2.447935000 \\ 1 & -6.048486000 & 0.591215000 & 1.804366000 \\ 1 & 1.697738000 & -1.068060000 & 3.984319000 \\ 6 & 2.747281000 & -0.732360000 & 4.036446000 \\ 6 & -1.273252000 & 1.568557000 & 0.382380000 \\ 15 & -1.123730000 & -1.133971000 & 0.098101000 \\ 6 & 2.291798000 & 2.344208000 & 1.098543000 \\ 1 & 0.472866000 & -3.402553000 & -0.649261000 \\ 1 & -2.796546000 & -2.749616000 & -4.312288000 \\ 6 & -4.141441000 & 0.485454000 & 0.824738000 \\ 7 & 0.182390000 & 1.425874000 & 0.322703000 \\ 1 & -3.647920000 & -2.596114000 & -0.273227000 \\ 6 & -2.755880000 & -2.753673000 & 3.482423000 \\ 1 & -0.996533000 & -1.622944000 & 2.978089000 \\ 6 & -6.211630000 & 0.903919000 & -0.320708000 \\ 6 & 1.428524000 & -1.989967000 & 1.270311000 \\ 1 & -2.847521000 & -1.256229000 & -2.364961000 \\ 6 & 2.903229000 & 0.653618000 & 3.445798000 \\ 6 & -2.219495000 & -2.020075000 & 1.248289000 \\ 6 & -2.096659000 & -2.051833000 & -2.405687000 \\ 6 & -3.915831000 & -3.348900000 & 3.018374000 \\ 6 & 0.797060000 & 2.510187000 & 1.083793000 \\ 1 & -2.497682000 & -2.805274000 & 4.545252000\end{array}$




$\begin{array}{lccc}1 & -4.582375000 & -3.870674000 & 3.712917000 \\ 6 & -5.514644000 & 0.654411000 & 0.850406000 \\ 26 & 0.861758000 & -0.395830000 & 0.783942000 \\ 1 & 2.206147000 & 1.590056000 & 5.250027000 \\ 15 & 2.674870000 & 0.646356000 & 1.609202000 \\ 1 & 0.374723000 & 2.495505000 & 2.105733000 \\ 1 & -7.297993000 & 1.037646000 & -0.297666000 \\ 1 & 3.032850000 & -0.720568000 & 5.102138000 \\ 6 & -4.147842000 & 0.827762000 & -1.540528000 \\ 1 & 2.100047000 & 2.659278000 & 3.840850000 \\ 1 & -1.611275000 & 0.572530000 & -1.470752000 \\ 6 & 1.986411000 & 1.614130000 & 4.168826000 \\ 6 & -1.911712000 & -2.093197000 & 2.604144000 \\ 1 & 3.947056000 & 0.989233000 & 3.614030000 \\ 6 & -3.384693000 & -2.634740000 & 0.789950000 \\ 6 & -3.437120000 & 0.556052000 & -0.375434000 \\ 1 & -3.602920000 & 0.928802000 & -2.488048000 \\ 8 & 1.749578000 & -3.018945000 & 1.663796000 \\ 6 & -1.942416000 & 0.445129000 & -0.417415000 \\ 6 & -1.183021000 & -2.210721000 & -1.367453000 \\ 6 & -5.522053000 & 0.994716000 & -1.517674000 \\ 1 & -1.560405000 & 1.463661000 & 1.450280000 \\ 6 & -0.235609000 & -4.095763000 & -2.546422000 \\ 1 & -1.121409000 & -4.579797000 & -4.450213000 \\ 1 & 0.535471000 & 3.492038000 & 0.645291000 \\ 1 & 0.926686000 & 1.329475000 & 4.050446000 \\ 1 & 2.665152000 & 2.446294000 & 0.060739000 \\ 1 & 0.503061000 & -4.902393000 & -2.593667000 \\ 6 & -1.141113000 & -3.916514000 & -3.579502000 \\ 6 & -0.255368000 & -3.247653000 & -1.453162000 \\ 1 & 3.363740000 & -1.498913000 & 3.542857000 \\ 1 & -3.608287000 & 0.294383000 & 1.763737000 \\ 6 & -2.072520000 & -2.895100000 & -3.503994000 \\ 1 & -5.140837000 & -3.761042000 & 1.292980000 \\ 1 & 2.780946000 & 3.127555000 & 1.701681000 \\ 6 & -4.227230000 & -3.289440000 & 1.668917000 \\ 6 & 4.371935000 & 0.379216000 & 0.940330000 \\ 1 & 5.530380000 & 1.686577000 & 2.262954000 \\ 6 & 4.981076000 & -0.934865000 & 1.371677000 \\ 6 & 5.324669000 & 1.526589000 & 1.190337000 \\ 1 & 4.314968000 & -1.795618000 & 1.189448000 \\ 1 & 4.200563000 & 0.324025000 & -0.148111000 \\ 1 & 5.909127000 & -1.119792000 & 0.803850000 \\ 1 & 5.255298000 & -0.936461000 & 2.440919000 \\ 1 & 4.965435000 & 2.478795000 & 0.769037000 \\ 1 & 6.294694000 & 1.310375000 & 0.709083000 \\ 1 & 0.508359000 & 1.574884000 & -0.682112000 \\ 6 & -1.778380000 & 2.903221000 & -0.112873000\end{array}$




$\begin{array}{rrrr}6 & -2.636268000 & 3.665064000 & 0.667920000 \\ 6 & -1.434151000 & 3.358771000 & -1.383113000 \\ 6 & -3.148488000 & 4.862339000 & 0.194902000 \\ 6 & -1.940332000 & 4.559253000 & -1.851064000 \\ 6 & -2.801037000 & 5.312506000 & -1.067294000 \\ 1 & -2.914020000 & 3.308005000 & 1.667743000 \\ 1 & -0.739587000 & 2.772042000 & -2.002210000 \\ 1 & -3.826634000 & 5.450986000 & 0.821811000 \\ 1 & -1.657842000 & 4.913416000 & -2.848516000 \\ 1 & -3.202334000 & 6.259249000 & -1.443885000 \\ 1 & 0.288467000 & -0.228286000 & 2.161645000 \\ 1 & 1.214101000 & -0.291061000 & -1.322598000 \\ 6 & 1.528499000 & 0.359724000 & -2.249027000 \\ 6 & 3.024043000 & 0.072132000 & -2.351100000 \\ 6 & 3.540773000 & -1.208715000 & -2.182879000 \\ 6 & 3.902593000 & 1.117073000 & -2.599003000 \\ 6 & 4.904638000 & -1.439066000 & -2.242394000 \\ 1 & 2.854028000 & -2.042716000 & -1.970574000 \\ 6 & 5.270301000 & 0.893332000 & -2.660960000 \\ 1 & 3.479774000 & 2.121258000 & -2.715586000 \\ 6 & 5.776783000 & -0.384049000 & -2.477550000 \\ 1 & 5.297747000 & -2.451446000 & -2.092907000 \\ 1 & 5.954539000 & 1.729265000 & -2.848707000 \\ 1 & 6.856890000 & -0.562020000 & -2.517961000 \\ 6 & 0.816698000 & -0.289409000 & -3.437886000 \\ 8 & 1.223288000 & 1.622891000 & -2.099241000 \\ 1 & 0.973663000 & -1.379385000 & -3.529006000 \\ 1 & 1.180142000 & 0.186167000 & -4.366301000 \\ 1 & -0.267368000 & -0.096548000 & -3.375412000\end{array}$

Pro- $(R)$-ketone adduct with dihydride

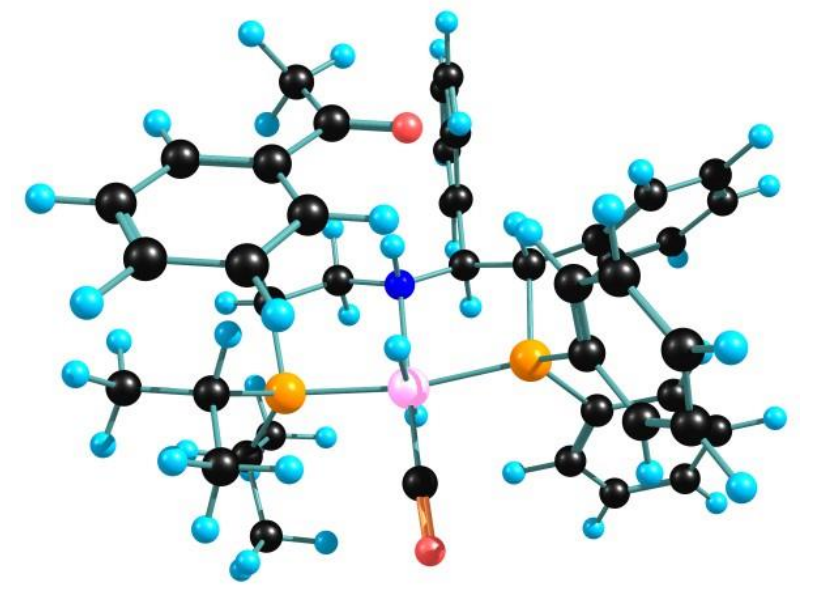

delta $\mathrm{H}$ (Hartrees) -2679.89 delta S (cal/mol/K) 288.334 delta G (kcal/mol) (323) -1681723.548
$1-5.347129000$
1.832862000
$-2.940436000$
$1-6.130685000$
1.357447000
1.258299000
11.772142000
$-3.184651000$
3.280849000 


$\begin{array}{lccc}6 & 2.832738000 & -2.907555000 & 3.413090000 \\ 6 & -1.095008000 & 1.284172000 & 1.025640000 \\ 15 & -1.333702000 & -1.098563000 & -0.230824000 \\ 6 & 2.536850000 & 1.064240000 & 1.904044000 \\ 1 & -2.231298000 & -3.544398000 & -1.570045000 \\ 1 & -0.356150000 & -0.569569000 & -5.058827000 \\ 6 & -4.095272000 & 1.081001000 & 0.634371000 \\ 7 & 0.322822000 & 0.916182000 & 0.958185000 \\ 1 & -4.105534000 & -1.497897000 & -1.238297000 \\ 6 & -3.853651000 & -3.153104000 & 2.244590000 \\ 1 & -1.791773000 & -2.523873000 & 2.239707000 \\ 6 & -5.891997000 & 1.619034000 & -0.866549000 \\ 6 & 0.802124000 & -2.837720000 & 0.608990000 \\ 1 & 1.251854000 & -0.838700000 & -0.630070000 \\ 1 & -0.425555000 & 0.196032000 & -2.737411000 \\ 6 & 3.003802000 & -1.404558000 & 3.363495000 \\ 6 & -2.810335000 & -1.930438000 & 0.442264000 \\ 6 & -0.838528000 & -0.790399000 & -2.979530000 \\ 6 & -5.052174000 & -3.168745000 & 1.553824000 \\ 6 & 1.102512000 & 1.502471000 & 2.038734000 \\ 1 & -3.778277000 & -3.628744000 & 3.228123000 \\ 1 & -5.932990000 & -3.652215000 & 1.989781000 \\ 6 & -5.433169000 & 1.355453000 & 0.414063000 \\ 26 & 0.609836000 & -1.114475000 & 0.768706000 \\ 1 & 2.479882000 & -1.205648000 & 5.441242000 \\ 15 & 2.570890000 & -0.749770000 & 1.688579000 \\ 1 & 0.661669000 & 1.146706000 & 2.987983000 \\ 1 & -6.951641000 & 1.833686000 & -1.039297000 \\ 1 & 3.152735000 & -3.299486000 & 4.393935000 \\ 6 & -3.658502000 & 1.349809000 & -1.701591000 \\ 1 & 2.347728000 & 0.320691000 & 4.552464000 \\ 1 & -1.184512000 & 1.118089000 & -1.090631000 \\ 6 & 2.192762000 & -0.767077000 & 4.469838000 \\ 6 & -2.740438000 & -2.538725000 & 1.691782000 \\ 1 & 4.069543000 & -1.157513000 & 3.540993000 \\ 6 & -4.023372000 & -1.963990000 & -0.249082000 \\ 6 & -3.188166000 & 1.068826000 & -0.422161000 \\ 1 & -2.952838000 & 1.353054000 & -2.542231000 \\ 8 & 0.895010000 & -3.980031000 & 0.496023000 \\ 6 & -1.752021000 & 0.714074000 & -0.228379000 \\ 6 & -1.385222000 & -1.598122000 & -1.982311000 \\ 6 & -4.998396000 & 1.616730000 & -1.925071000 \\ 1 & -1.489019000 & 0.772483000 & 1.927536000 \\ 6 & -1.745355000 & -3.316440000 & -3.644770000 \\ 1 & -1.179317000 & -2.837389000 & -5.667851000 \\ 1 & 1.032102000 & 2.608111000 & 2.037185000 \\ 1 & 1.113373000 & -0.949633000 & 4.335928000 \\ 1 & 2.972678000 & 1.513129000 & 0.988488000\end{array}$




\begin{tabular}{lrrr}
1 & -2.094636000 & -4.323643000 & -3.895243000 \\
6 & -1.233147000 & -2.491421000 & -4.630522000 \\
6 & -1.822046000 & -2.873965000 & -2.334099000 \\
1 & 3.413224000 & -3.441190000 & 2.644065000 \\
1 & -3.756524000 & 0.848088000 & 1.651218000 \\
6 & -0.776825000 & -1.227822000 & -4.291014000 \\
1 & -6.078389000 & -2.583620000 & -0.250384000 \\
1 & 3.144229000 & 1.431668000 & 2.748774000 \\
6 & -5.133774000 & -2.574205000 & 0.303309000 \\
6 & 4.093461000 & -0.963865000 & 0.663199000 \\
1 & 5.703933000 & -1.264366000 & 2.103024000 \\
6 & 4.226810000 & -2.332494000 & 0.037322000 \\
6 & 5.380889000 & -0.542628000 & 1.333140000 \\
1 & 3.325063000 & -2.620405000 & -0.526679000 \\
1 & 3.888182000 & -0.255094000 & -0.163766000 \\
1 & 5.072533000 & -2.340452000 & -0.672993000 \\
1 & 4.433681000 & -3.120207000 & 0.781249000 \\
1 & 5.315725000 & 0.450073000 & 1.810875000 \\
1 & 6.196206000 & -0.491291000 & 0.589610000 \\
1 & 0.690045000 & 1.299599000 & 0.080847000 \\
6 & -1.339035000 & 2.764707000 & 1.178135000 \\
6 & -1.826221000 & 3.268135000 & 2.377284000 \\
6 & -1.068127000 & 3.652541000 & 0.141416000 \\
6 & -2.037544000 & 4.626831000 & 2.544920000 \\
6 & -1.274041000 & 5.011259000 & 0.307860000 \\
6 & -1.759830000 & 5.503015000 & 1.509422000 \\
1 & -2.044890000 & 2.574103000 & 3.198895000 \\
1 & -0.685412000 & 3.280753000 & -0.818231000 \\
1 & -2.425127000 & 5.005212000 & 3.496627000 \\
1 & -1.060220000 & 5.698100000 & -0.518486000 \\
1 & -1.927867000 & 6.577444000 & 1.636498000 \\
1 & -0.018073000 & -1.244618000 & 2.190540000 \\
6 & 3.345719000 & 1.717509000 & -2.185454000 \\
6 & 4.627291000 & 2.045468000 & -1.744748000 \\
6 & 3.144533000 & 0.515320000 & -2.861839000 \\
6 & 2.171071000 & 2.589553000 & -1.938991000 \\
6 & 5.687736000 & 1.189861000 & -1.985638000 \\
1 & 4.808550000 & 2.982632000 & -1.207724000 \\
6 & 4.202007000 & -0.340004000 & -3.098031000 \\
1 & 2.130737000 & 0.258079000 & -3.187199000 \\
6 & 5.475691000 & -0.001869000 & -2.661813000 \\
1 & 6.692771000 & 1.455586000 & -1.642604000 \\
1 & 4.034214000 & -1.286395000 & -3.621649000 \\
1 & 6.315676000 & -0.679071000 & -2.851026000 \\
1 & 2.372164000 & 3.850604000 & -1.158962000 \\
& 1.076752000 & 2.278334000 & -2.350483000 \\
\hline & 1.437936000 & 4.425785000 & -1.130272000
\end{tabular}


$(R)$-transition state
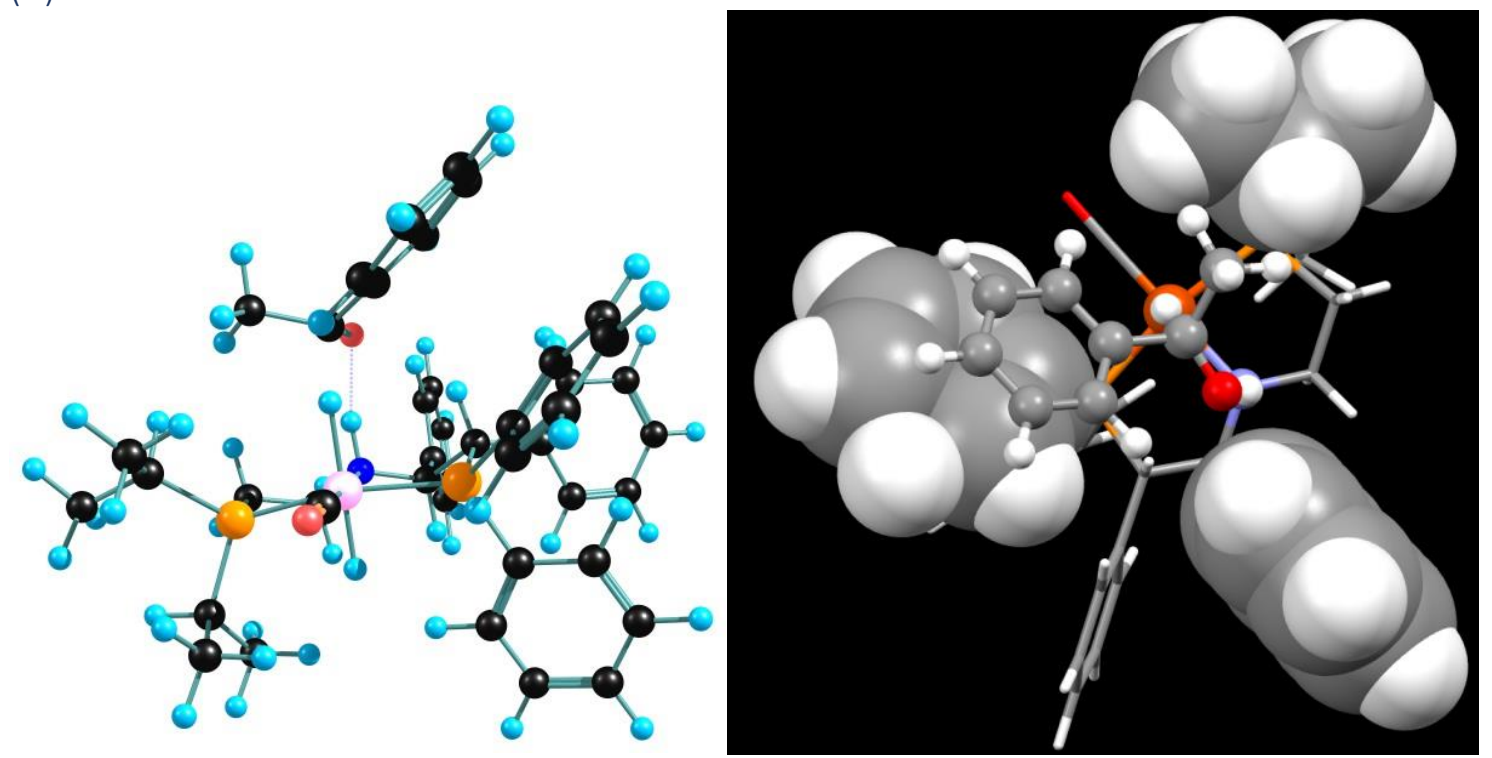

delta $\mathrm{H}$ (Hartrees)

$-2679.87$

delta S (cal/mol/K)

274.487

delta G (kcal/mol) (323) -1681705.751

$v$ imag $683.882 \mathrm{i} \mathrm{cm}{ }^{-1}$

$\begin{array}{lccc}1 & 5.801408000 & -0.000911000 & -2.003503000 \\ 1 & 5.305096000 & -2.560684000 & 1.411457000 \\ 1 & -3.505038000 & -0.540830000 & 3.160245000 \\ 6 & -4.463537000 & -0.896869000 & 2.744613000 \\ 6 & 0.652863000 & -1.810615000 & -0.468086000 \\ 15 & 0.636544000 & 0.427067000 & 0.976259000 \\ 6 & -3.018313000 & -2.250846000 & -1.029079000 \\ 1 & 0.023592000 & 2.563547000 & 2.710895000 \\ 1 & 3.514916000 & 4.299508000 & -0.341857000 \\ 6 & 3.522550000 & -1.641563000 & 0.647356000 \\ 7 & -0.743524000 & -1.435383000 & -0.724498000 \\ 1 & 3.045598000 & 1.167055000 & 2.505825000 \\ 6 & 1.455753000 & -1.863188000 & 4.290206000 \\ 1 & -0.108632000 & -1.744268000 & 2.822842000 \\ 6 & 5.712645000 & -1.291757000 & -0.280268000 \\ 6 & -2.138822000 & 1.309875000 & 1.453357000 \\ 1 & 2.691079000 & 2.006652000 & -0.626999000 \\ 6 & -4.238030000 & -1.828964000 & 1.574768000 \\ 6 & 1.380618000 & -0.217416000 & 2.520573000 \\ 6 & 2.273056000 & 2.624627000 & 0.171837000 \\ 6 & 2.638777000 & -1.322775000 & 4.763720000 \\ 6 & -1.577706000 & -2.629640000 & -0.804386000 \\ 1 & 1.002962000 & -2.723965000 & 4.793749000 \\ 1 & 3.129746000 & -1.753709000 & 5.642598000 \\ 6 & 4.884726000 & -1.886940000 & 0.657405000 \\ 26 & -1.484761000 & 0.017762000 & 0.473649000 \\ 1 & -4.022391000 & -3.532190000 & 2.862112000\end{array}$




$\begin{array}{lrrr}15 & -3.462885000 & -0.935579000 & 0.149829000 \\ 1 & -1.454037000 & -3.178875000 & 0.146995000 \\ 1 & 6.789712000 & -1.487923000 & -0.269270000 \\ 1 & -4.995756000 & -1.422677000 & 3.555859000 \\ 6 & 3.800562000 & -0.218964000 & -1.250229000 \\ 1 & -3.383253000 & -3.819348000 & 1.237134000 \\ 1 & 1.269804000 & 0.063259000 & -1.284244000 \\ 6 & -3.480095000 & -3.056806000 & 2.026377000 \\ 6 & 0.831121000 & -1.313617000 & 3.182355000 \\ 1 & -5.222408000 & -2.179995000 & 1.210050000 \\ 6 & 2.574538000 & 0.315980000 & 3.009689000 \\ 6 & 2.958286000 & -0.785369000 & -0.296912000 \\ 1 & 3.366518000 & 0.419195000 & -2.031579000 \\ 8 & -2.580267000 & 2.147759000 & 2.105702000 \\ 6 & 1.483760000 & -0.525376000 & -0.365618000 \\ 6 & 1.292189000 & 2.119411000 & 1.016264000 \\ 6 & 5.163149000 & -0.460465000 & -1.241610000 \\ 1 & 0.659803000 & -2.328128000 & 0.515280000 \\ 6 & 1.240153000 & 4.242675000 & 2.169689000 \\ 1 & 2.596727000 & 5.755481000 & 1.449870000 \\ 1 & -1.233634000 & -3.304345000 & -1.611383000 \\ 1 & -2.470170000 & -2.811059000 & 2.393947000 \\ 1 & -3.134464000 & -1.837931000 & -2.049459000 \\ 1 & 0.826796000 & 4.874822000 & 2.962476000 \\ 6 & 2.226012000 & 4.732538000 & 1.327421000 \\ 6 & 0.780707000 & 2.948055000 & 2.016475000 \\ 1 & -5.063721000 & -0.010152000 & 2.485081000 \\ 1 & 2.886898000 & -2.135345000 & 1.391705000 \\ 6 & 2.739435000 & 3.920187000 & 0.332283000 \\ 1 & 4.130560000 & 0.206530000 & 4.484023000 \\ 1 & -3.669985000 & -3.140795000 & -0.978390000 \\ 6 & 3.194256000 & -0.229215000 & 4.120055000 \\ 6 & -4.899976000 & -0.106412000 & -0.680384000 \\ 1 & -6.679315000 & -0.907321000 & 0.296898000 \\ 6 & -5.138588000 & 1.305294000 & -0.192732000 \\ 6 & -6.187422000 & -0.899460000 & -0.690507000 \\ 1 & -4.223776000 & 1.918915000 & -0.215658000 \\ 1 & -4.555669000 & -0.035133000 & -1.728419000 \\ 1 & -5.885947000 & 1.806633000 & -0.831569000 \\ 1 & -5.530900000 & 1.332674000 & 0.837611000 \\ 1 & -6.045932000 & -1.946980000 & -1.006006000 \\ 1 & -6.902835000 & -0.443017000 & -1.396030000 \\ 1 & -0.750015000 & -0.975947000 & -1.653693000 \\ 6 & 1.215918000 & -2.767739000 & -1.489056000 \\ 6 & 1.584556000 & -4.051768000 & -1.111655000 \\ 6 & 1.373591000 & -2.385191000 & -2.817864000 \\ 6 & 2.097549000 & -4.944555000 & -2.038575000 \\ 6 & 1.882288000 & -3.277928000 & -3.745395000\end{array}$




$\begin{array}{lrrr}6 & 2.246024000 & -4.558972000 & -3.359798000 \\ 1 & 1.465462000 & -4.357500000 & -0.064203000 \\ 1 & 1.087413000 & -1.373401000 & -3.131334000 \\ 1 & 2.384849000 & -5.953367000 & -1.723846000 \\ 1 & 2.001512000 & -2.966236000 & -4.788677000 \\ 1 & 2.651295000 & -5.260768000 & -4.096108000 \\ 1 & -1.476849000 & -0.939482000 & 1.648677000 \\ 1 & -1.460968000 & 0.947807000 & -0.889051000 \\ 6 & -0.405995000 & 2.551652000 & -2.239312000 \\ 6 & -0.882084000 & 3.584961000 & -1.439236000 \\ 6 & 0.722231000 & 2.770740000 & -3.020261000 \\ 6 & -0.251599000 & 4.815260000 & -1.431019000 \\ 6 & 1.347915000 & 4.007689000 & -3.024661000 \\ 6 & 0.858460000 & 5.034096000 & -2.235261000 \\ 1 & -1.751514000 & 3.409425000 & -0.791240000 \\ 1 & 1.086531000 & 1.950401000 & -3.649457000 \\ 1 & -0.626143000 & 5.616338000 & -0.784268000 \\ 1 & 2.227917000 & 4.173231000 & -3.656304000 \\ 1 & 1.353104000 & 6.011432000 & -2.231972000 \\ 6 & -1.137802000 & 1.244594000 & -2.423301000 \\ 6 & -2.560417000 & 1.443035000 & -2.898725000 \\ 1 & -3.054688000 & 0.467002000 & -3.003345000 \\ 1 & -2.512072000 & 1.894856000 & -3.906956000 \\ 1 & -3.167571000 & 2.106275000 & -2.265217000 \\ 8 & -0.510715000 & 0.277898000 & -2.899835000 \\ (R)-1 k 0 x 192014 & & \end{array}$

$(R)$-alkoxide adduct

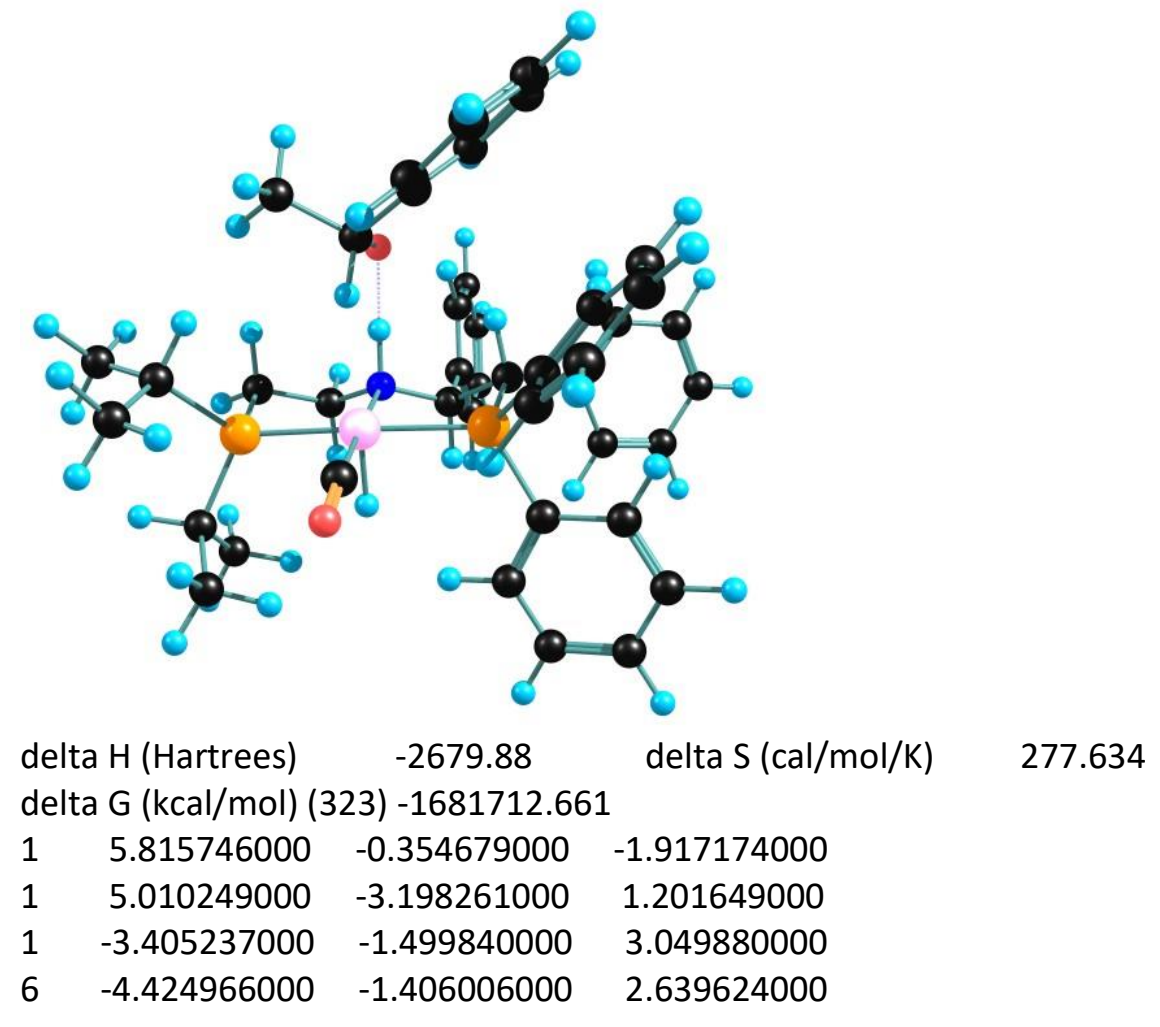




$\begin{array}{lccc}6 & 0.492709000 & -1.741247000 & -0.674392000 \\ 15 & 0.685597000 & 0.224785000 & 1.129784000 \\ 6 & -3.153874000 & -1.624194000 & -1.435374000 \\ 1 & 0.086241000 & 2.325255000 & 2.946560000 \\ 1 & 3.676293000 & 4.057414000 & 0.007087000 \\ 6 & 3.345552000 & -2.013929000 & 0.543356000 \\ 7 & -0.843386000 & -1.168401000 & -0.865881000 \\ 1 & 3.180725000 & 0.702427000 & 2.586994000 \\ 6 & 1.380970000 & -2.234715000 & 4.333589000 \\ 1 & -0.198354000 & -1.948813000 & 2.903113000 \\ 6 & 5.569406000 & -1.806523000 & -0.343977000 \\ 6 & -2.042757000 & 0.844014000 & 2.075157000 \\ 1 & 2.785103000 & 1.805966000 & -0.375613000 \\ 6 & -4.469637000 & -1.861359000 & 1.195490000 \\ 6 & 1.414071000 & -0.547740000 & 2.608482000 \\ 6 & 2.373989000 & 2.407096000 & 0.439424000 \\ 6 & 2.616844000 & -1.809125000 & 4.788862000 \\ 6 & -1.773898000 & -2.206561000 & -1.291692000 \\ 1 & 0.867894000 & -3.065049000 & 4.829873000 \\ 1 & 3.088467000 & -2.302942000 & 5.644825000 \\ 6 & 4.672725000 & -2.406260000 & 0.524940000 \\ 26 & -1.488190000 & -0.064480000 & 0.670439000 \\ 1 & -4.590264000 & -3.929043000 & 1.768374000 \\ 15 & -3.551052000 & -0.707031000 & 0.080540000 \\ 1 & -1.751047000 & -3.021883000 & -0.544287000 \\ 1 & 6.618870000 & -2.118015000 & -0.355222000 \\ 1 & -5.083414000 & -2.044179000 & 3.253247000 \\ 6 & 3.795085000 & -0.433018000 & -1.190197000 \\ 1 & -4.046423000 & -3.716625000 & 0.095522000 \\ 1 & 1.309721000 & 0.156917000 & -1.155606000 \\ 6 & -3.979843000 & -3.289564000 & 1.108126000 \\ 6 & 0.781300000 & -1.608529000 & 3.252793000 \\ 1 & -5.524068000 & -1.839447000 & 0.851413000 \\ 6 & 2.657358000 & -0.124599000 & 3.079880000 \\ 6 & 2.887934000 & -1.005602000 & -0.302895000 \\ 1 & 3.440556000 & 0.327734000 & -1.898474000 \\ 8 & -2.392995000 & 1.358276000 & 3.038695000 \\ 6 & 1.448917000 & -0.589474000 & -0.340978000 \\ 6 & 1.365958000 & 1.902348000 & 1.253678000 \\ 6 & 5.123401000 & -0.821735000 & -1.208970000 \\ 1 & 0.430469000 & -2.433505000 & 0.194010000 \\ 6 & 1.364710000 & 3.980787000 & 2.484488000 \\ 1 & 2.780118000 & 5.472785000 & 1.841739000 \\ 1 & -1.457324000 & -2.649757000 & -2.254795000 \\ 1 & -2.934616000 & -3.378887000 & 1.451122000 \\ 1 & -3.137518000 & -0.886711000 & -2.260620000 \\ 1 & 0.960037000 & 4.596382000 & 3.294465000 \\ 6 & 2.378969000 & 4.467583000 & 1.676054000\end{array}$




\begin{tabular}{lrrr}
6 & 0.865504000 & 2.708247000 & 2.276679000 \\
1 & -4.748165000 & -0.365125000 & 2.791154000 \\
1 & 2.652257000 & -2.510537000 & 1.232369000 \\
6 & 2.879515000 & 3.678572000 & 0.655516000 \\
1 & 4.229826000 & -0.406230000 & 4.512947000 \\
1 & -3.894842000 & -2.392568000 & -1.713616000 \\
6 & 3.252230000 & -0.750307000 & 4.159998000 \\
6 & -4.818192000 & 0.516385000 & -0.468167000 \\
1 & -6.462621000 & -0.850774000 & -0.908376000 \\
6 & -5.468341000 & 1.242535000 & 0.687516000 \\
6 & -5.863353000 & -0.062441000 & -1.395510000 \\
1 & -4.735488000 & 1.672778000 & 1.390489000 \\
1 & -4.224408000 & 1.259320000 & -1.035639000 \\
1 & -6.082254000 & 2.076912000 & 0.309304000 \\
1 & -6.143885000 & 0.584801000 & 1.260598000 \\
1 & -5.433499000 & -0.488656000 & -2.315413000 \\
1 & -6.569264000 & 0.727114000 & -1.705282000 \\
1 & -0.760948000 & -0.477269000 & -1.680813000 \\
6 & 0.996571000 & -2.534611000 & -1.854833000 \\
6 & 1.325510000 & -3.874057000 & -1.702036000 \\
6 & 1.157897000 & -1.936270000 & -3.101922000 \\
6 & 1.807983000 & -4.612687000 & -2.770503000 \\
6 & 1.634091000 & -2.676639000 & -4.170063000 \\
6 & 1.962357000 & -4.014429000 & -4.009094000 \\
1 & 1.202027000 & -4.348350000 & -0.719921000 \\
1 & 0.884897000 & -0.880462000 & -3.235540000 \\
1 & 2.064664000 & -5.668408000 & -2.632720000 \\
1 & 1.753170000 & -2.199473000 & -5.148987000 \\
1 & 2.341471000 & -4.594161000 & -4.857265000 \\
1 & -1.491920000 & -1.283035000 & 1.540155000 \\
1 & -1.457275000 & 1.497769000 & -1.061827000 \\
6 & -0.183231000 & 2.885080000 & -2.064690000 \\
6 & -0.443734000 & 3.987300000 & -1.256596000 \\
6 & 0.951755000 & 2.903340000 & -2.863612000 \\
6 & 0.393526000 & 5.088995000 & -1.263956000 \\
6 & 1.793330000 & 4.005810000 & -2.877662000 \\
6 & 1.511742000 & 5.106669000 & -2.085681000 \\
1 & -1.325669000 & 3.978975000 & -0.599849000 \\
1 & 1.148661000 & 2.022969000 & -3.486810000 \\
1 & 0.177275000 & 5.945939000 & -0.615295000 \\
1 & 2.680524000 & 4.009341000 & -3.522181000 \\
1 & 2.174603000 & 5.979018000 & -2.094316000 \\
6 & -1.140329000 & 1.700355000 & -2.169252000 \\
6 & -2.418724000 & 2.197621000 & -2.853457000 \\
1 & -3.137651000 & 1.367829000 & -2.965202000 \\
\hline & -2.167641000 & 2.546550000 & -3.871021000 \\
& -2.920458000 & 3.028351000 & -2.324494000 \\
\hline & -634921000 & 0.643274000 & -2.758006000
\end{tabular}

